\title{
New Game +
}

by

Nicholas Bava

A thesis submitted to the Faculty of Graduate and Postdoctoral Affairs

in partial fulfillment of the requirements for the degree of

Master of Architecture

in

Azrieli School of Architecture and Urbanism

\author{
Carleton University \\ Ottawa, Ontario \\ (C) 2021 \\ Nicholas Bava
}




\section{ABSTRACT}

This thesis connects architectural theories of virtual realities with the representation and experience of game space. It asks how architectural theory of the virtual can be better perceived and felt using the medium of video games, specifically representations of game space and the experience of playing within them. Game mechanics were understood along with a study of video game precedent to facilitate the creation of three game levels. During each level, and as part of the progress from one to another, the player can learn the mechanics of the game levels. Initial concepts of the levels experience are designed to change in the final level, challenging and illustrating how representation in the virtual context along with experience need not conform to notions of actual space. Even though the space is not actual, nor follows actual logic, it is still able to be perceived by the player as a reality. 


\section{ACKNOWLEDGMENTS}

Thank you to my parents Kim and Bruno, who took me in during this time of covid. The basement was freezing but it kept me alert and focused during my thesis year. Thank you to my bro Lucas who always keeps a smile on my face. I love you all.

I would like to acknowledge my inspirations. Those authors of visionary architecture books and those artists who I owe so much for inspiration and lessons. Thank you to Johan Voordouw without whom this project could not be completed. His critical eye and endless resources are as appreciated as they were taxing. Thank you to Neil Spiller who taught me to believe in my own capabilities without fear of the judgement of others. His lessons which emphasized the importance of creativity for both young and old architects will never be forgotten.

I would like to thank both Carleton University for the opportunity to complete my master's degree. I would also like to thank and acknowledge The University of Manitoba where I completed my undergraduate degree. The educational foundation that the school and proffesors provided me with were essential to my success. 


\section{TABLE OF CONTENTS}

ACKNOWLEDGMENTS

TABLE OF CONTENTS IV

LIST OF ILLUSTRATIONS V-VIII

TITLE PAgE 1

SECTION $1 \quad 2-23$

INTRODUCTION 4

Conceptual Frameworks: Representation, Experience, ANd Virtual Reality 5

ARCHITECTURE AND VIDEO GAME DESIGN 8

VIDEO GAME STUdIES 9

CASE STUdy GAMES 12

BEYOND CRIMSON StARS: A WALKTHROUgh 18

SECTION 2 24-45

THE GAME Body DIAGRAM 25

THE GAME SPACE DIAGRAM 28

MECHANICS OF DRAWING 31

CODING 32

UNITY GAME ENGINE 33

RESEARCH THROUGH DRAWING 34

RESEARCH THROUGH DRAWING: GAME MECHANICS 35

ReSEARCH Through DraWing: ModelLing ANd Unity SCRIPTS 42

SECTION $3 \quad 46-83$

DeVEloping THE GAME 47

OBJECT OF INTERACTION: THE SPHERE 48

LEVEL DESIGN AND EXPERIENCE 49

LEVEL 153

LEVEL $2 \quad 62$

LEVEL $3 \quad 72$

CONCLUSION 80

TERM DEFINITIONS 84

BIBLIOGRAPHY 87

TUTORIAL BIBLIOGRAPHY 88 


\section{LIST OF ILLUSTRATIONS}

fig.1 Counter Strike: Global Offensive "A" Site pg.3

fig.2 digital painting on 3d model of an imagined world with Mateo Linares pg.5

fig. 3 perspective from inside of a sphere displaced by a drawing pg.7

fig. 4 Study sketch of halo 2 landscape pg.9

fig. 5 Study sketch of Battlefield 3 pilot's view pg.10

fig.6 Fugue in Void in game screenshot Keever, Justin. Fugue in Void. October 11, 2018. Heterotopias. http://www.

heterotopiaszine.com/2018/10/11/brutal-interior-fugue-void/. pg.12

fig.7 Naissancee in game screenshot Naissancee. GiantBomb. Accessed May 28, 2021. https://giantbomb1.cbsistatic.com/

uploads/original/12/122165/2599871-9046638571-Naiss.jpg. pg.13

fig. 8 Manifold Garden in game screenshot Manifold Garden. September 15, 2015. Flickr. https://www.flickr.com/photos/

playstationblog/21449477405/in/dateposted/.pg. 14

fig.9 Beyond Crimson Stars in game screenshot pg.15

fig.10 Inside in game screenshot Inside. July 7, 2016. Imugr. https://imgur.com/XSIU95D?nc=1. pg. 16

fig.11 Beyond Crimson Stars in game screenshot pg.17

fig.12 Beyond Crimson Stars entrance to the mall screenshot pg. 18

fig.13 Beyond Crimson Stars chrome tombstones screenshot pg. 19

fig.14 Beyond Crimson Stars bottle of bleach screenshot pg. 20

fig.15 Beyond Crimson Stars school of bowling pins screenshot pg. 21

fig.16 Beyond Crimson Stars house of memories screenshot pg. 22

fig.17 Beyond Crimson Stars house of memories screenshot pg. 23

fig.18 game body diagram pg. 26 


\section{LIST OF ILLUSTRATIONS CONTINUED}

fig.19 game body diagram details pg. 27

fig.20 game body diagram details pg. 27

fig. 21 game space diagram pg. 29

fig. 22 game space diagram details pg. 30

fig. 23 game space diagram details pg. 30

fig. 24 game space diagram details pg. 30

fig. 25 hybrid drawing of level 2 consisting of hand drawing, rhino, unity, and photoshop pg. 31

fig.26 Unity Game Engine General User Interface pg. 33

Fig. 27 Hand-drawn Base Maps Pencil drawings stretched over basic mesh objects pg. 36

Fig.28 Random Placement of Objects C\# scripts instantiate objects textured with hand drawings placeing them within boundaries pg. 37

Fig.29 Procedural Scale, Rotation, Material Selections C\# scripts rotate, scale, and select drawings to be placed on planes and

instantiated into the gamespace pg. 38

Fig.30 Height Maps, Vertex Displacement, Particle Systems The surrounding 3-dimensional geometry starts as a flat surface, where

the pencil marks are darkest the mesh objects add depth. A particle system creates and destroys mesh objects textured with pencil drawings pg. 39

Fig.31 Pixel Displacement and Field of View (FOV) The pencil markings sit on a 2-dimensional plane but are rendered by the engine as having depth. The fi eld of view of the character camera distorts and alters the world around an aspherical fisheye lens pg. 40

Fig.32 Physics and Force Based interaction with objects, Mesh Displacements, Directional Movement Forced based interactions and mesh displacements cause the textured planes to be forced away from each other. The planes fall under the engines simulated gravity and the character is able to move and observe these planes pg. 32

fig. 33 Perspective and elevation collage pg. 43 


\section{LIST OF ILLUSTRATIONS CONTINUED}

fig. 34 Perspective and section collage pg. 44

fig. 35 Perspective and plan collage pg. 45

fig. 36 Design sketch on yellow pad pg. 47

fig. 37 level 1 Interactable Sphere pg. 48

fig. 38 level 2 Interactable Sphere pg. 48

fig. 39 level 3 Interactable Sphere pg. 48

fig. 40 Early concept drawing for the level 3 environment with adjacent detail pg. 50

fig. 41 Early concept drawing for the level 3 environment with adjacent detail pg. 51

fig.42 Plan-square platform enclosed on all sides with a cauldron in the center atop a plane pg. 54

fig.43 Plan- The light from the centre of the cauldron obstructed by collection of 2-dimensional planes pg. 54

fig. 44 Section-Collection of hand drawings textured on 2-dimensional planes dormant above the cauldron with the player and the sphere currently unaffected by gravity pg. 55

fig. 45 Sections- Series of sections as the player interacts with the different spheres, causing deformations and glitches in the collection of drawings. Boids can be seen flying. pg. 55

fig. 46 Object-2-dimensional planes are subject to the effects of playing with the spheres pg. 56

fig. 47 Object- twists and glitches as the spheres are thrown by the player pg. 57

fig. 48 Al boids-Simple geometries or pyramids in this case are the standard form that boids take when being developed pg. 57

fig. 49 Al boids- Based on the movement of schools of fish or flocks of birds the boids move, avoid, follow, and feed on virtual

objects pg. 58

fig. 50 2D drawing as 3D space- A 2-dimensional plane is textured with an ink sketch pg. 58

fig. 51 2D drawing as 3D space- Pixel displacement renders black strokes as depth pg. 60 
fig. 52 Level 1 glitch collage pg. 63

fig. 53 Plan- A recreation of Hang em High, a map from Halo CE pg. 63

fig. 54 Plan- The players goal is to bring the sphere to the now much smaller cauldron on the opposite side of the map pg. 64

fig. 55 Elevation- Undisturbed original map before the game has begun pg. 64

fig. 56 Elevations- As ball moves towards the objective 3D geometries begin to deform and glitch, hindering the player and changing the layout of the level. These geometries are the result of play within the games configuration. pg. 64

fig. 57 Axonometric and Perspective Views during Gameplay- The geometries take new shapes and "break" their standard mesh material renderings. pg. 65

fig. 58 New Level Painting- Upon completion, the level could generate a new mesh based on the movements from the player. A new layout would be generated based on the representation of 3D space that was left behind by the player resulting in a new experience with detail above pg. 66

fig. 59 Plan- Possible new generation based on deformations during play pg. 67

fig. 60 Axonometric pg. 67

fig. 61 Perspective- Playing the new map pg. 68

fig. 62 Digital painting of continued generation pg. 70

fig. 63 Perspective- The level offers a large mountainous area to be explored with diffrerent spatial and material experiences pg. 73

fig. 64 Perspective- The concrete tiles begin to bend and move as the player approaches the bridges from level 2 now embedded in the snow pg. 73

fig. 65 Perspective- Level 2 pg. 74

fig. 66 Perspective- Level 2 acting as a boid, at a new scale in Level 3 pg. 74

fig. 67 Perspective- A pool of water has been replaced with wood pg. 75

fig. 68 Perspective- As the player walks past they causes the pool of wood to ripple and wave pg. 75

fig. 69 Perspective- Large planes, rocks, and doors have been textured with a live view of the player's perspective pg. 76

fig. 70 Perspective- As the player approaches the panels distort, extend to infinity, or show their plans and elevations pg. 76

fig. 71 Perspective- The original map from Level 2 floats along side the 2-dimensional planes from Level 1 pg. 77

fig. 72 Perspective- The planes orbit in a circular pattern while some of level 2's map reflects the sky while other areas reflect the players gaze pg. 77

fig. 73 Level 3 perspective and glitch collage pg. 79

fig. 74 Performance triptych pg. 80

fig. 75 Performance triptych detail pg. 81

fig. 76 Performance triptych detail pg. 82 


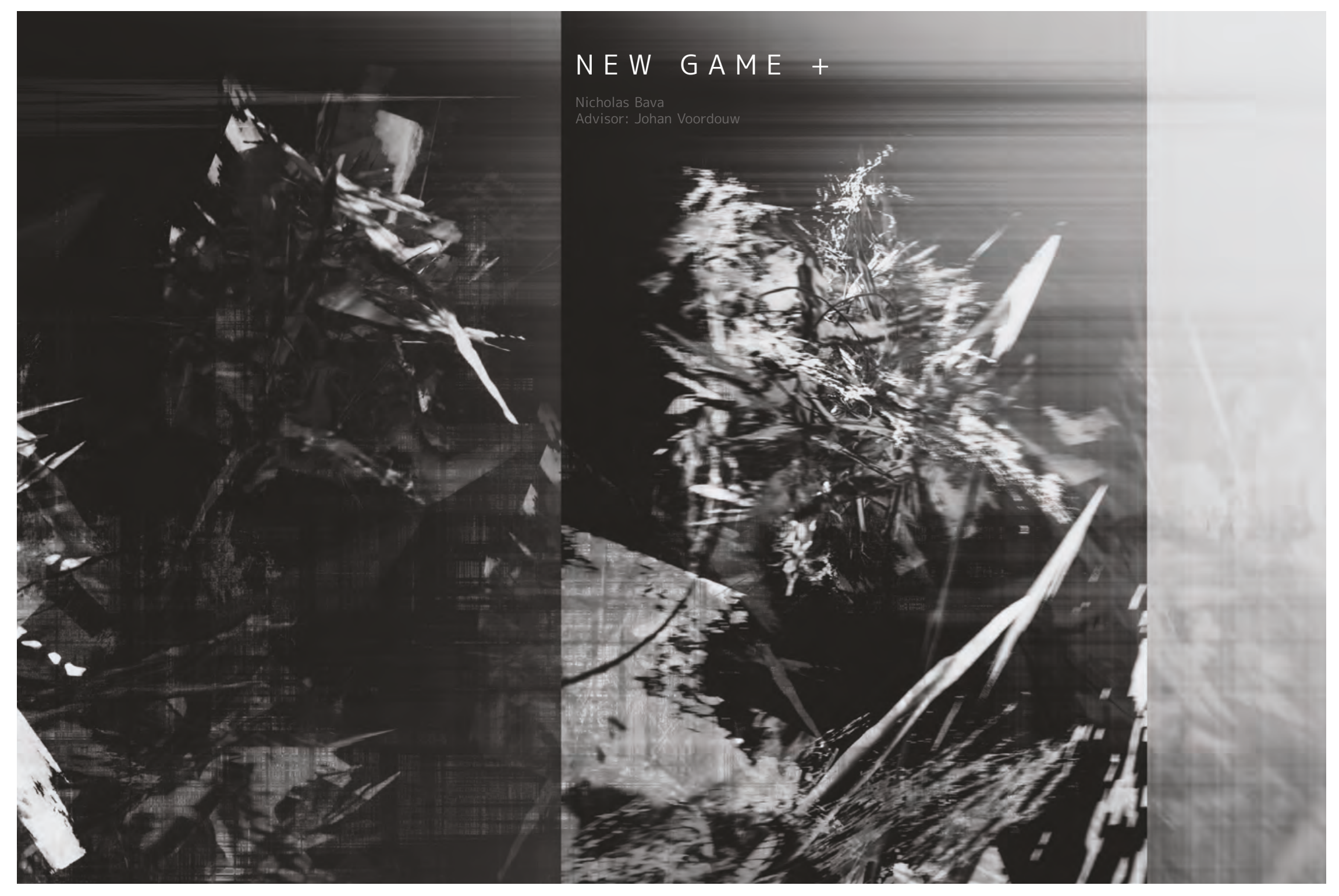




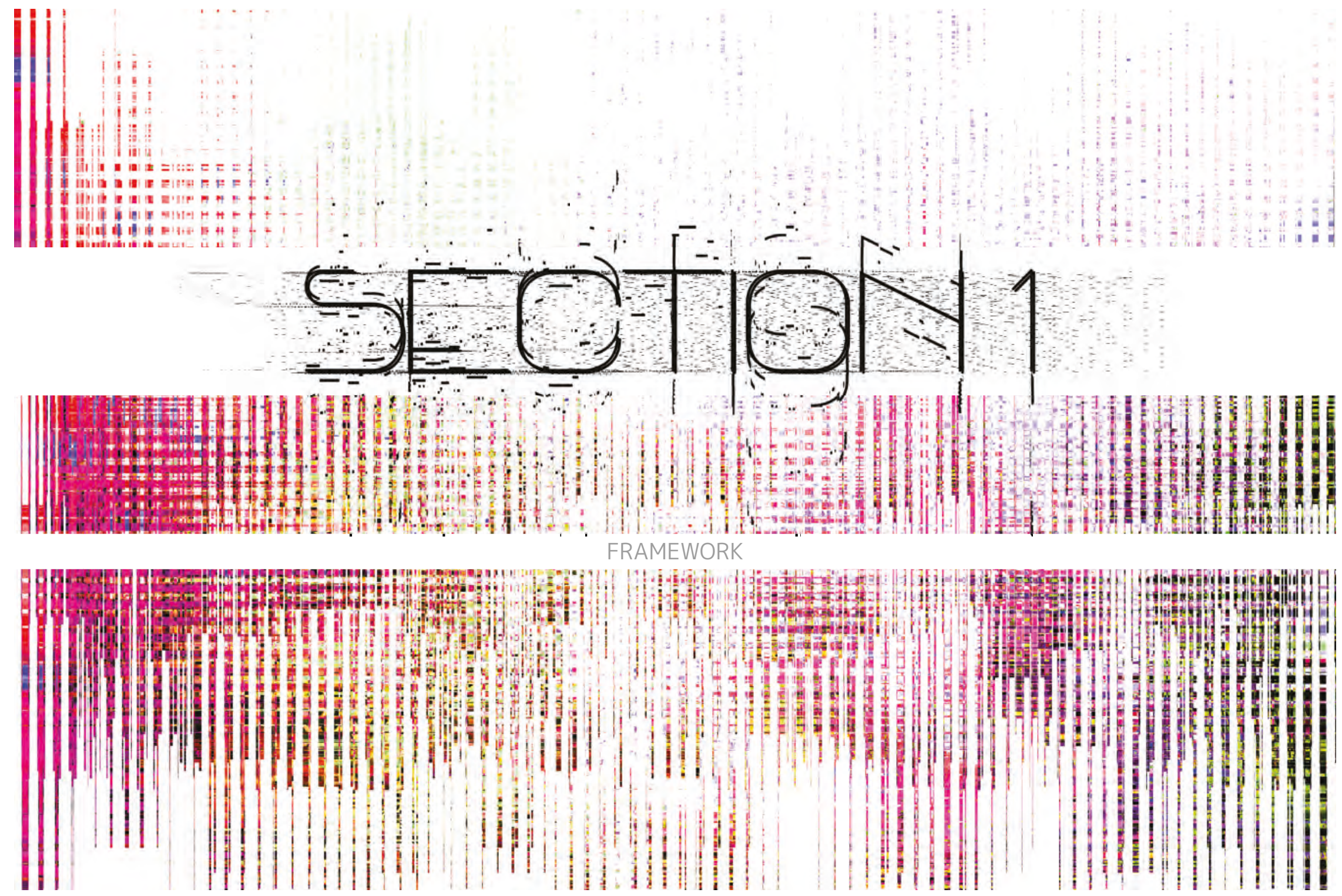




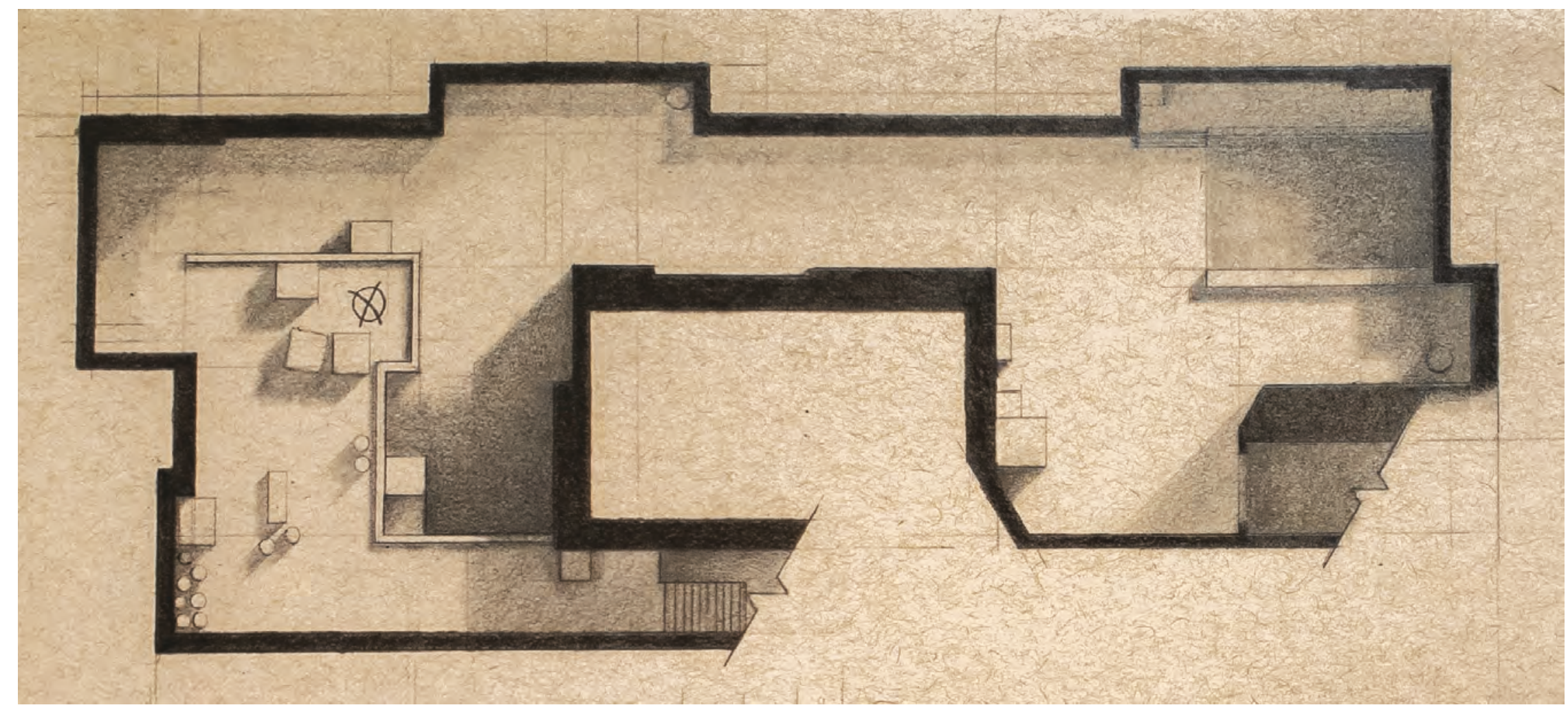

fig.1 Counter Strike: Global Offensive "A" Site 


\section{INTRODUCTION}

Reading books opens the mind to imagined worlds, movies bring those worlds to life on the screen, video games allow you to experience imagined worlds. As I reflect on my early childhood, I can remember the influences these works left on me. Science fiction was and still is my favourite genre of storytelling. I cannot forget Larry Niven's Ringworlds, Orson Scott Card's zero gravity war games, Isaac Asimov's tales of Foundation, and Frank Herbert's sand worms displacing the dunes as they travelled across the desert. Video games left a different kind of impression on me. I remember being part of great battles on Halo's ringworlds, flying a helicopter as 200 people worked together in Battlefield 3, exploring the fallen utopia of Bioshock's underwater city, and selling my art on the sides of cars in Forza 3. Millions of other people shared time in these worlds with me, making their own choices, affecting my own. My favourite novels are special to me because they are my imagined worlds. Video games are special to me because of how I affected their worlds while sharing in that experience with so many others.

A game's world is the space that a player moves his character through while playing that game. Game worlds can be divided into two categories, single player levels and multiplayer maps. Fundamentally a level and a map are the same thing, a virtual environment where gameplay takes place. Levels are defined by their connection to a narrative. Skipping from level 1 to level 5 means that a chunk of the story has been lost, like skipping 4 chapters of a book. A map is a self-contained space that does not need a connection to the next map often as part of the multiplayer section of a game. Dust 2 is a map that was developed in the late 1990's for the game Counter Strike. Considered a classic, this map has been continually updated and is played competitively to this date. I can close my eyes and walk around that map like any other actual world space. According to game statistics I have spent 53.5 hours playing on Dust 2 . With experience I know that if I plant the bomb on the corner of 'A Site', I can retreat down to the ramp known as Pit and easily eliminate the enemies as their heads show above the guard rail when they come to defuse the bomb. Equally experienced teams know that when the bomb has been planted in that corner deploying a smoke screen for cover will easily allow them to win the round. I have spent more time running around this map than any architectural project I have brought from concept to completion. For me, this map is as real as any other space that I have visited. For this thesis I am designing my own game with its own levels with the intention that others and myself can play them. 
Conceptual Frameworks: Representation, Experience, and Virtual Reality

Representation and experience are two concepts that have a theoretical history in the field of architecture. Architectural representations allow the architect's intentions to be translated to built form. The traditional medium of an architectural representation is the drawing. Swati Chattopadhyay expands the definition of architectural representation in the article "Architectural Representations, Changing Technologies, and Conceptual Extensions". She includes drawings and virtual models as projections that go beyond actual construction saying, "they extend and elaborate the fabric of buildings in dimensions and locations that cannot be realized in lived tactile space" (Chattopadhyay 2012, 271). A variety of artistic mediums have been used for architectural drawing. A technological evolution has occurred from chalk on paper to the pixel approximation of the vector line on a screen. The drawing communicates the perfect form of the building. A reality where any misinterpretation or human error during construction does not exist. If the plan on a set of construction documents made from a digital model can be considered a drawing, then the same plan drawing of a game's level is both a representative drawing and the thing. The built form of architecture and its representations often exist in separate realities. The actual building does not exist on paper or within modelling software. Video game design does not have this same disconnect. A game's space can be designed, modelled, and played all within one reality. If a game's representation and playable space exist in the same reality, then the representation can be experienced through playing in that space.

FIG.2 DIGITAL PAINTING ON 3D MODEL OF AN IMAGINED WORLD WITH MATEO LINARES

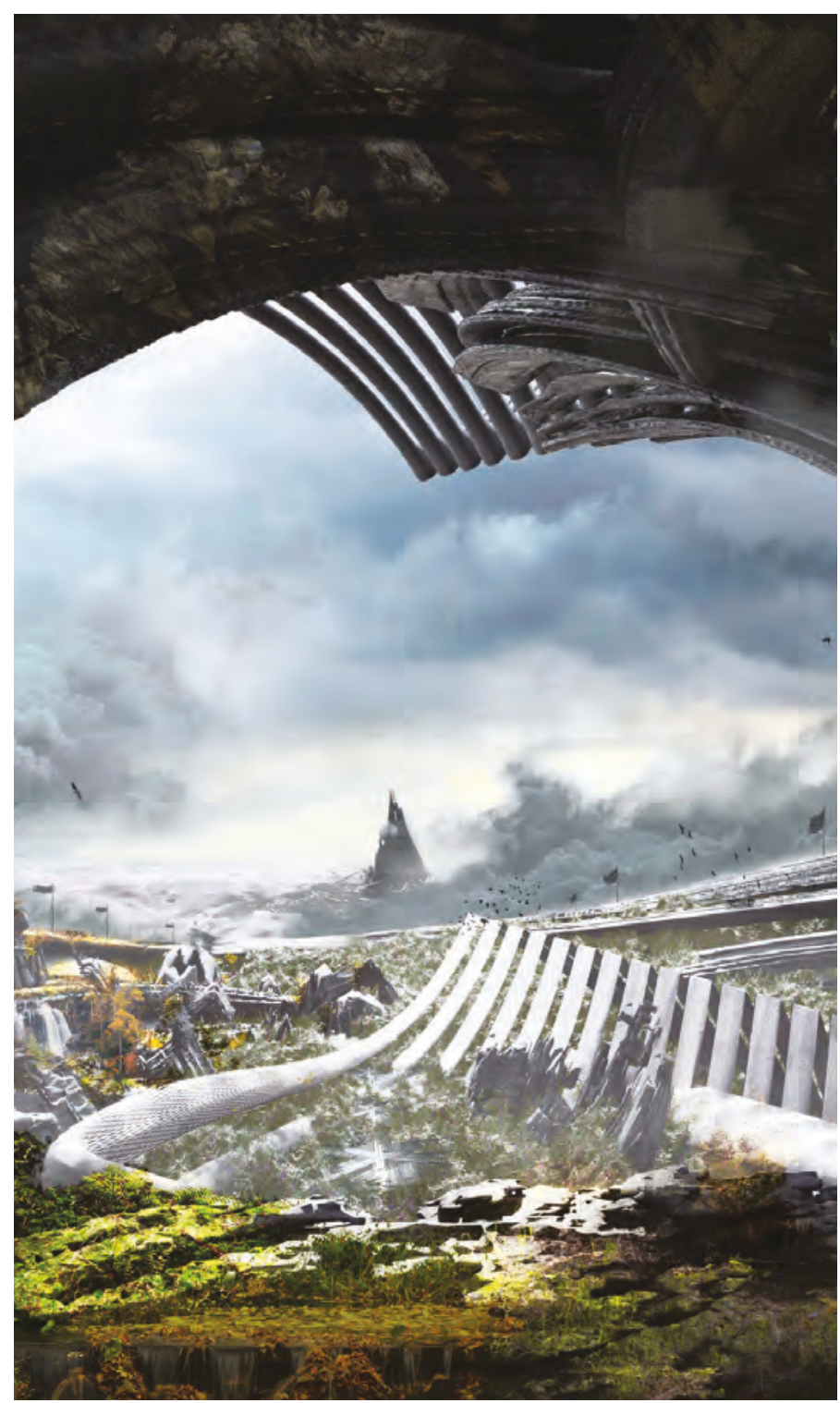


A video game space and an architectural space can both be experienced by an individual. Chattopadhyay further elaborates that "virtual space as prosthetics seeks to extend our sensory reach and minimize the distinction between virtual and real space in immersive digital environments" (Chattopadhyay 2012, 271). This thesis is not concerned with the idea of prosthetics that enhance interaction with the virtual, such as headsets or haptic controllers. A video games ability to minimize the boundaries between real and actual are a key part of the experience of virtual space. When the body experiences a space in the actual world all available senses are actively engaged. Sitting in a packed restaurant the background noise of the guests can mask the waiter's footsteps as they drop off a plate of fresh pasta. The smell of fresh tomato mixed with basil hits your nose before you bring the cold silverware to your tongue. The heat of the candle mixed with the taste of fresh vegetables brings you back to a vacation on the coast. The senses engaged when dining in a restaurant do not all translate to the same scenario within video game. You are no longer within your own body, but you are seeing through the eyes of a playable character. Our sight translates almost seamlessly between the two realities, the same with sound. The senses of taste and smell are completely absent when we play the scenario. Touch or a sense of feel is translated through our body's inputs with the game. A game can "feel" unresponsive or slow, or it can feel tight and fast. This "feel", or experience is controlled by the code that makes up a game. The representation of a game and its experience are both within a virtual reality. A game exists as a virtual reality that structurally, is made of code. A game is perceived as code rendered on a screen, but the experience of the represented space is real.

Contemporary use of the term virtual reality has become diluted across industries and popular media. This thesis defines virtual reality based on architectural theory in relation to digital representation. Architecture, like video games, is rooted in virtual realities. Antoine Picon in his paper "Architecture, Science, Technology, and the Virtual Realm" defines the fundamental feature of virtual realities as a reality, but a potential one (Picon 2003, 295). He emphasizes design as one of the virtual dimensions of architecture (296). Video game design and contemporary architectural design share the use of virtual realities in order 


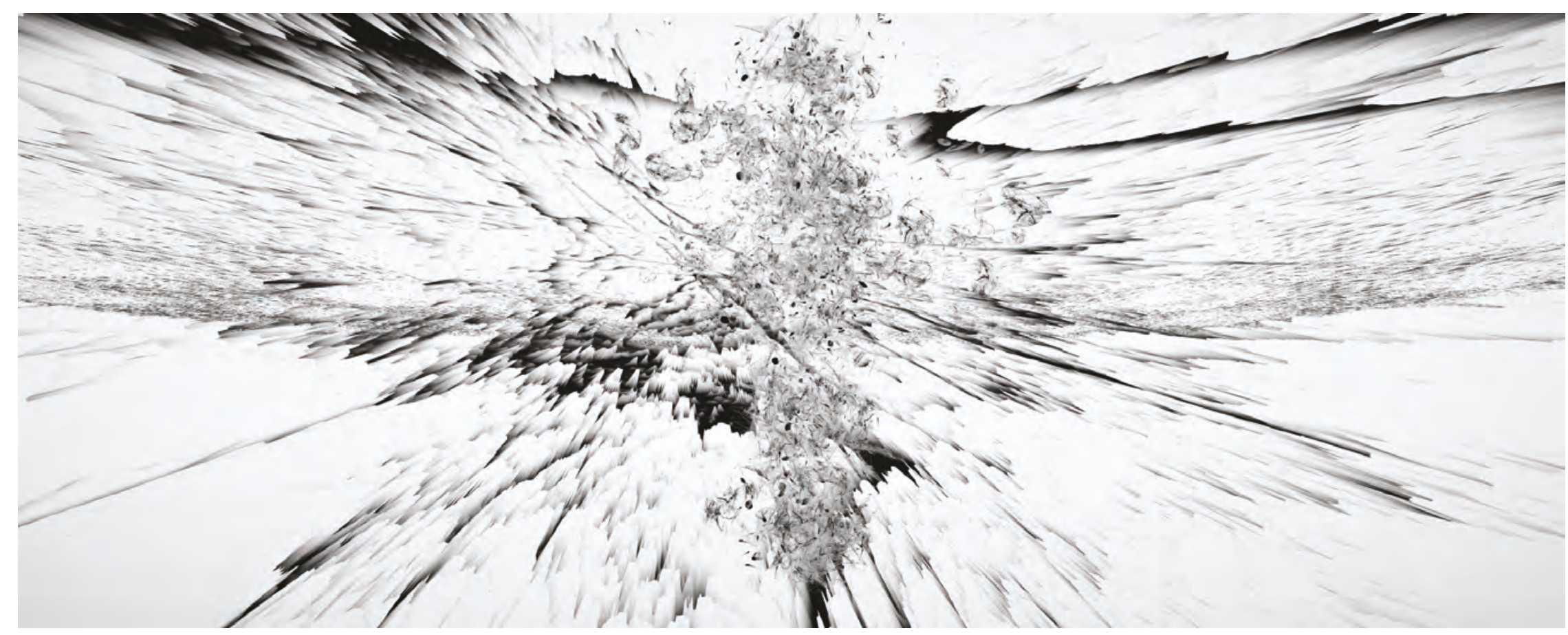

to express the designer's intentions. Drawing and representation are both virtuality's of design. Grosz in her section on Cyberspace, Virtuality, and the Real, posits that virtual realities have been present with us since the idea of the trace (writing and culture). She expands this idea further by saying "virtual reality of computer space is fundamentally no different from the virtual reality of writing, reading, drawing, or even thinking" (Grosz 2001, 78-79). When text is read from actual paper it is comprehended virtually, video games are also comprehended this way, but they exist only virtually. Virtual realities in architecture began on paper where (architectural) representation was used to convey meaning behind drawings. Contemporary practice utilizes virtual realities in the same way, except the drawings are now constructed digitally. Virtual reality in architecture has the potential to go beyond approximations of the actual world, becoming spaces of their own. Grosz describes the potential of the virtual that this thesis aims to explore. She says, "the virtual is the space of emergence of the new, the unthought, the unrealized" (78). Video game design offers a way to interact with virtual reality that is affective - where the viewer becomes a player in these spaces that can represent impossible experience. 


\section{Architecture and Video Game Design}

Late nights, unreasonable deadlines, low wages, professional game design and architecture share all their most glamorous hallmarks. Architectural design has evolved to fully embrace digital design techniques. Computer aided design has become a permanent facet of contemporary architectural theory and design. Video game design has evolved in tandem with the development of computers. Architecture as a field has divided itself from the construction of buildings to focus on design. If the contemporary architect now focuses on communicating the drawing of a digital design to be built, the game designer undertakes the designing and building. It is more accurate to say that building and designing in the virtual environment are the same thing. The architect builds the digital model of a home and then represents that on the drawing sheet, the game designer builds the digital model of a home and moves in.

Game design and architectural design begin to diverge once the digital model has been completed. From this point an architect will begin to detail connections, represent wall constructions, and specify each element of the design, including everything a builder would need to construct the actual building. An architect would then oversee the construction of the building. From the game designers' point of view, the building has already been constructed. Instead of observing a contractor mortar joints between bricks texture artists create the joints from an image. If necessary, the game designer can now place the building within the virtual environment of the map.

Architects design with the human body in mind. Games are designed with the player character in mind. The player character may not look or move like an actual human (or even be human) but all the spaces are designed around who or what the player is controlling. The result is spaces designed in games can be specific to the one or two player characters that everyone is able to access. Architecture tends towards general standards for heights, widths, openings, etc. Hyper specific design down to the length of a pixel can be found in abundance in games. Dust 2 would be played differently if the guardrail were raised a couple inches concealing player movements around the 'A Site' objective. Both forms of design take into consideration the bodies that use a space- a game has the unique opportunity to design what body will use a space. 


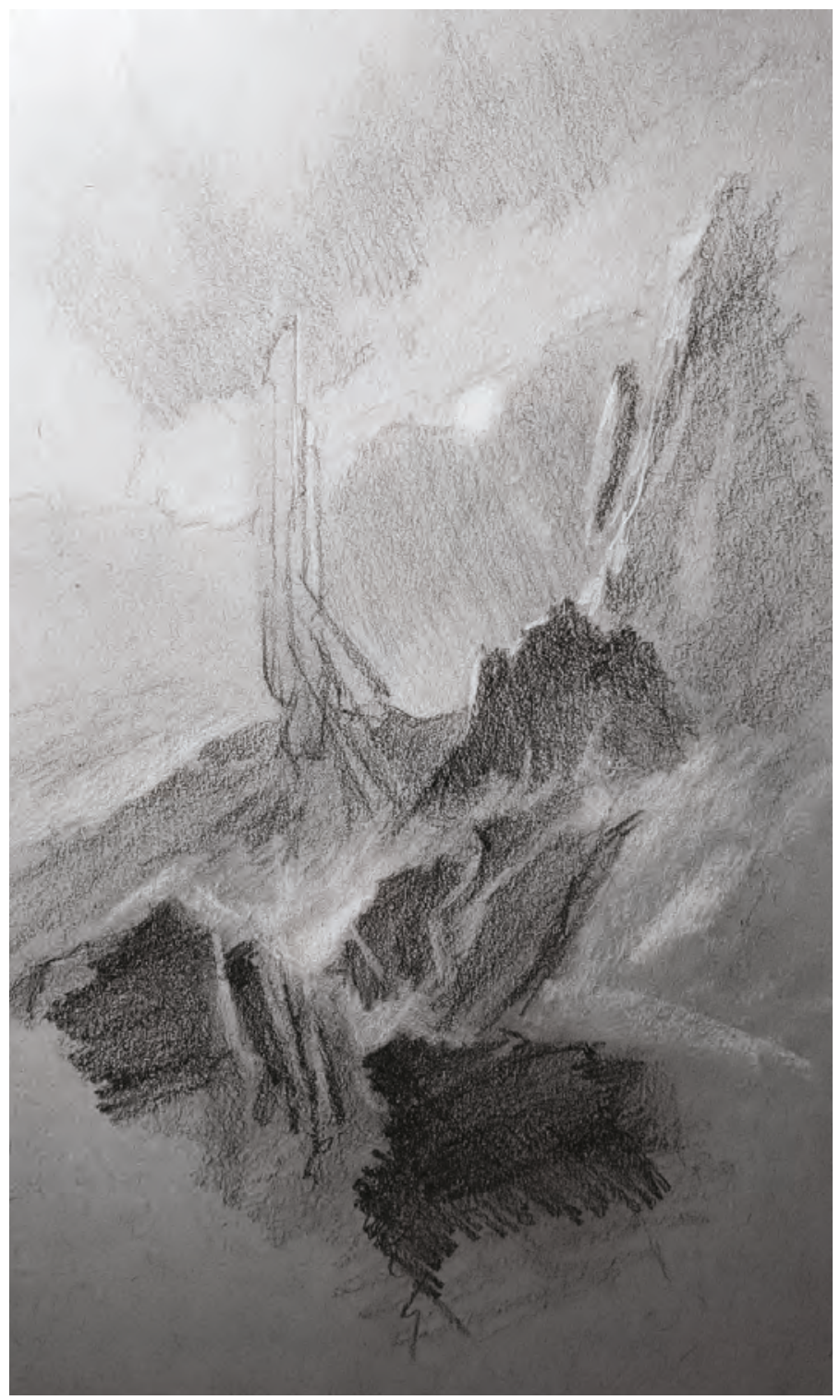

Video Game Studies

The context of a video game deals with the unique themes of play and its relation to how it feels. The research conducted into video games, as well as media studies focused on ideas of representation and experience aiming to connect them to architectural theory. Experiencing a video game is colloquially described as 'playing' a video game. The term play in video games reveals one of the unique qualities of the video game experience, the ability to succeed, but more often the ability to fail. Aubrey Anable in her book Playing with Feelings: Video games and Affect defines play in video games. With the repeated failures comes the development of skill. The player's reward for their perseverance is the chance at completing the game without error. This process is described as videogames transporting "our digital failures into the realm of play" (Anable 2018, 103) These repetitions can interestingly involve death or the destruction of virtual spaces impossible in the actual world, but in this continual play, in this succession of success and failure the operations that govern play are experienced, and its spatial representation learned.

FIG.4 STUDY SKETCH OF HALO 2 LANDSCAPE 


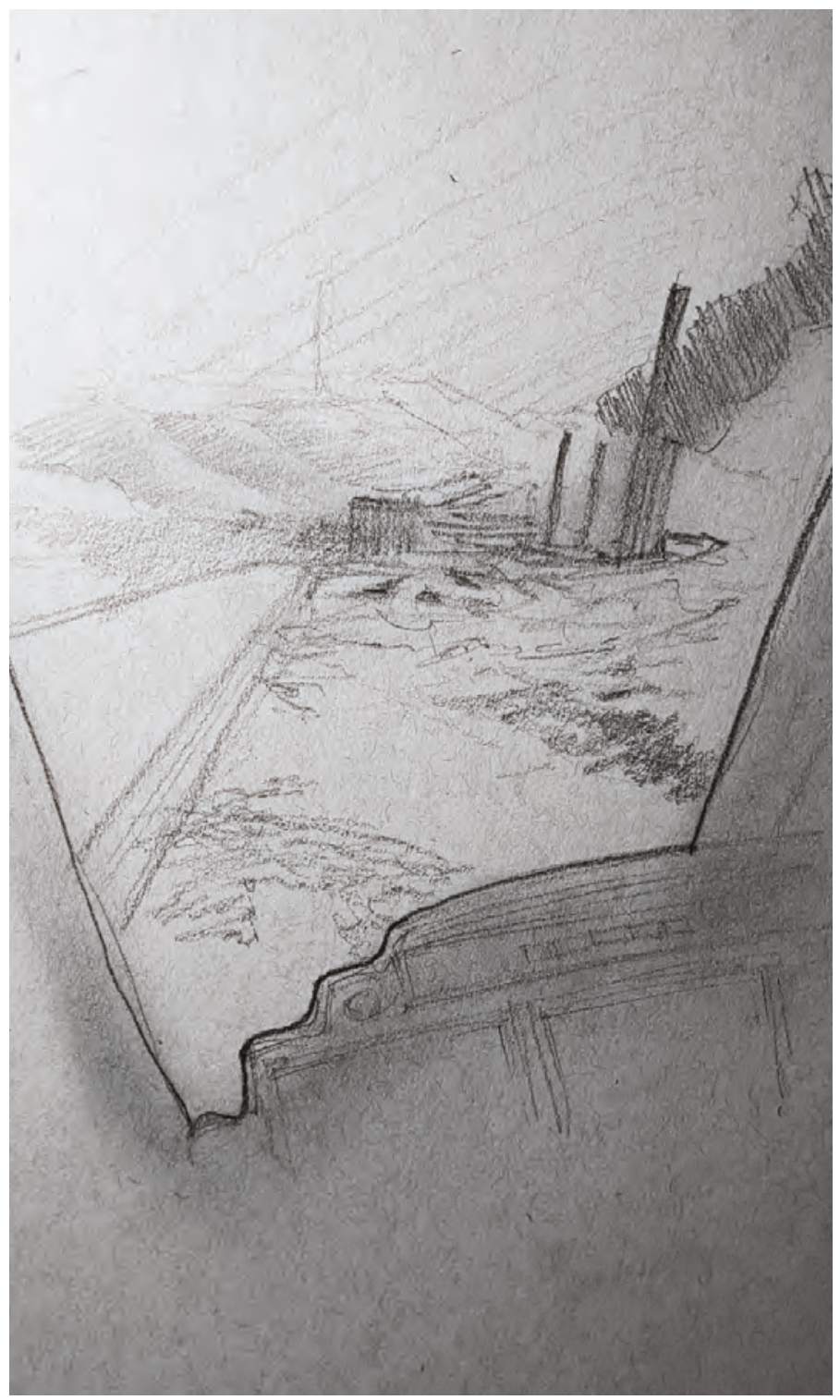

A game must be played to be experienced. Having established that play is the way that we interact with a game, what exactly makes up the rules of this experience. Code is the architecture that makes up a game and its gameplay. Anable writes that games: "are also part of a process of training us how to feel about these devices and the larger systems for which they are proxies." (128) Following this thought it could be reasonably assumed that understanding the code could also allow us to understand the experience of a video game. However, as Anable illustrates, the experience and the code that defines their rules are not interchangeable. The experience of a video game lies in the choices the player has in relation to the rules that the game has defined, or the 'configuration' (52). The experience is how we choose to act within the game, she summarizes the idea by writing "Video games compel us to act and be acted upon through the procedures of their algorithmic structures, through the way these structures are given representation form visually, narratively, and aurally, and through the designed intimacy of their interfaces and the contexts of our play." (52) A games experience can not be described simply by its code the same way architectural experience can not be described simply by how its structure acts against gravity. It is necessary to allow players to play with the rules, be it follow them or break them (a glitch), in order to allow them to experience the game spaces reality.

FIG.5 STUdY SKETCH OF BATTLEFIELD 3 PILOT'S VIEW 
To act and to be acted upon within a game space requires a player on one side of the screen and a character on the other. It is through our bodies input and feedback with technology that we can 'feel' what a game is like. Research Fellow Brendan Keogh at Queensland University discusses in his book $A$ Play of Bodies: How we Perceive Videogames what it means to "feel" a video game and by extension, a virtual reality. The "play of bodies" that occurs between the player and the player character is what constitutes a game's "feel". If we consider a game like Flower an experience where we forgo a body and control the wind carrying petals over rolling landscapes. What does it mean to say "the minimalist experience of Flower feels relaxing? How does a video game "feel" like anything at all?" (Keogh 2018, 21) The feel of the game comes from the players actions in the context of the game's rules. The feel is closely connected to the games code which has the most direct impact on feedback to a player's controls. The code here is mediating the communication between the players body and the characters 'body' whatever form that may take. For the thesis games levels to communicate an experience the development of the games code will need to consider how it might 'feel' to play a game. This is the closest control the designer has over how players body will interact with the body in the virtual environment, dictating the tone of the experience.

As I consider what experience I want the player to have in my own game and space I must consider theories of representation and experience. It will be necessary to code in a way that at times it can be broken in order to illustrate the structure of the virtual. I will not limit the players interaction with elements of the games space. If an object can be deformed it will be allowed to do so without limit. I will design the representations of objects in my game to both allude to their virtual environment and contradict their actual world counterparts. This juxtaposition can be used in order have the player consider the rules of the reality they are playing within. They are already examples of published games that have explored these concepts in various ways. I have chosen some precedent games to study and play in order to learn strategies for representing within virtual realities. They will also help me to critically evaluate how I experience games and how I can convey these experiences to the reader. 
Case Study Games

To develop the game for this thesis, I researched five video games as a series of case studies. In the following section, I describe representational and experiential qualities of each game in order to catalogue how they tackled representation and experience within their contexts.

Fugue in Void is an example of how atmosphere can create immersion in a digital world. A bridge fades away into a dark fog as the game begins. The crashing of stormy waters at the base of the bridge invites the player to look over the edge. Black water below and a dark fog ahead pull the player forward. A structure stretches across what might be a coast with two stairs welcoming the player inside. The large structure is filled with wet black bio-mechanical tubes descending into a hole. The only way to progress is to drop down with the pipes. The games opening moments use fog effectively to hide to what might lie before the player. The waters audio works well to differentiate the players transition from exterior to interior. Only having one route forward means that the player must confront the imagery of the bio-mechanical parts entering the stone structure and reconcile why it might be there in their mind.

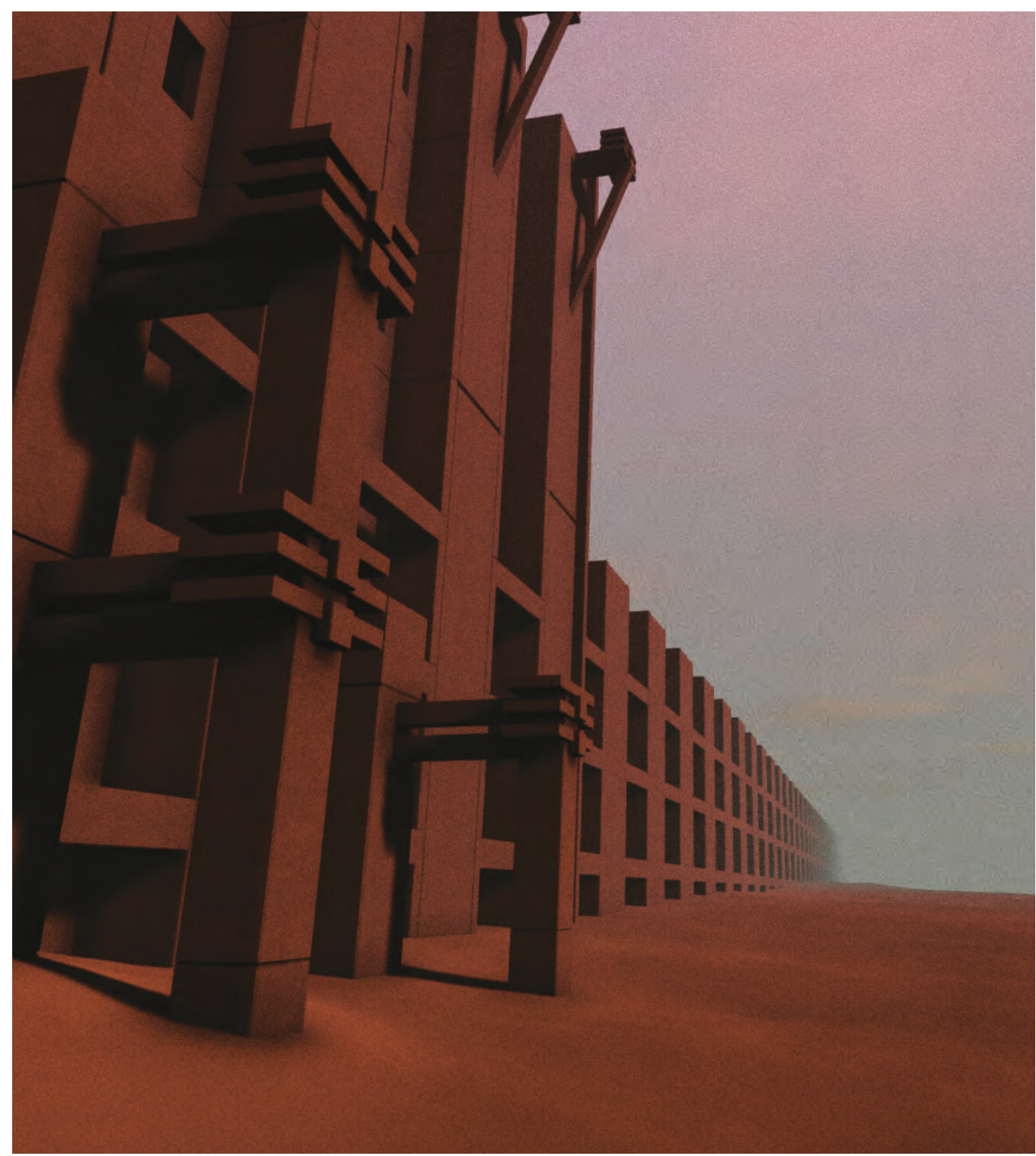


Reverberating footsteps immerse you in Naissancee's lonely structures. Hear bio-mechanical hoses and stone structures are replaced with monochromatic gray forms. The lack of colour allows the white lights to act as waypoints for the player. Dark shadows are cast across most surfaces of the gigantic rooms and buildings. The abstract forms the environment is composed of give away few hints as to what the purpose of these places were. An unsettling feeling creeps over the player when they find an empty dinning room in this place that was supposedly empty. After hours of listening to only the sound of the players footsteps the idea of hearing anything else is terrifying. The player reaches a point where they hope they are alone in this place. A close control of audio will affect how the space is interpreted by the player. As in architecture light will define volumes of space and shadows give the forms emotion.

EIG.7 NAISSANCEE IN GAME SCREENSHOT

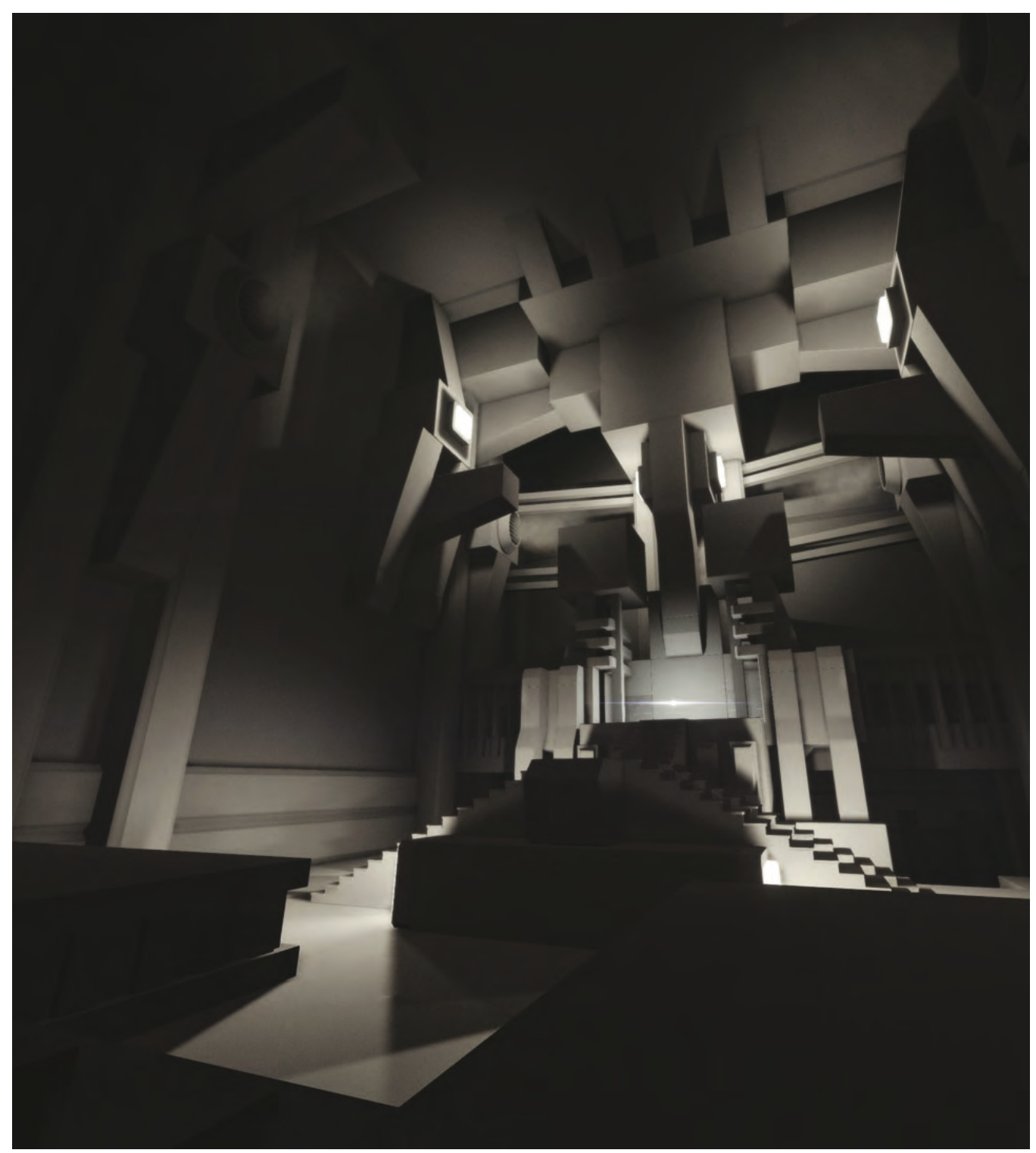


The endless fractal environments add to Manifold Garden's playful quality. The first time the player leaps from the edge only to fall back onto the same ledge or shift gravity to begin walking on the walls opens the player's mind to enchanting possibilities of space. Colour is used cleverly to differentiate the levels that the player is currently completing. The gravity shifting and looping space game mechanics while simple are enough to allow for many creative puzzles. Abstracted forms of nature are used to tell the player a story of removing an infection from the architecture. Elements that are dangerous are indicated by a black void. Manifold Garden is the first example in these precedents that allows for player choice of which order they will complete the games objectives. FIG.8 MANIFOLd GARDEN IN GAME SCREENSHOT

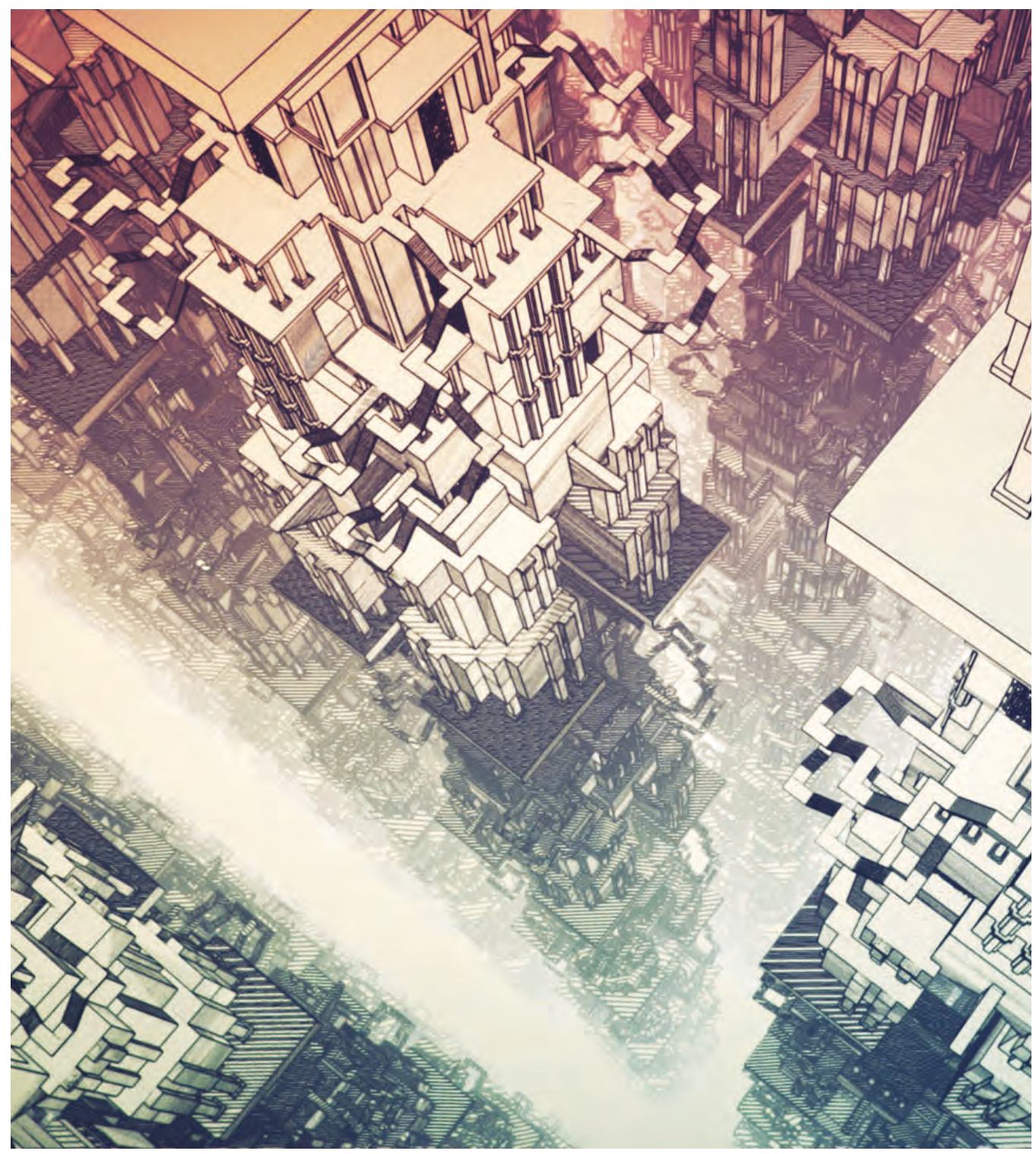


Narrative is at the forefront of Beyond Crimson Stars where you dive into a loved one's mind in order to experience their life. Here the architecture and visual presentation become analogous to disease and fading memories. Audio narration adds to this games story in combination with abundant symbolism. Bowling pins replace adults and thoughts are represented by floating text above buildings. The game combines abstract representation with the experience of entering a compromised mind. A pixelated filter is used for almost the entire game to obscure every element the player sees. This filter works as a metaphor for the fragility of memory, but it also forces the player to move around the space to make out forms. Visual obfuscation leads to the player spending more time moving about and observing the environment. If the player wants to attempt to decipher the intense narrative and symbolism, then equally intense observation is necessary. FIG.9 BEYOND CRIMSON StARS IN GAME SCREENSHOT

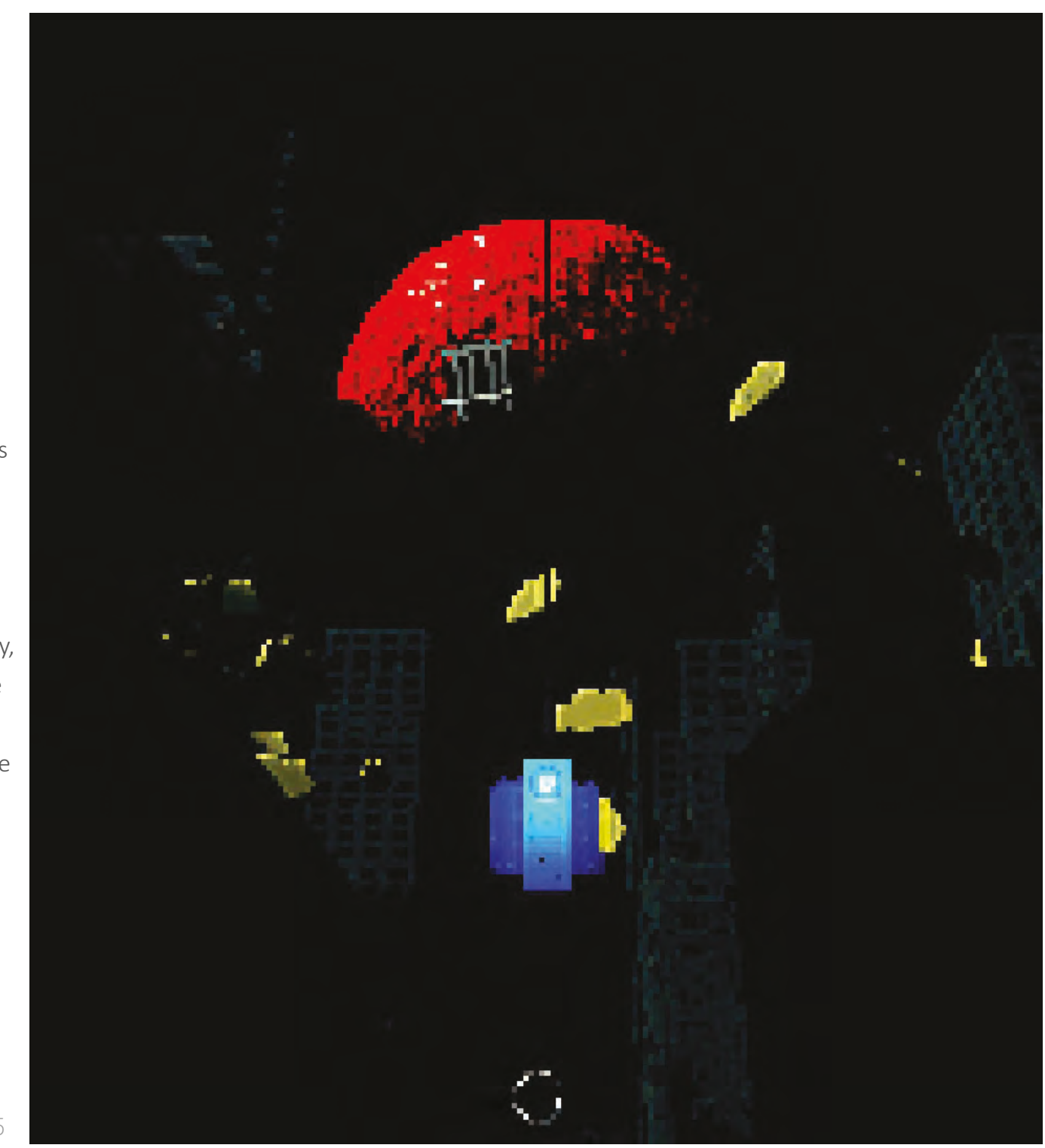


Inside is a polished game from an experienced video game design studio. Inside has advanced character animation combined with the tight controls. The player can see the character struggle to climb over a fence or panic as they gasp for air below water. The game is presented in 2.5 dimensions meaning the players character is locked to left and right movement, but the environment extends into both the foreground and the background. Inside serves as an example of highly refined simple game mechanics (walk, jump, pull, push) but they are independent from telling the story. The most interesting moments in the game are when the players character can control other characters in the game, a mechanic involved in the games twist ending. Control is exemplified here as one of the most important components of game feel. The game also serves as an interesting illustration of space taking place along what is essentially an impossibly deep architectural section.

FIG.10 INSIDE IN GAME SCREENSHOT

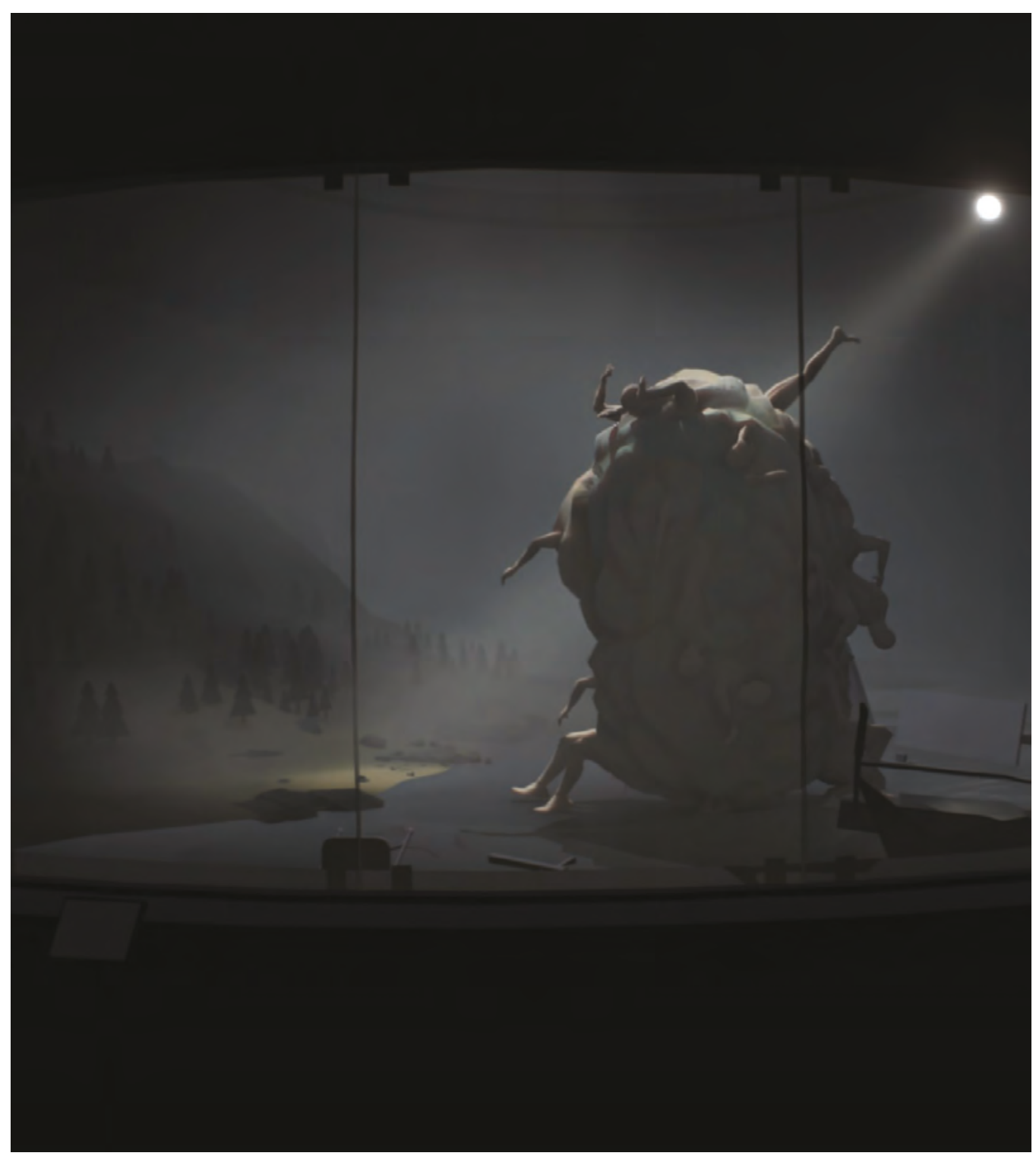




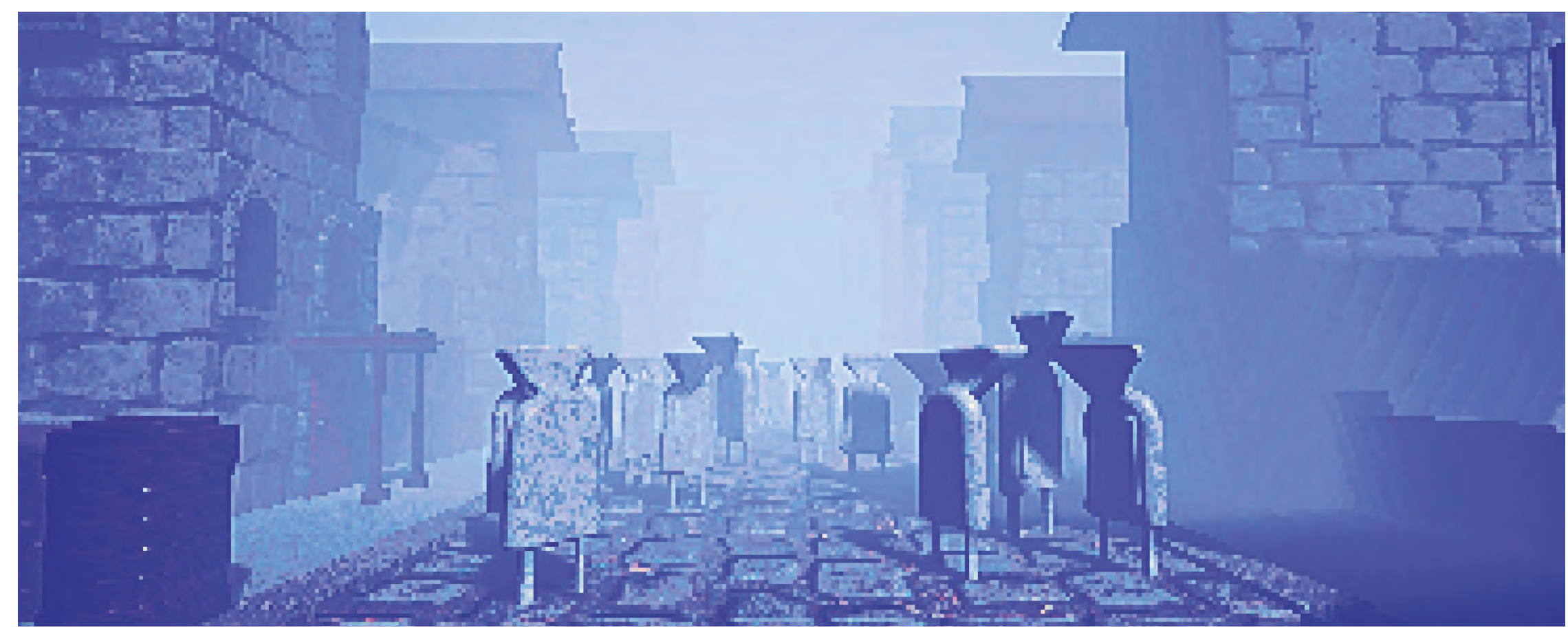

Each game employs its own strategies in terms of representing their themes. Common among the five games is a level of abstraction from the actual world. They employ this level of abstraction to varying degrees from approaching realistic to densely pixelated. The most affective use, to me, of representation was Beyond Crimson Stars due to the connection between its representation and its narrative. While things can be easily missed, and it is hard to make almost everything in the game it feels appropriate for the world. While I believe all five games to be outstanding examples of both representation and experience in video games the experience of playing Beyond Crimson Stars stuck with me for days afterwards. This made it the ideal candidate to describe for the reader the experience of playing through a games space. This style of description will be necessary to describe my own game.

Fig.11 Beyond Crimson Stars in Game screenshot 


\section{Beyond Crimson Stars: a walkthrough}

Video game designer Ben Lunato self-published Beyond Crimson Stars on January 19th 2020. Known for his distinct abstract style, he is a prolific game creator with 31 titles currently available. Beyond Crimson Stars is for sale on the online marketplace Steam. When asked to describe the games narrative to a customer, Ben responds by saying "One of the themes of the game is blending reality with fiction with dreams ideas feelings and TV so what's real and what's not real is not a hard truth. Something might be truthful without being true. I'm not too sure that my intentions matter that much now" (Lunato 2020). His answer is as unclear as the game's visuals. The experience of what it is like to play through these layered and symbolically dense spaces is what makes the game so memorable. The following log of my playthrough is an attempt to describe what it is like to be immersed into this abstract representational experience.

The first thing that I notice when I awake in the dimly lit room is that my vision has become completed pixelated. I can barely make out the stain glass window which seems to depict a rose ten feet above the floor. Ultra-bright lights and blurry symbols shift resolution as I look around trying to find my bearings. These are the opening seconds of Beyond Crimson Stars. This feeling only lasts until the first bit of narration begins.

Fig.12 Beyond CRimson Stars entrance to the mall screenshot

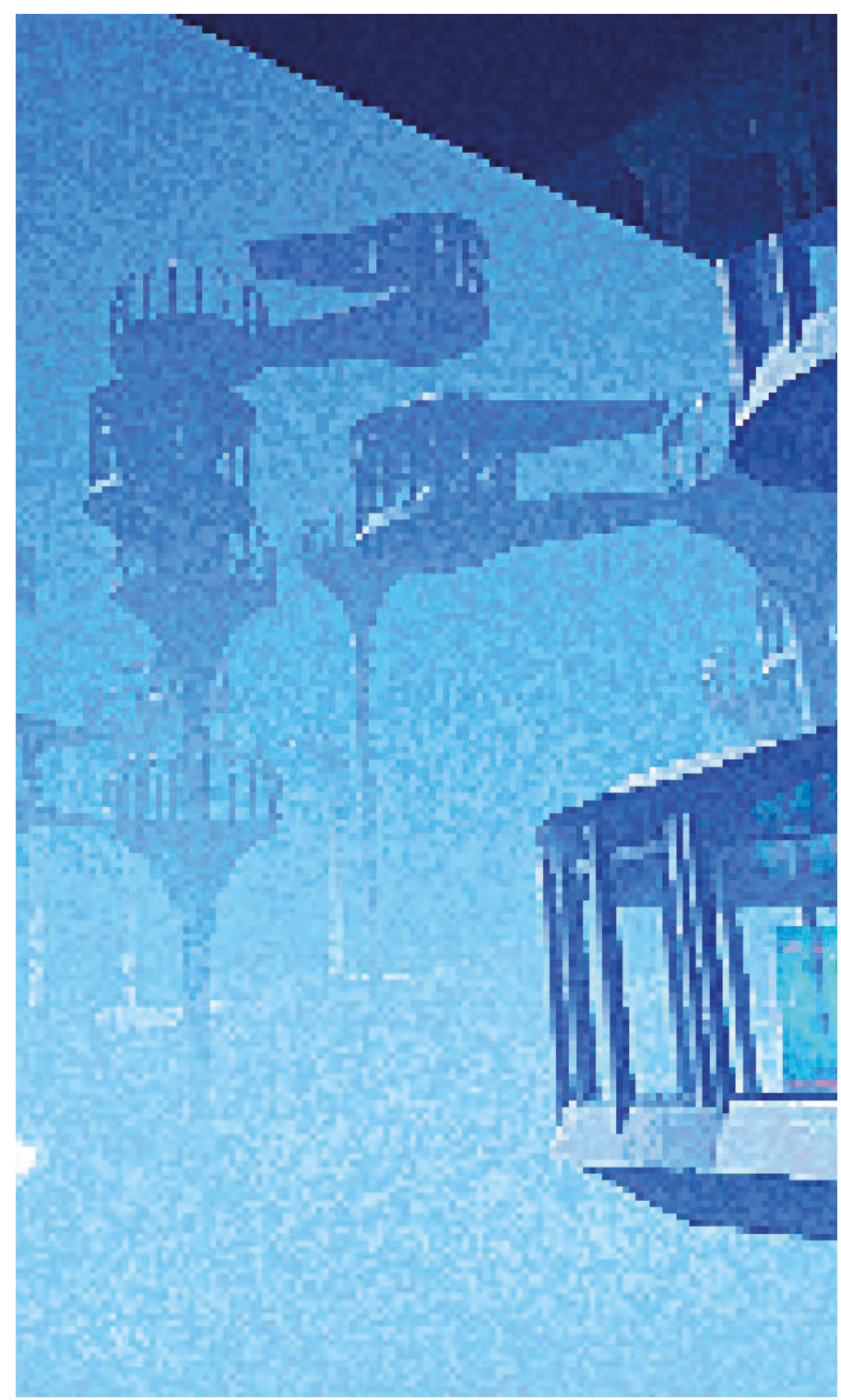


As I begin my first playthrough I am introduced to the games guiding narrative. I am taking my father's recently deceased and damaged brain to the local mall. There I will be able to experience the memories that were once his. The games highly pixelated visual noise begins to resonate as I start exploring my father's faded memories.

Within the mall I see four thresholds, each providing entry to a damaged memory. The deafening sound of waves erupts from the red lit threshold. Within I am greeted by rows of chrome tombstones resting within the blood red rock. They reflect a light into my eyes from a sun that does not appear to hang in the crimson sky. Metallic vultures shriek insults and erupt into white light from atop the largest of the chrome structures. The sounds of crashing waves continue to drone through the entire journey until a narration along a misty bridge explains the noise as a distant shore.

The second memory has me awakening again, this time from a stasis pod aboard a starship. A cheerful Al is glad to see I am awake and immediately uses this opportunity to show off some of the poetry it has been working on. As I follow the Al around the ship the poems spring into existence. Shapes and colours surround me a on all sides illustrating the metaphors

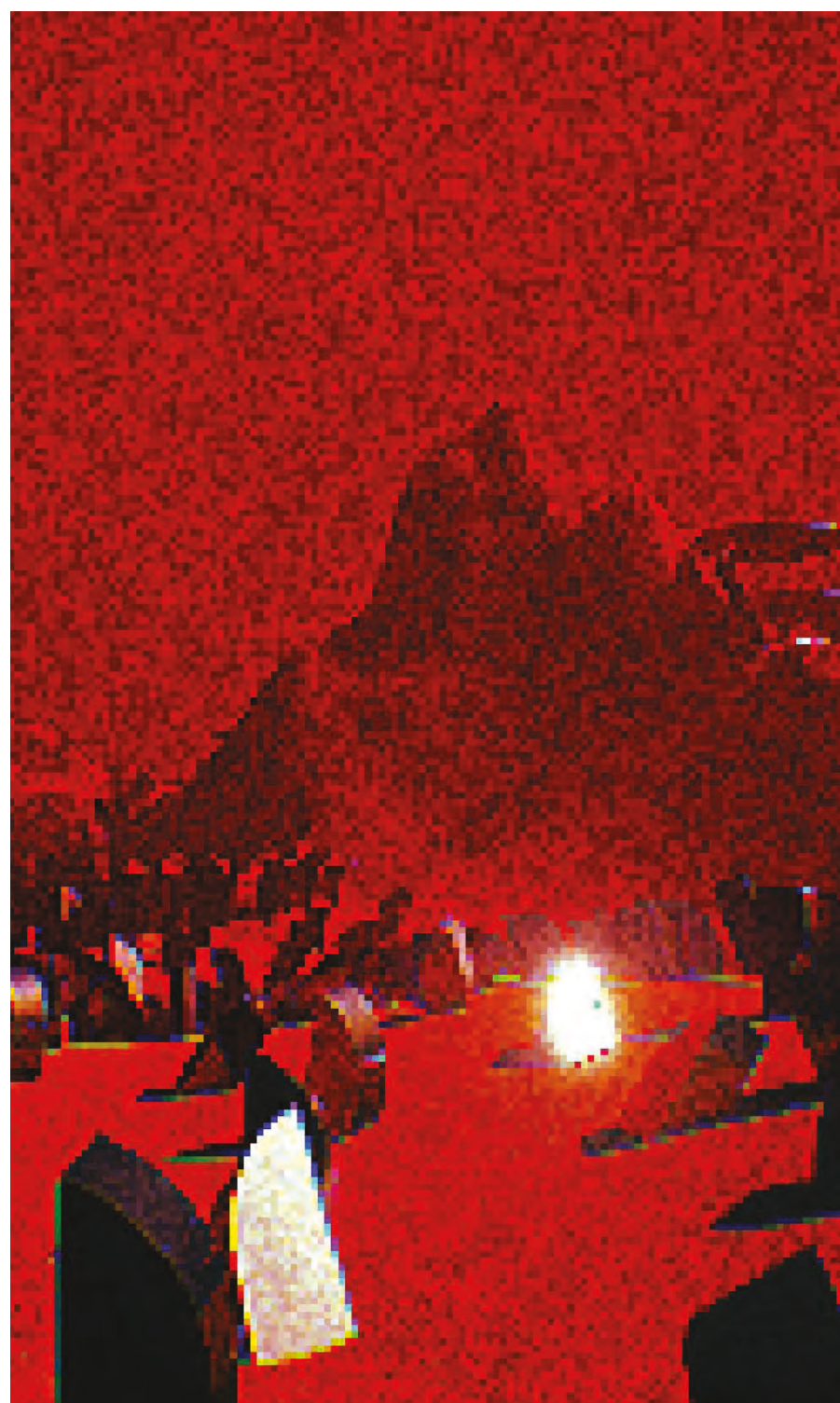


of snakes and seeds that are being rambled by the robot. As quickly as the poem started it ends from embarrassment. The Al switches to an abstract tale about some village folk that finishes with a clear reference to real world racism. Hooded figures and burning crosses end my walk through this town. Back aboard the ship the Al encourages me to end my own life. The gun proves to complicated to use for my character whose only ability is to walk and observe. I drink some bleach and walk into an oven but none of my suicide attempts have any real affect on the character I am controlling. The Al becoming irate with me as each attempt fails. The robot yells at me for attempting these actions of self harm. It is becoming clear to me as I leave this memory through a set of building facades along a streetscape that I am inside a guilty conscious. All around me are attempts at creative expressions that seem to have never manifested into anything I found substantial. I jump from the plank of a pirate ship and wake back up in the mall.

It is nighttime on the streets of the third memory. A black sun casts a dark blue green glow on metal wreckage and decayed buildings. The city gives way to a misty swamp. The only way I can make it through to the other side is by following the red lights. Scaffolded walkways lead me out of the swamp and as I walk towards what appears to be an audience. The journey is narrated in time ending as I arrive. The narrator ponders how a person's worth can be measured FIG.14 BEYOND CRIMSON StARS BOtTLE OF BLEACH SCREENSHOT

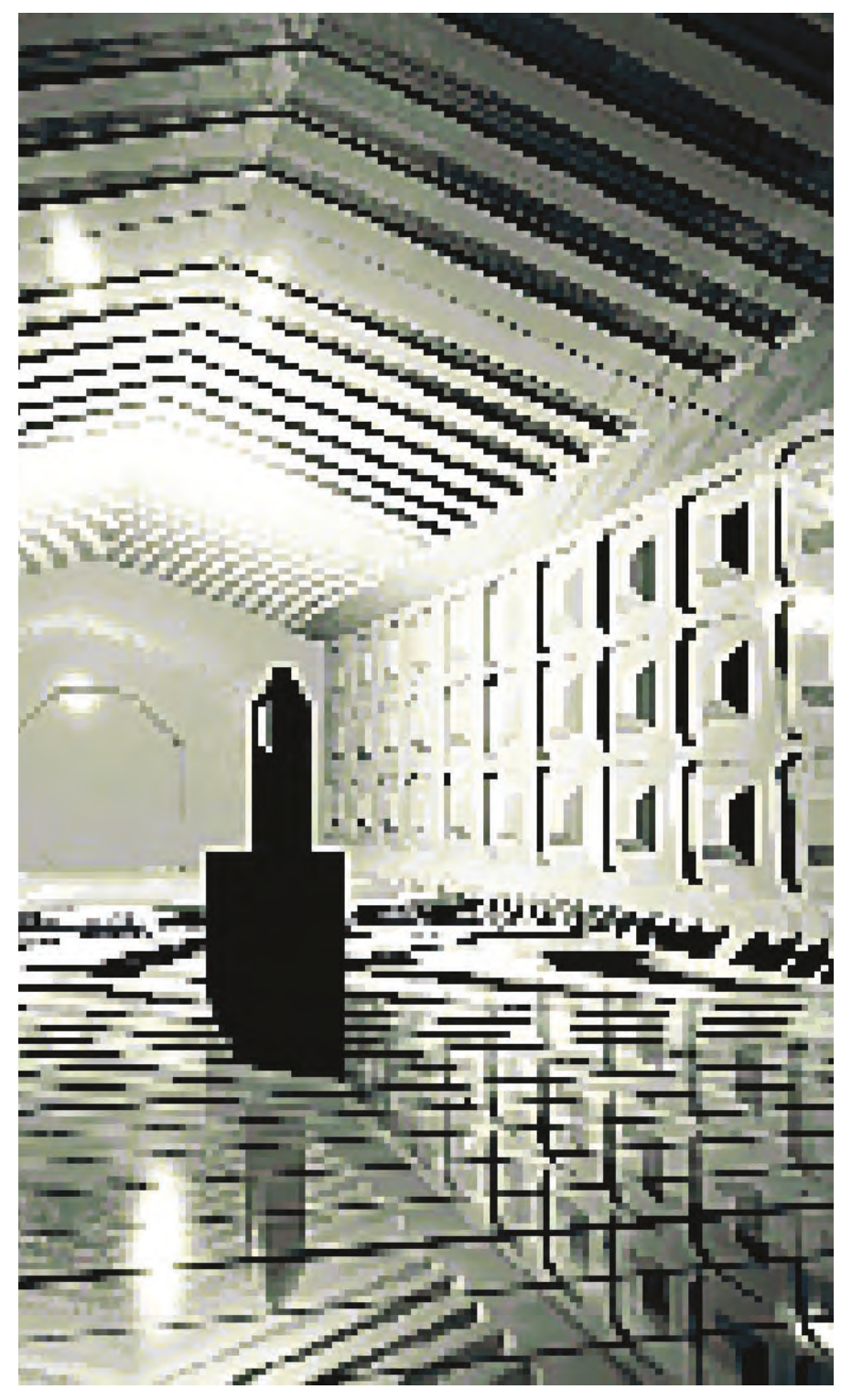


by what they can do when a tools worth is measured the same way. As I join the audience the scene switches to an interview talk show. I can no longer move and forced to listen receive news of their mother's terminal illness. It is a deeply unsettling moment of voyeurism as I watch the character models begin to glitch. An intentional choice by the programmer echoing the discomfort I am feeling.

The final threshold of the mall takes to me prison visiting room. I confront the figure on the other side of the glass speaking to them. Before they respond my perspective is shifted to theirs. I am now in control of another person, what seems to be my son. I turn away from my father and proceed back into my prison. Here I am taken increasingly abstract memories talking with a friend who points out the futility of meaning in the world. A car crash brings me into my parents living room, each a bowling pin sitting on the couch. They criticize and yell at me the longer I am in their presence, so I retreat to my room in order to stop the barrage. Leaving the house slurs are floating in the sky of my neighborhood. I am walking to school and the slurs and voices reinforcing them grow louder. At my desk I see the walls replaced with targets. I am confronted with the disturbing realization of what my character is about to do or had once thought of doing. My already obfuscated vision becomes worse as the filter switches to black

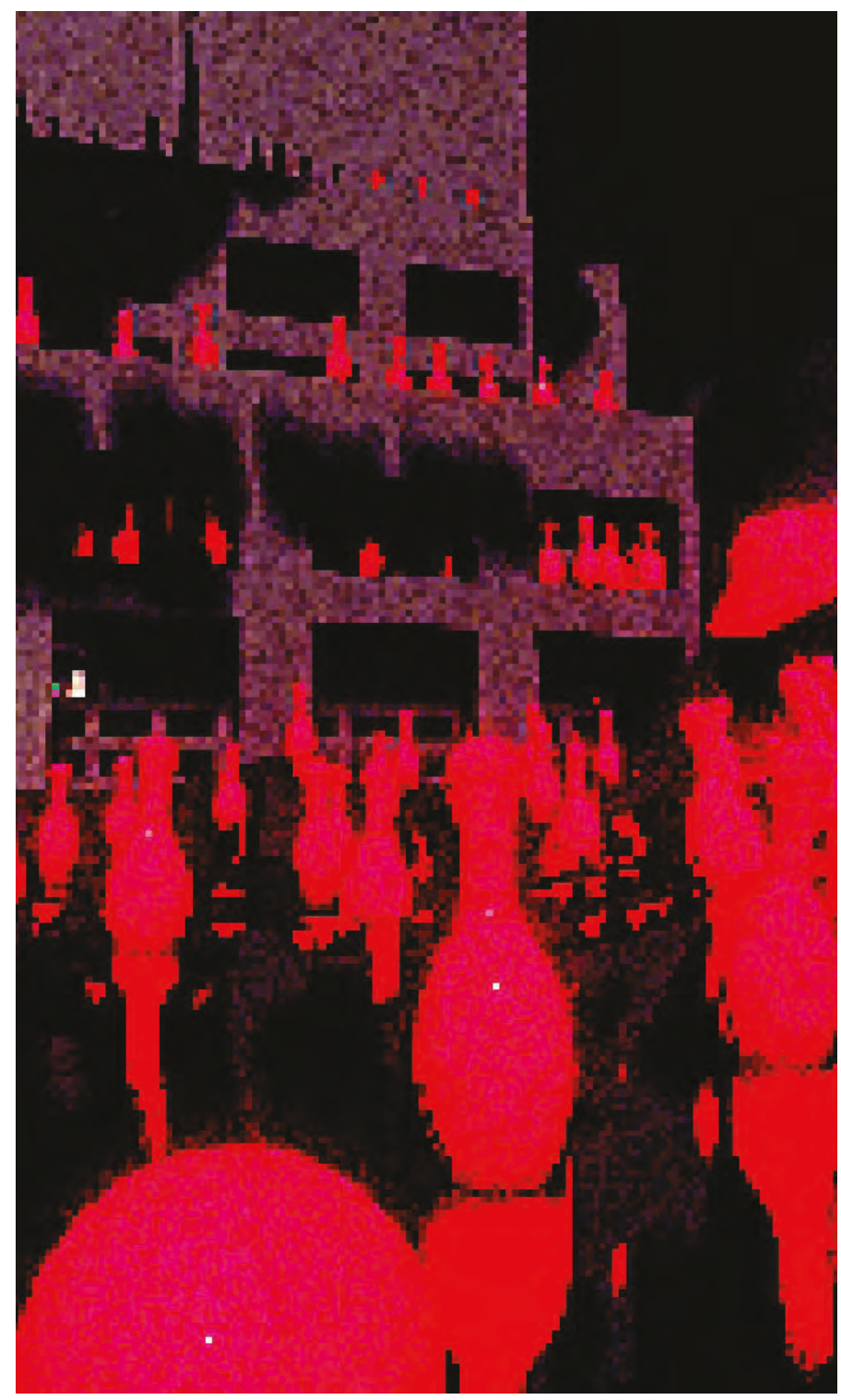


and white. I am now barely able to maneuver over the scattered bowling pins littering the hallway. I escape the horrible scene into the woods where the memory ends as I confront my father and fade to black listening to the radio.

Back in the mall a final door has opened. It is a steep climb to get to the source of the golden glow above the tables and chairs. I am treated to a warm narration by my father. One about memory and moving on after death. As I approach my childhood house, I get my first glimpse of a clear image. A wall not made of plaster or brick but made composed of old home videos. The fireplace is made from a smiling child playing in the pool. The living room wall is an old clip from a baseball game. As I proceed to the back of the house I am filled with warmth and happiness. The components of the house float in a state of deconstruction each element hosting memories of a child with his mother and father. I look around savouring this architectural metaphor. After what seems like no where near long enough my father's voice reminds me that I can stay here forever, or I can choose to move on. The choice to move on is not an easy one, after a journey filled with horrible imagery it is tempting to remain in this house forever. I back into the final doorway keeping an eye on the memories as long as possible.

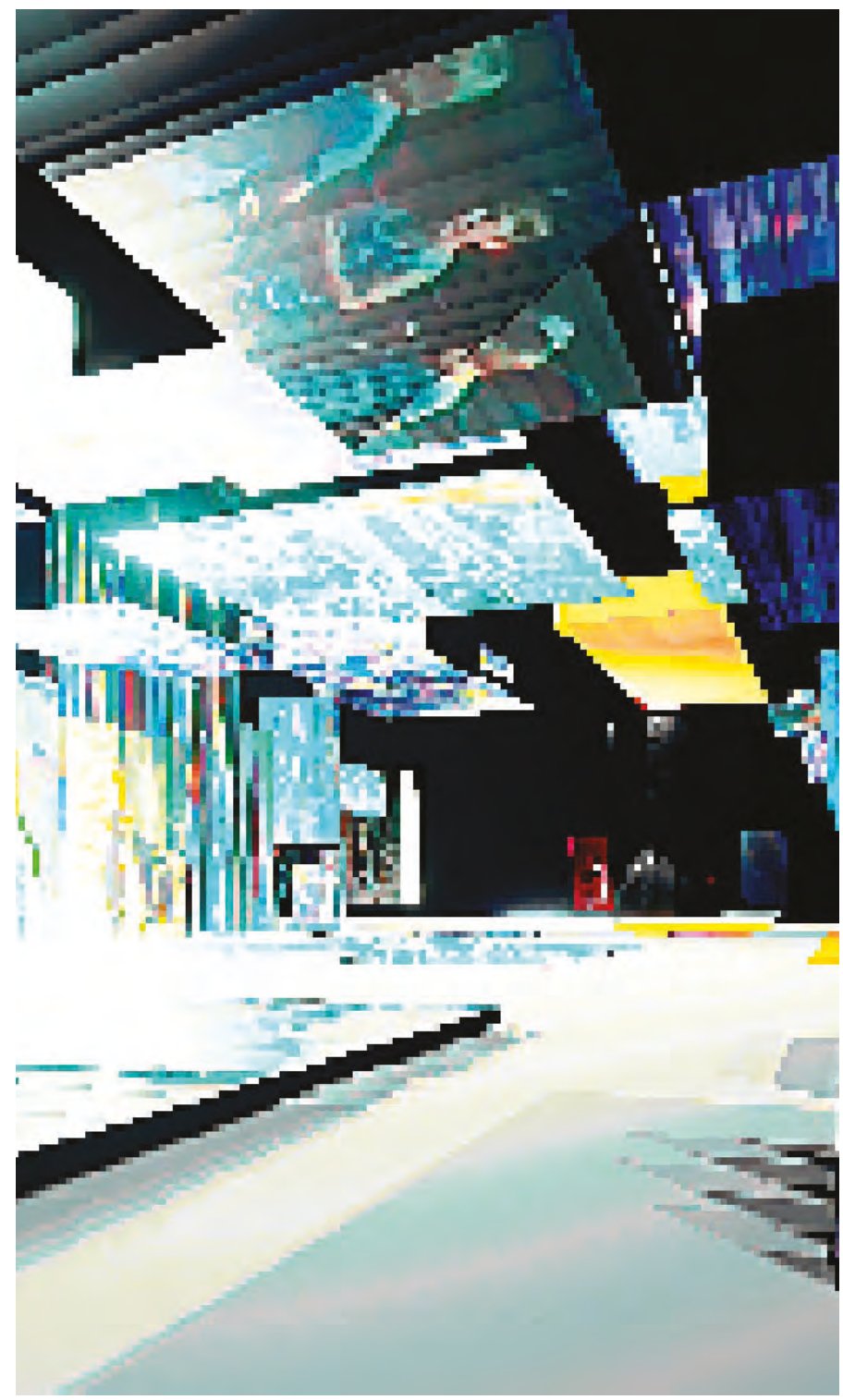




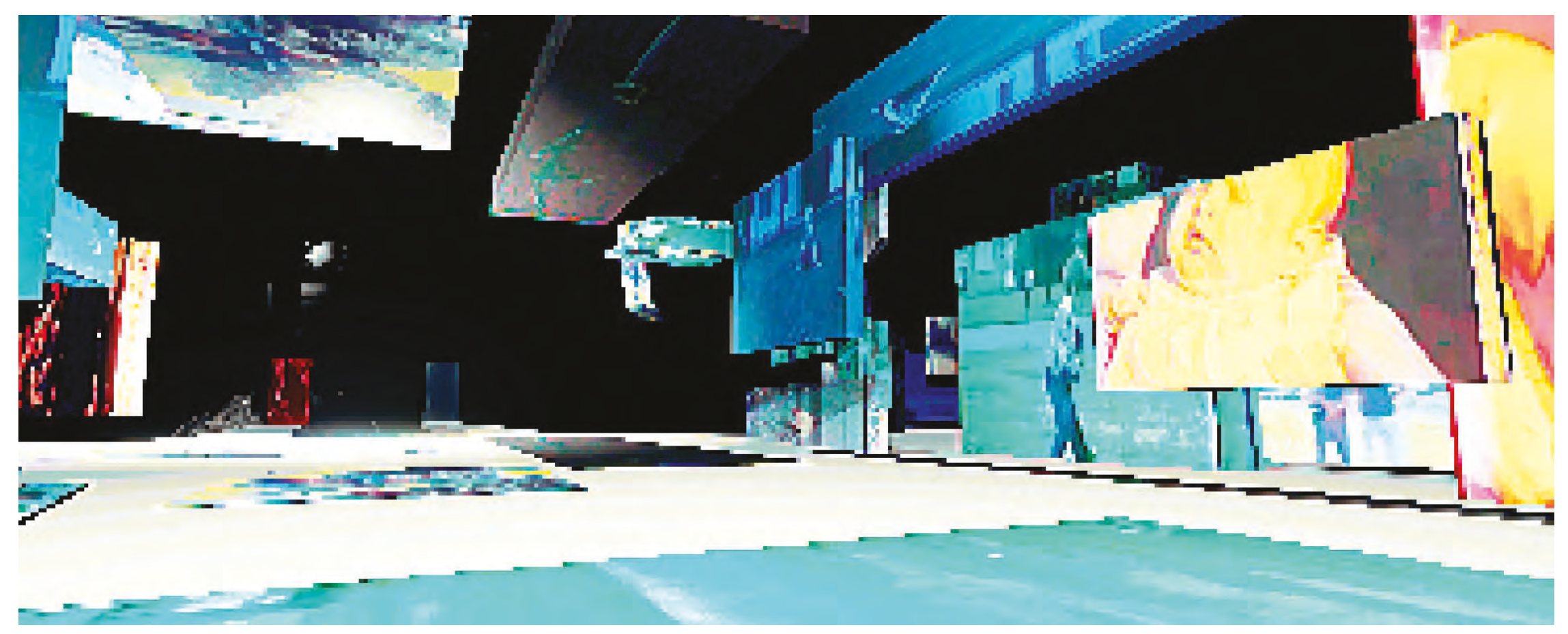

The final moments of Beyond Crimson Stars remained with me for days afterwards. It is rare to experience such an intimate moment in any form of art. Ben Lunato allowed me to walk through feelings, memories, dark moments, and possibly some of the happiest. He achieved this through the medium of video games. A medium where he could combine poetry and narration with virtual space and experience. The power of the final moments comes from the faded and unclear filter on the memories up until that point. The darkest of thoughts can barely be recalled while my happiest persist. This is memory is conveyed as spatial metaphor and the characters perception (my perception) of this space. It ends with a choice poised by the characters father. You can remember for as long as you need but in order to move forward, you must move on. I have no desire to replay the game and see what happens if I choose to stay. I moved on.

fig.17 Beyond CRIMson StaRs house of MEMories screenshot 


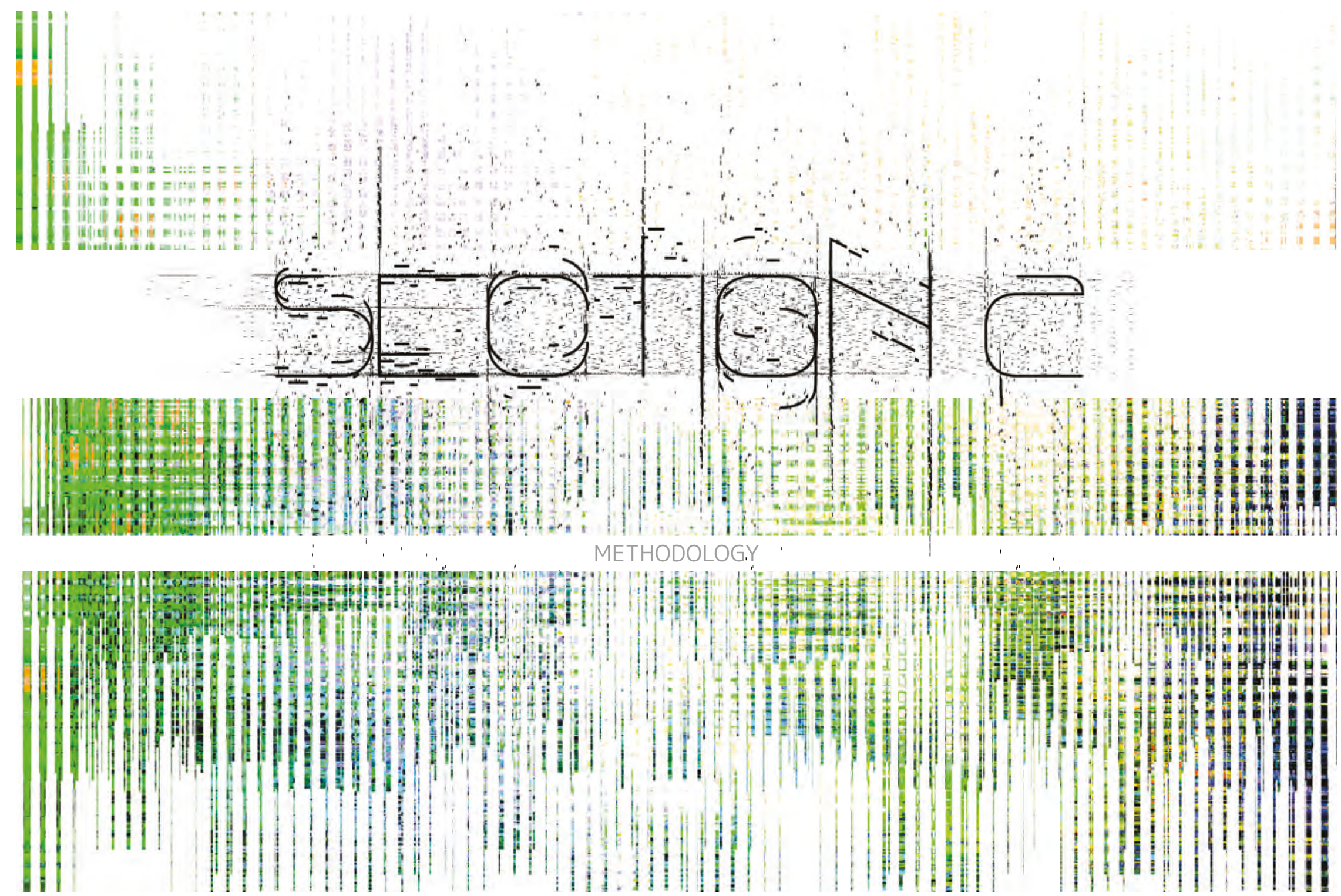




\section{The Game Body Diagram}

The player and the player character are in constant sensorial communication while playing a game. A player is inhabiting the body of the player character. The relationship can be this simple or it can go much deeper. The game Inside, for example, has you playing as a young boy who on occasion inhabits the mind and body of another person, who on occasion can inhabit yet another person. The end of the game reveals the character was playing as a mass of congealed bodies inhabiting the young boy's mind in order to free itself. No matter the narrative relationship between the player and (player) character some senses are shared across the screen, and some are not. When playing a game from the First-Person perspective, the player and the character share the same vision. What the player sees is often what the character sees. On a computer the player normally moves the character with keyboard inputs and looks around with the mouse. Hearing is another sense that is often shared between player and character. Sight, movement, and audio are the factors that contribute to the immersion a player has with the character and by extension the games space. Haptic feedback is beginning to be implemented in technologies like Valve's Index where each finger is recreated in the game allowing you to pick up and throw objects. Pain is a major element in many games but for now that is only experienced by the character. A player while controlling a character is in constant dialogue between two bodies mediated by the screen and inputs used to control the virtual world.

Two torsos are faded into each other at the top of the diagram. They pixelate into each other, blurring where they might connect and where they might be separate. W, A, S, D, and Space Bar are the standard inputs for FPS (First-person shooter) movement. Below are the minimal representations of the keyboard. Gestural line's mapping mouse movement are scattered around the diagram, the standard way of seeing in an FPS game. The code that converts the inputs from the player's body, through the screen, into the character's body appear as the structure the image is built on. Much of the code is pixelated and distorted. This code is only apparent to the player when it malfunctions. It is at this moment when what is known is a glitch disconnects the player from the character, a glimpse of the infrastructure the virtual world is built on. The glitch has the potential to be a didactic experience in a game. Most games intend on hiding any element that would reveal that it is a game constructed within a virtual reality. The use of glitches in the thesis game will allow for the coding infrastructure to be revealed and experienced. 


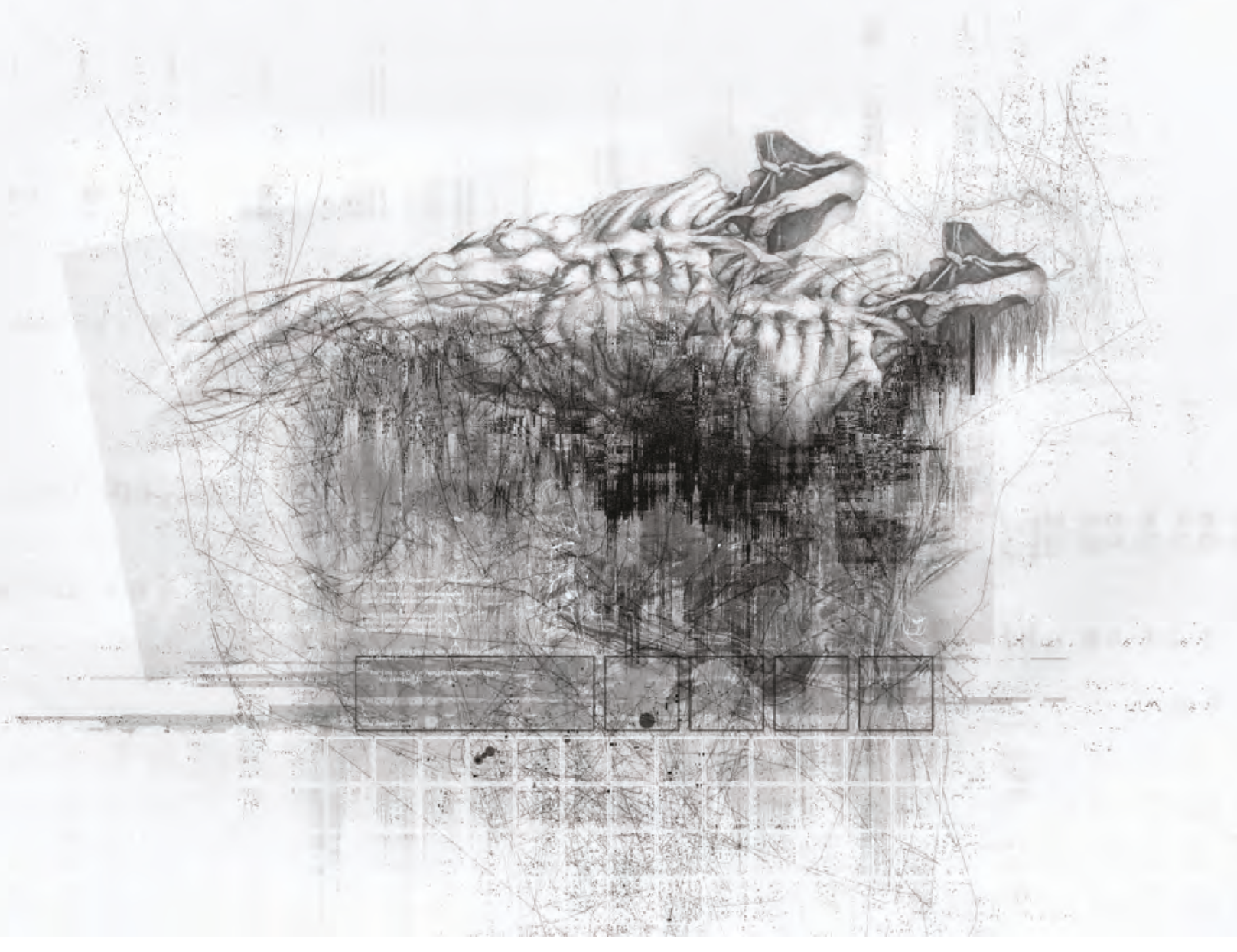

FIG.18 GAME BODY DIAGRAM 

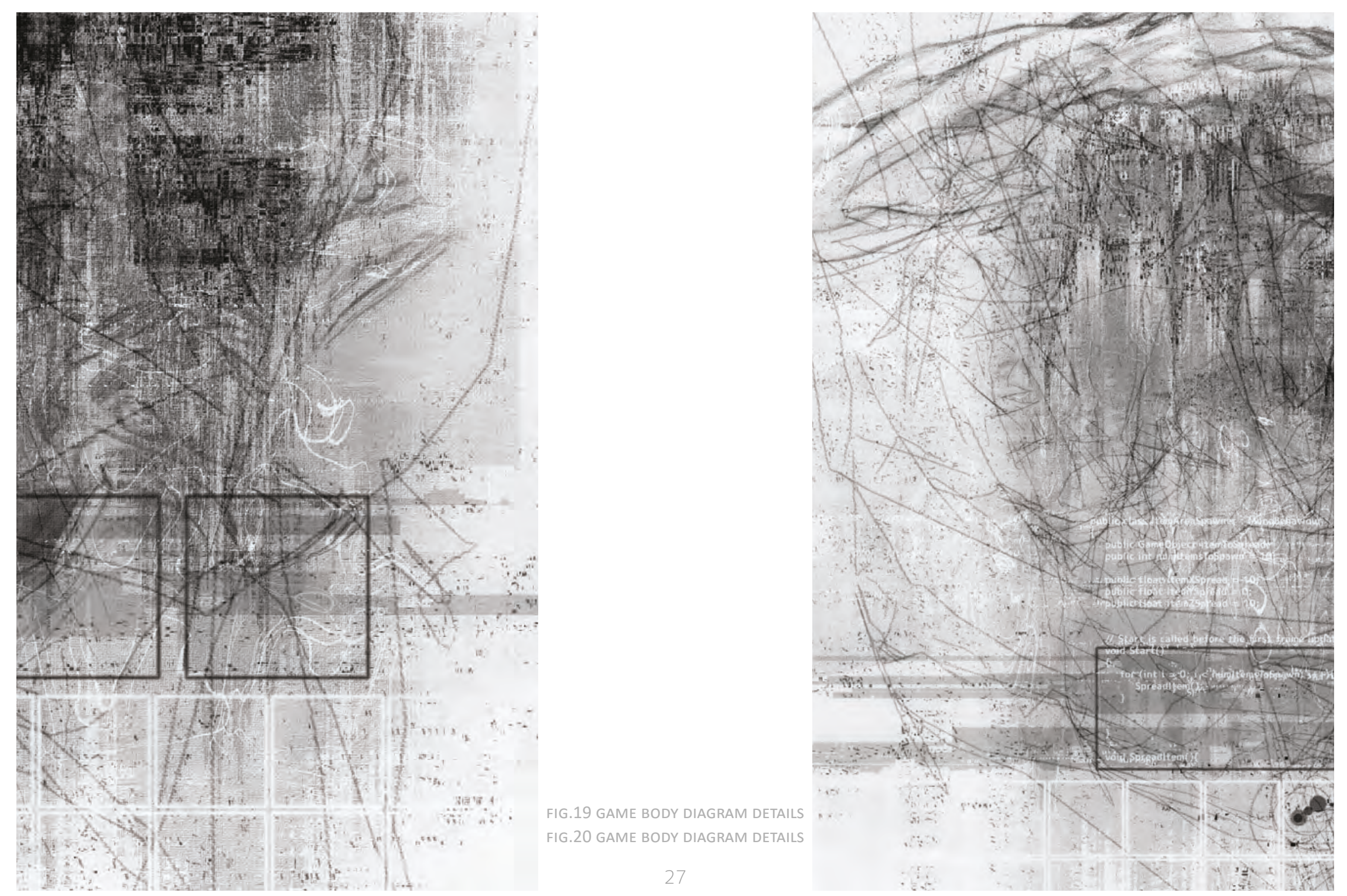


\section{The Game Space Diagram}

PRIMA Official Game Guide on Tom Clancy's Splinter Cell Chaos Theory was used to inspire and create the game space diagram (Loe 2005). Official Game Guidebooks used to be the source for all detailed information related to a videogame. They were filled with level layouts, plans annotated with the location of important game items or enemies to be avoided. Axonometric diagrams were accompanied with paragraphs detailing strategies to get the upper hand on multiplayer opponents. Architectural notations were used in conjunction with game graphics in order to educate those interested in the best way to approach a difficult section of the levels. Splinter Cell Chaos Theory, a stealth action classic, was known for intricate level design. The plans and axonometric diagrams in this book were used as the starting point for creating the drawing that developed the game space of this thesis.

A key notation that stands out in the level guides is the line connecting a 'door A' from one plan drawing to 'door A' in another plan drawing. This line indicates that if the player character enters door A, they will exit at door A in the adjacent plan. In game space this distance does not need to be the width of a door frame but could be any distance. Games often use these thresholds to transport the player to an entire new game space. Chaos Theory has the character go below deck in level 2's cargo ship. The space above deck in relation to the space below deck is inconsequential and can be completely moved to any part of the virtual world, the door between the upper and lower areas serves as a 'portal' to an all-new location. The architecture of game space does not need to follow any of the limitations of actual space. The transitions between game space areas can come with changes of scale indiscernible to the player. The distance between the entrance and exit of a doorway can be a million miles or it could exit in the same room you left from a doorway that is half the size. The relation of the scale of the characters body to the scale of the space remains important as in any actual space. These spaces however do not need to adhere to any actual world physical laws or limitations. 


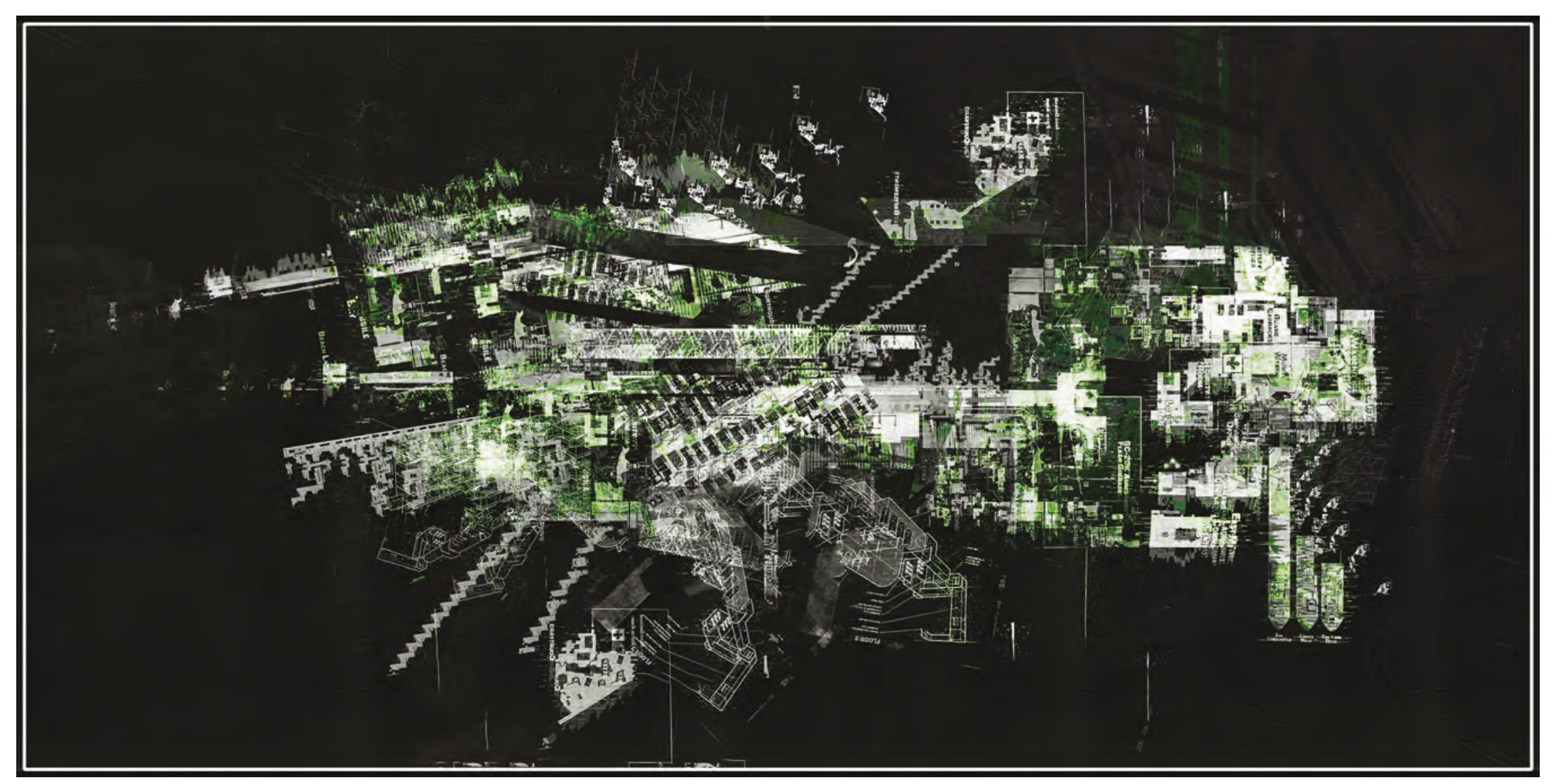

FIG.21 GAME SPACE DIAGRAM 


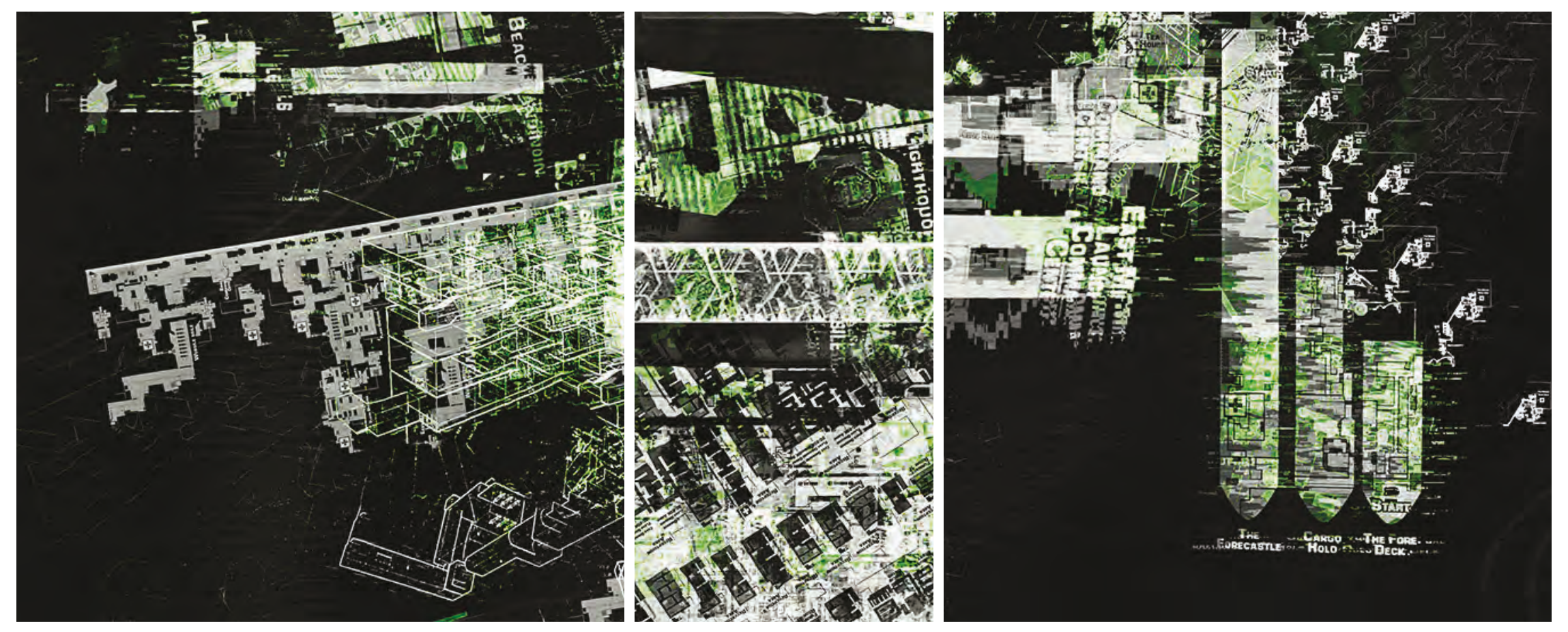

FIG.22 GAME SPACE DIAGRAM DETAILS

FIG.23 GAME SPACE DIAGRAM DETAILS

FIG.24 GAME SPACE DIAGRAM DETAILS

With these factors in mind the drawing began to take shape on a black background. Incorporating the plans from the levels of Splinter Cell but rearranging the doors and stairs changing where they led. The third level's US Bank vault heist rooftop entrance now leads to the eighth level's Seoul Apartments. Doorways cross continents and scales rapidly change on the page. The coast of the tutorial level's lighthouse is multiplied and stretched, repeating towards infinity. Axonometric diagrams of the multiplayer levels are stretched across the plan, their notations now crossing boundaries between a games solo experience and its cooperative one. Edges of the plans begin to pixelate into one another and fade out into the black. The background becomes a warped and pixelated distortion of what the plans began as. The game's plans cross the boundary into a completely different game as a hallway from Beyond Crimson Stars stretches away into its own world 
Mechanics of Drawing

Drawing is linked to the process of design work. In the fine art world, until recently, drawing was used in service of painting. Forms and composition were developed and placed on the canvas through drawing and scenes from nature were recorded to bring back to the studio. The definition of drawing is ever expanding in both the field of architecture and art. Architecture employs drawing as both a process tool and a final product for construction, as does this thesis.

The body and space diagrams are considered hybrid drawings, a mixture of both physical and digital techniques. Importantly the drawings were not created with an end goal in mind. The process of drawing the diagrams allows the author time to consider what the implications of the marks, symbols, lines, and colours mean to them and convey to others. Transitioning from sheet of paper to the computer is not as simple as scanning a drawing and dropping it in photoshop. There is a complete transformation of how that drawing is now perceived and what it is made up of. In this example the sketch has moved from a physical space, graphite on paper, to a digital space, an algorithm interpreted by a program. The image is now represented by pixel approximations of the lines on the paper. Here a loss of information occurs due to the computers use of a screen and the camera's ability to record an image. The thing that stands out most in this process is that the image is no longer made of marks, symbols, lines, and colours but strings of code. A language that machines speak so that we may see. The code is the language of the computer and to consider the implications of representation and its experience trying to understand coding was necessary

FIG.25 HYBRID DRAWING OF LEVEL 2 CONSISTING OF HAND DRAWING,

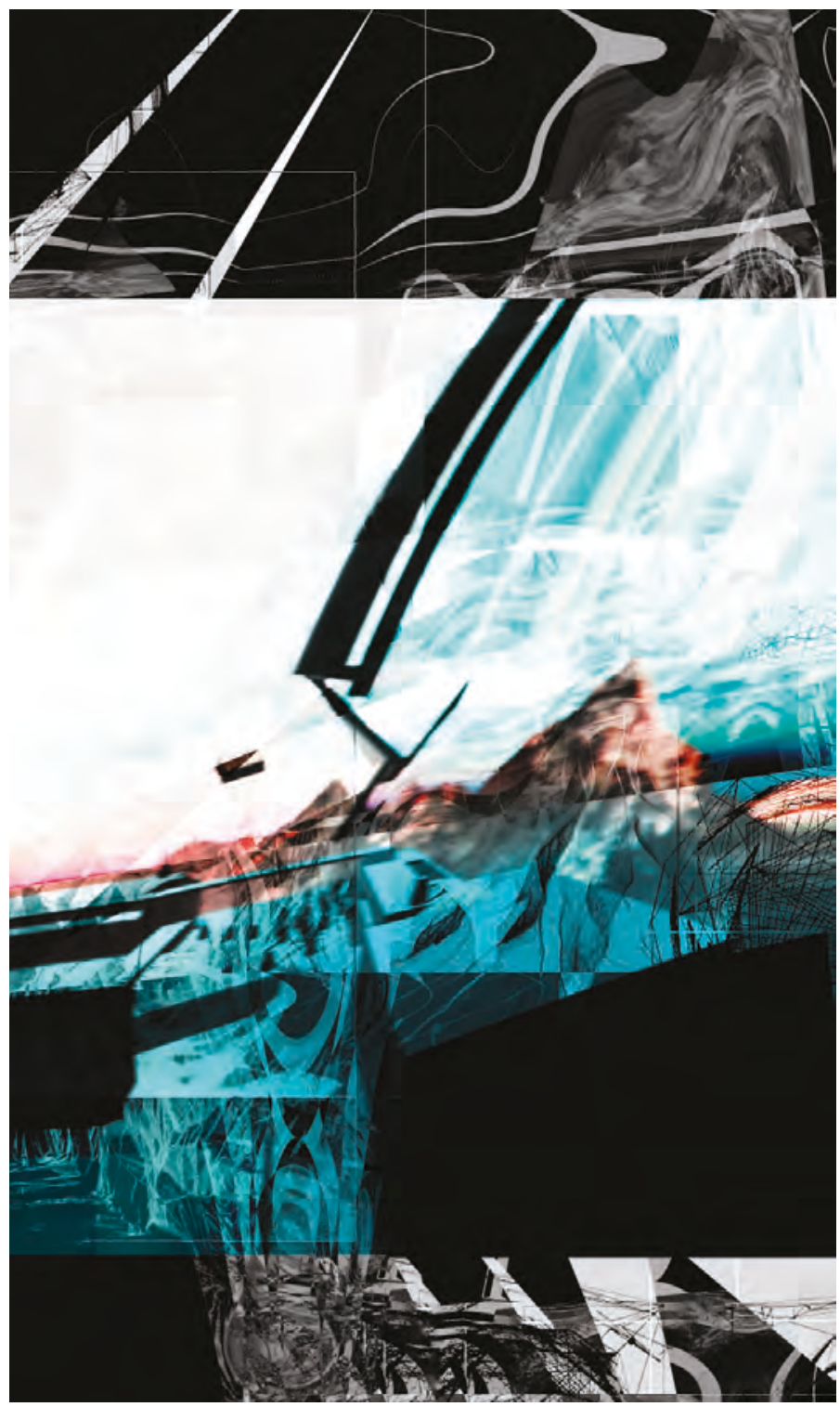




\section{CODING}

Learning to write basic C\# coding language would not have been possible without the help from online communities and game developers. Resources for learning are abundant on online video platforms and libraries. The speed at which the Unity Game Engine is updated means that within a year some tutorials can be out of date. I made the decision to use the 2019 version of the game engine due to the abundance of available tutorials. Many weeks were spent wrestling with the concepts of learning a programming language as someone with no experience. My main struggle was my lack of ability to experiment with writing code.

When drawing you can make a bad mark on the paper and observe what you have done wrong in comparison to the other marks. When learning to $3 \mathrm{~d}$ model you can play with the objects and parameters seeing what is possible and when the program is no longer able to handle what you ask of it. When learning to write visual code you write it, save it, open Unity, run it, and see an error that says the code does not work. Often the issue with written code is a syntax error, character out of place, or incorrect symbol. When something needs to be shown on screen or rendered in a certain way mathematics are needed. Coordinates and vectors are constantly called upon to define geometry in space. Here there is an opportunity to use drawings that have been rendered in Unity to illustrate what the code is doing. While initially getting a coded script to run can be a challenging, variables within that code can allow for experimentation outside of a visual scripting software. For example, a code that generates a random assortment of digitized sketches can be changed from 100 to 300 spaced out along an $\mathrm{x}$ coordinate 1 unit to 100 units long. These parameters can then be nested within further parameters to add complexity and intractability to the digital space. For example, once a player's movement can trigger an event, the speed of that movement, a variable, could be used to define a new space based on the number of geometries generated. As a designer I found programming easiest to understand as a series of events that could be enacted by the engine. The Unity Game Engine was chosen because of its reputation for being friendly to new programmers and game designers. 


\section{Unity Game Engine}

I began the design and development of the thesis game by deciding to build the project using the Unity Game Engine. Unity offers coding flexibility specific to game design that helps with developing a game that architecture design programs such as Revit or Rhinoceros 3D do not. The Unity game development platform is a tool for game developers to design and package games for multiple platforms. The Unity Engine is a series of engines based on algorithms that render virtual objects, perform audio, detect collision, calculate physics, and run artificial intelligence routines. The software is not meant to manipulate standard geometries, often these are done in other programs dedicated to the task and then imported into the Unity Scenes.

This workflow causes a separation between the aesthetic decisions and the game mechanics. Another way of looking at this separation would be that the Unity Engine brings the player experience that is being designed ahead of the aesthetic considerations. The program uses the C\# Language to write scripts which tell the Unity Engine what is going to happen in the game space. These scripts are the main way that the designer engages with the digital space that they are creating. They are dealing with digital events rather than with static geometries.

The Unity platform uses the C\# language instead of visual scripting like Grasshopper or Unreal Engine. The benefits of learning C\# go beyond game design creating a foundation applicable to other programming languages. The undertaking to learn coding having no prior experience was a difficult but rewarding one. In order to learn the coding concepts some representations of the code were developed alongside the script writing.

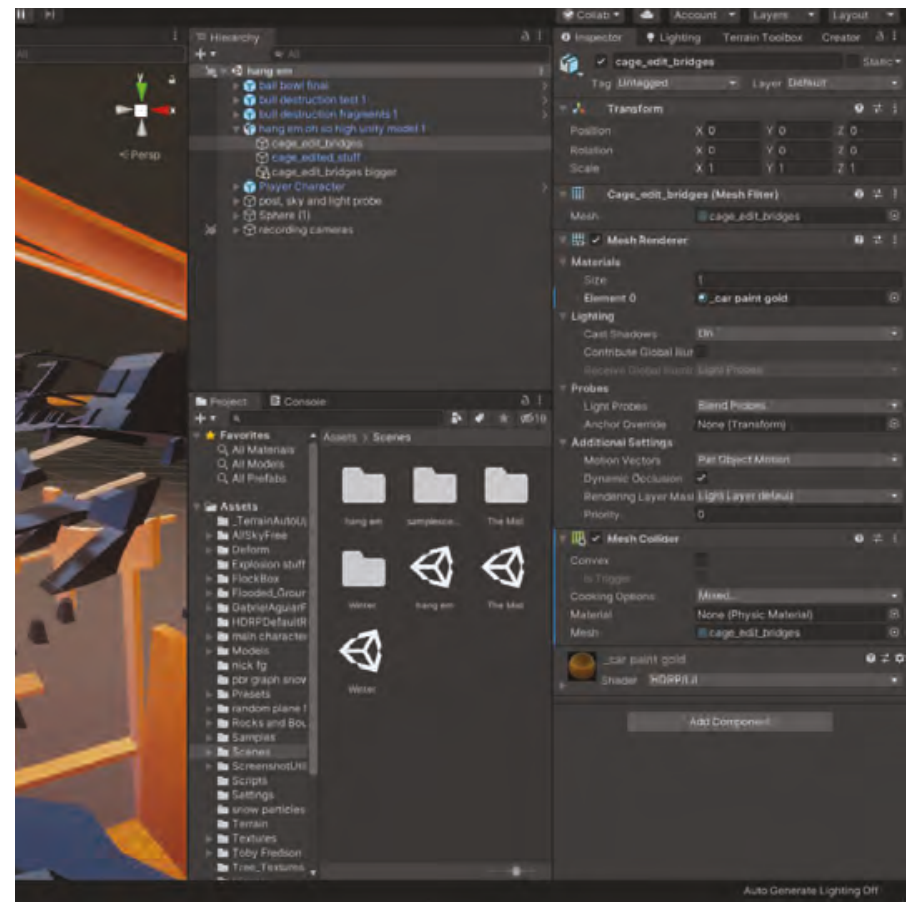




\section{Research Through Drawing}

To develop the project, it was necessary to come to terms with the constituent parts of designing a working video game. A critical part of a video game is the game mechanics. Game mechanics are the systems and rules that make up a video game's gameplay. A game mechanic defines how a game is played. Understanding game mechanics and how they could be implemented was the initial goal of my research. Game mechanics define the experience of playing a game meaning it is crucial I was able to understand and implement them. My case studies informed the mechanics that I began learning to code. Design in the digital realm can present infinite possibilities but there needed to be something tangible in order to represent and test what was being implemented. I would need a subject to test my game mechanics. Concurrent to my research and practice in game mechanics was my continued passion for hand drawing. An opportunity existed to connect my preferred form of representation with the new form being tested. Some drawings became test objects to run and understand my mechanics. Each drawing that was composed was a visual representation of a new game mechanic that was implemented. 


\section{Research Through Drawing: Game Mechanics}

1. Movement in Gamespace

Mouse-look

Jumping

Directional Movement

2. Interacting with Game Objects and Space

Picking up objects

Moving Objects

Throwing Objects

Dropping Objects

Physics and Force Based interaction with objects

Raycasting and Colliding to and with objects

3. Materials

- $\quad$ Shader Graph for morphing textures

- $\quad$ Random Selection of materials for objects

- $\quad$ Base maps

- Normal maps

- Height maps

- Pixel displacement

- Vertex displacement

- $\quad$ Post processing effects

4. Particle Systems

- Random emissions of mesh objects

- $\quad$ Predetermined lifespans of the particles

- $\quad$ Predetermined areas of existence for the particles
5. Procedural Generation

Instantiating Game Objects

Random placement of objects

Procedural Material selection

Procedural Scale selection

Procedural Rotation selection

Placement and Distribution of objects

Hexadecimal Seeds

6. Mesh Displacements

- $\quad$ Affecting continuous surfaces

Changing appearance and geometry based on the relation to deformer game objects

7. Cameras

- Game view

- Visual effects

- $\quad$ Fields of View (FOV)

- Changing points of view

- $\quad$ Cameras as live textures 


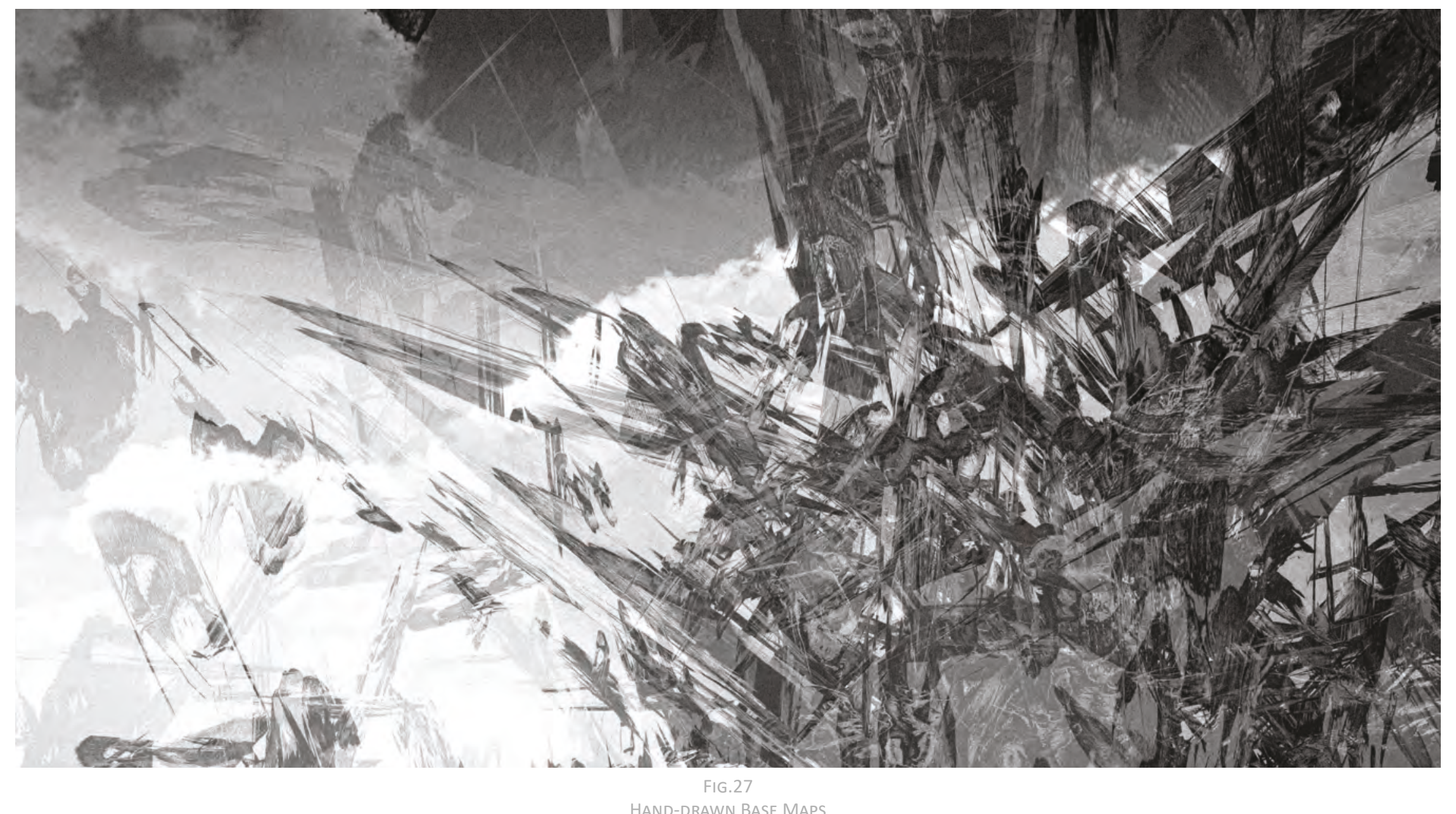

PENCIL DRAWINGS STRETCHED OVER BASIC MESH OBJECTS 


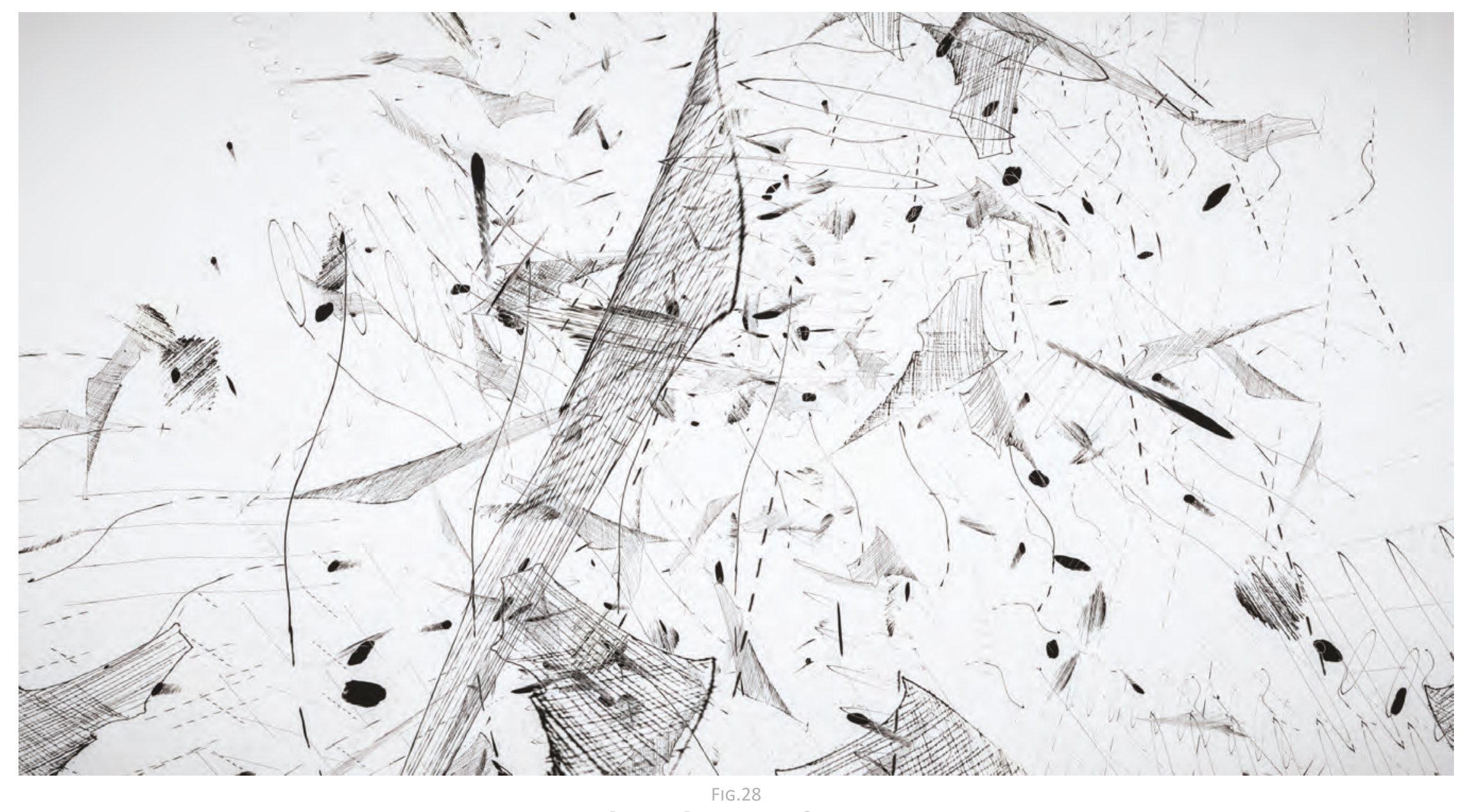

C\# SCRIPTS INSTANTIATE OBJECTS TEXTURED WITH HAND DRAWINGS PLACEING THEM WITHIN BOUNDARIES 


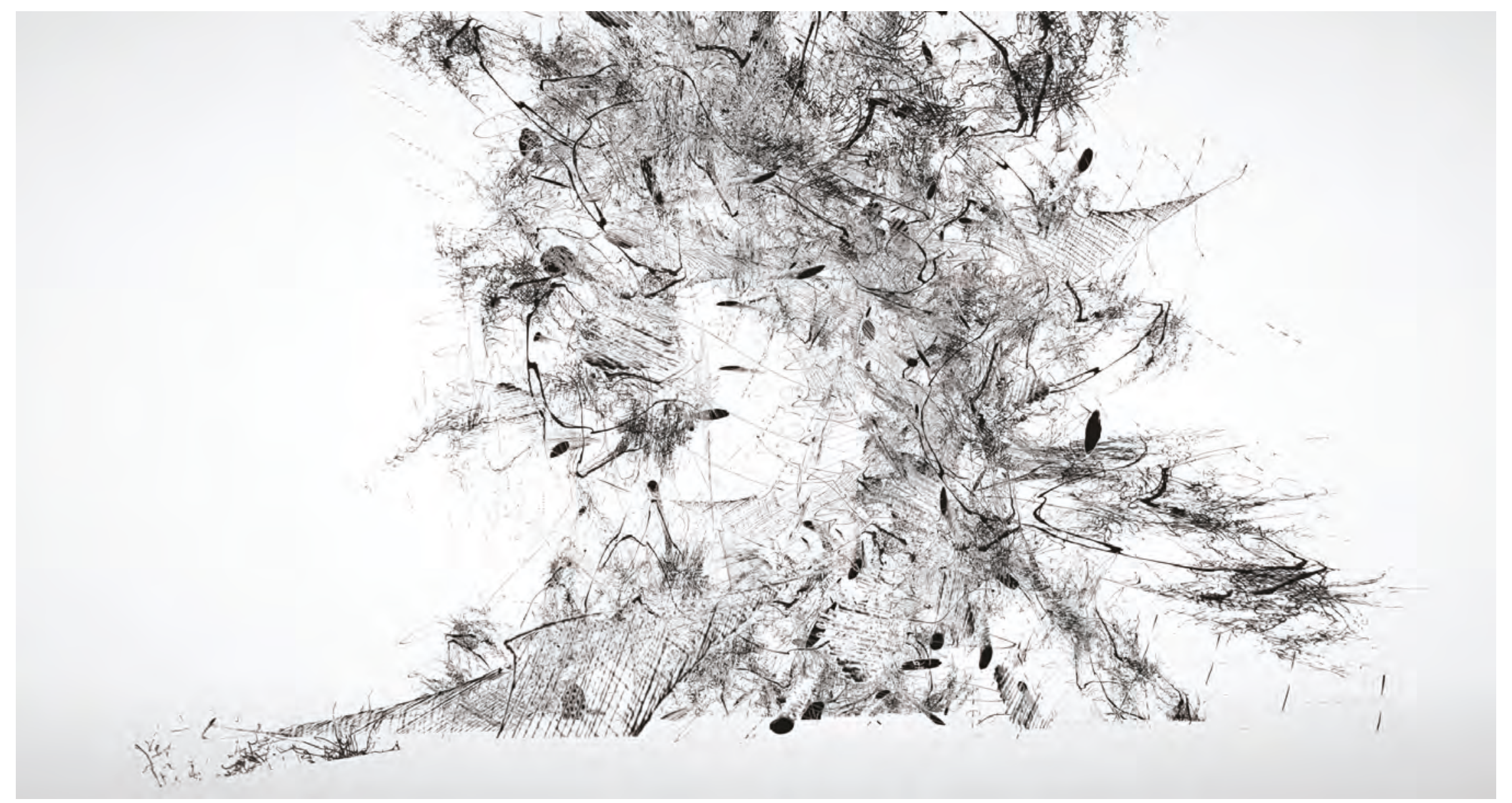

FIG.29

Procedural Scale, Rotation, Material Selections

CH SCRIPTS ROTATE, SCALE, AND SELECT DRAWINGS TO BE PLACED ON PLANES AND INSTANTIATED INTO THE GAMESPACE 


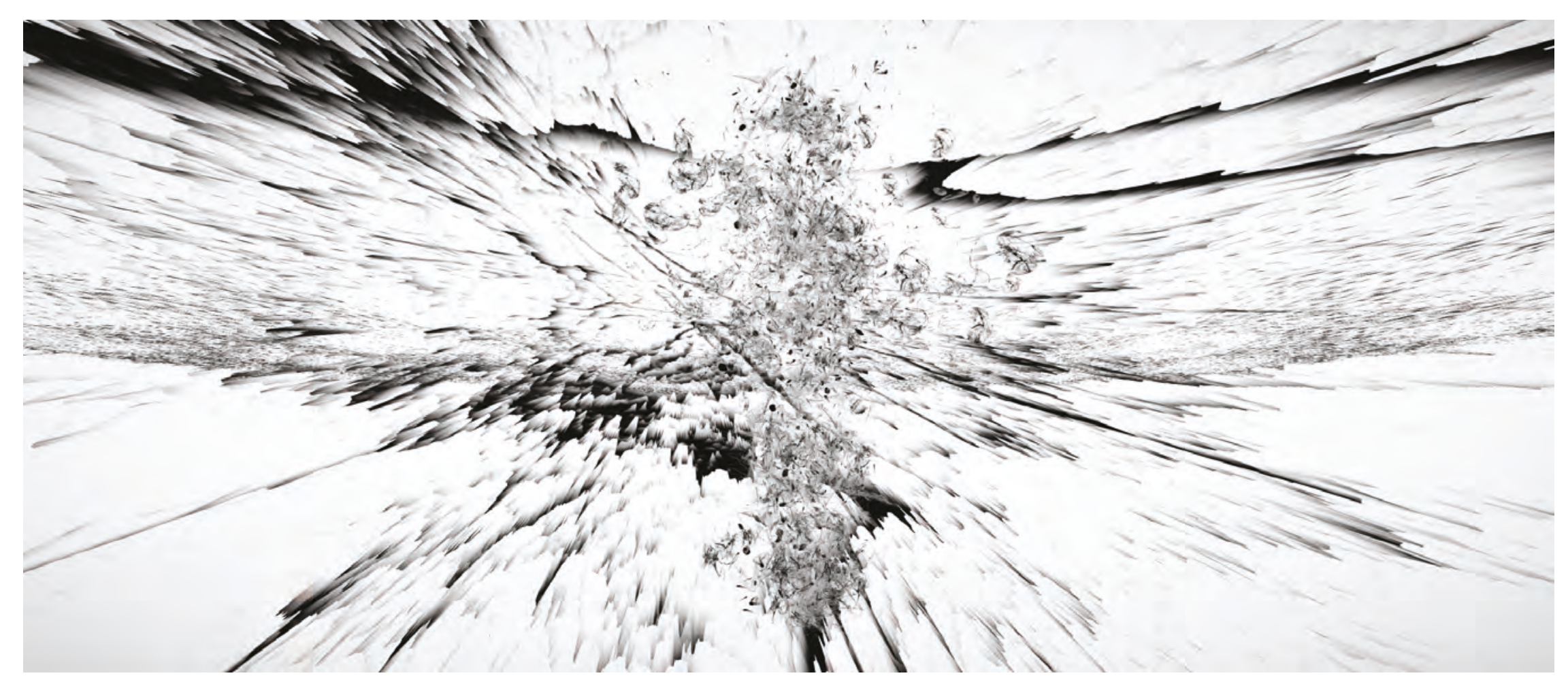

FIG.30

HeIght MAPS, VerTeX DISPLACEMENT, PARTICLE SYSTEMS

THE SURROUNDING 3-DIMENSIONAL GEOMETRY STARTS AS A FL AT SURFACE, WHERE THE PENCIL MARKS ARE DARKEST THE MESH OBJECTS ADD DEPTH. A PARTICLE SYSTEM CREATES AND DESTROYS MESH OBJECTS TEXTURED WITH PENCIL DRAWINGS 


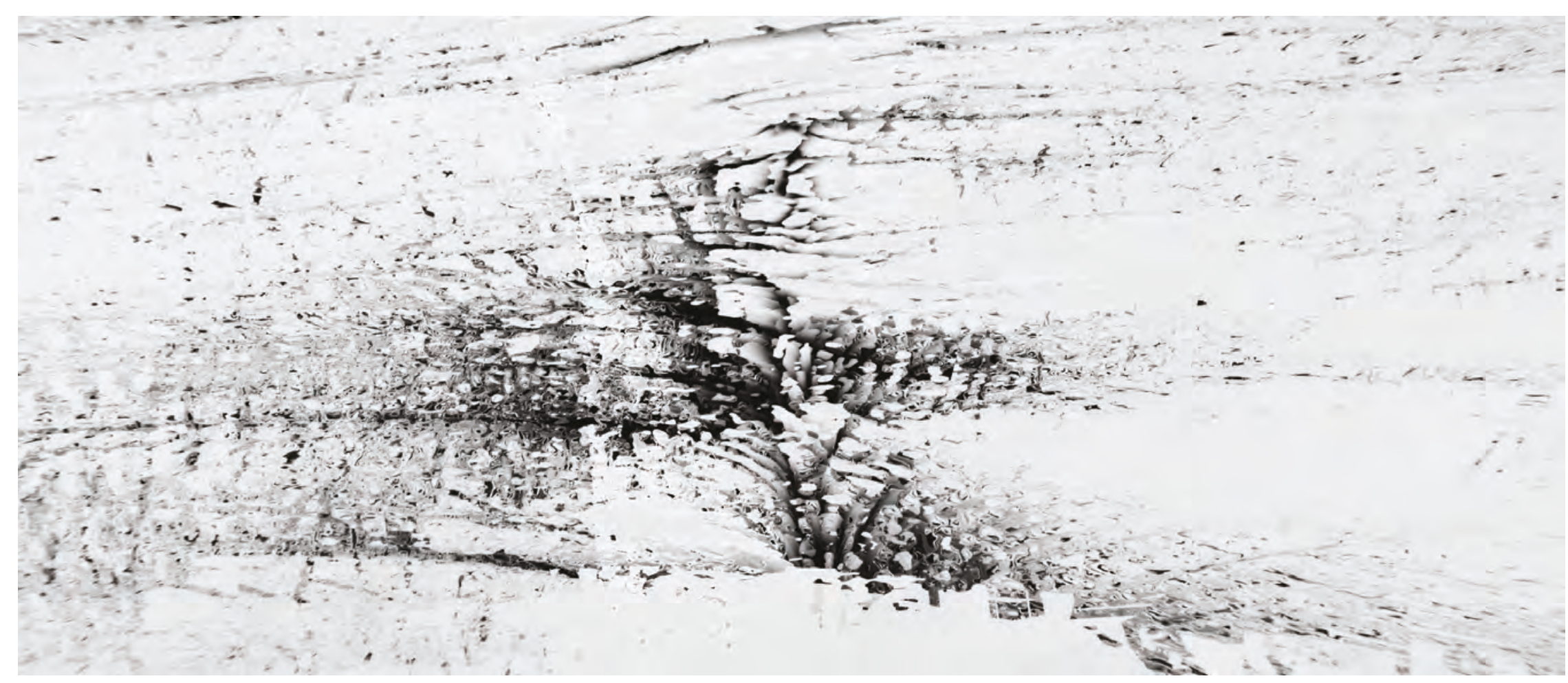

FIG.31

PIXEL DISPLACEMENT AND Field Of VIEW (FOV)

THE PENCIL MARKINGS SIT ON A 2-DIMENSIONAL PLANE BUT ARE RENDERED BY THE ENGINE AS HAVING DEPTH. THE FI ELD OF VIEW OF THE CHARACTER CAMERA DISTORTS AND ALTERS THE WORLD AROUND AN ASPHERICAL FI SH EYE LENS 


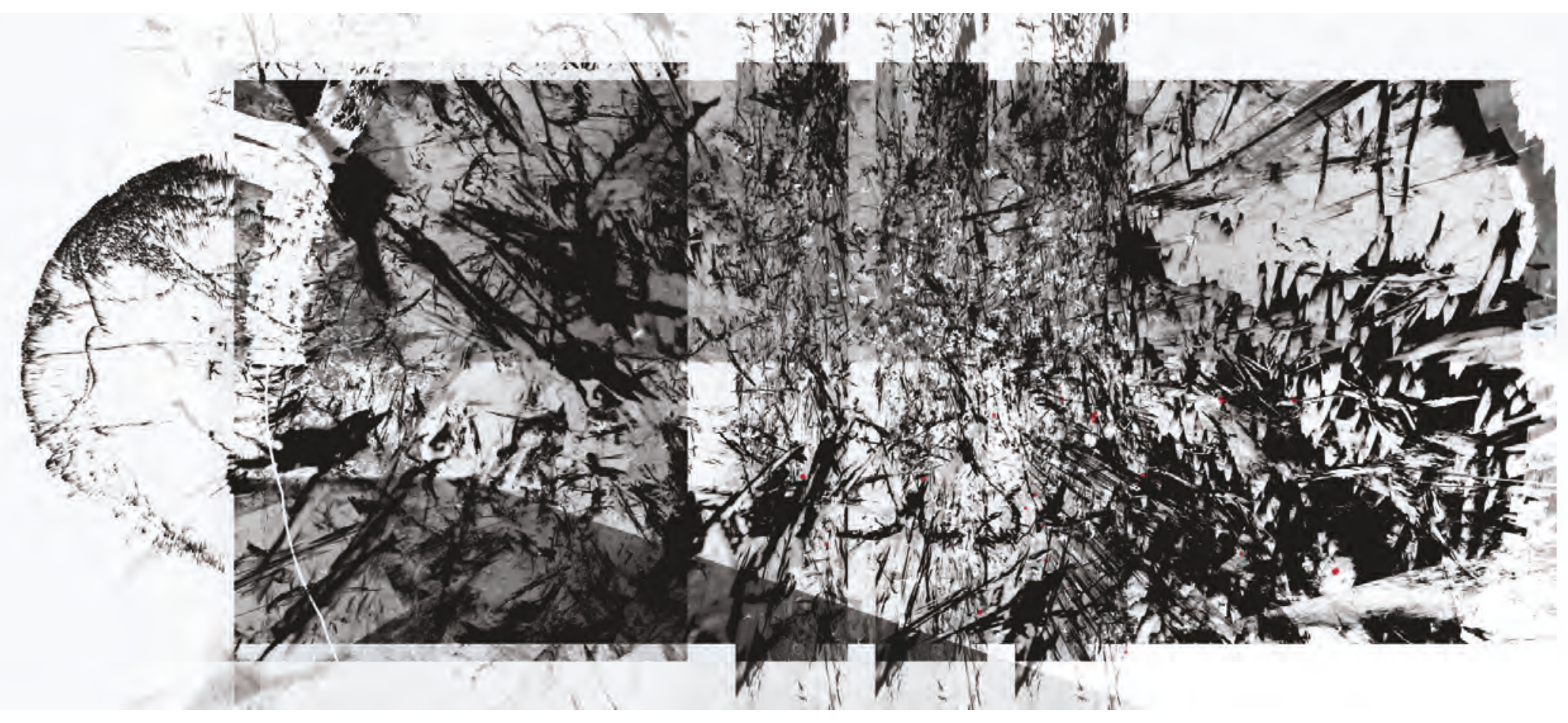

FIG.32

PHYSICS AND FORCE BASED INTERACTION WITH OBJECTS, MESH DISPLACEMENTS, DIRECTIONAL MOVEMENT

FORCED BASED INTERACTIONS AND MESH DISPLACEMENTS CAUSE THE TEXTURED PLANES TO BE FORCED AWAY FROM EACH OTHER. THE PLANES FALL UNDER THE ENGINES SIMULATED GRAVITY AND THE CHARACTER IS ABLE TO MOVE AND OBSERVE THESE PLANES 


\section{Research Through Drawing: Modelling and Unity Scripts}

Previously only 2-dimensional, the hand drawings now existed in a 3-dimensional virtual reality. It was now possible to observe these marking in space rather than on a plane. The drawings became allegory for my coded algorithms. Mackenzie Wark has written extensively on the connection between the algorithm and its representation in their book Gamer Theory. This is described by the term allegorithm. They define the concept of allegorithm as "The gamer selects one sequence after another, and gradually learns what they do- that's algorithm. The gamer discovers a relationship between appearances and algorithm in the game, which is a double of the relation between appearances and a putative algorithm in gamespace- that's allegorithm." (Wark 2007, 31) My drawings functioned as allegorithms allowing the player to relate their appearances to the code that created them. One step further it allowed the player character to influence and change the representations in game space. 


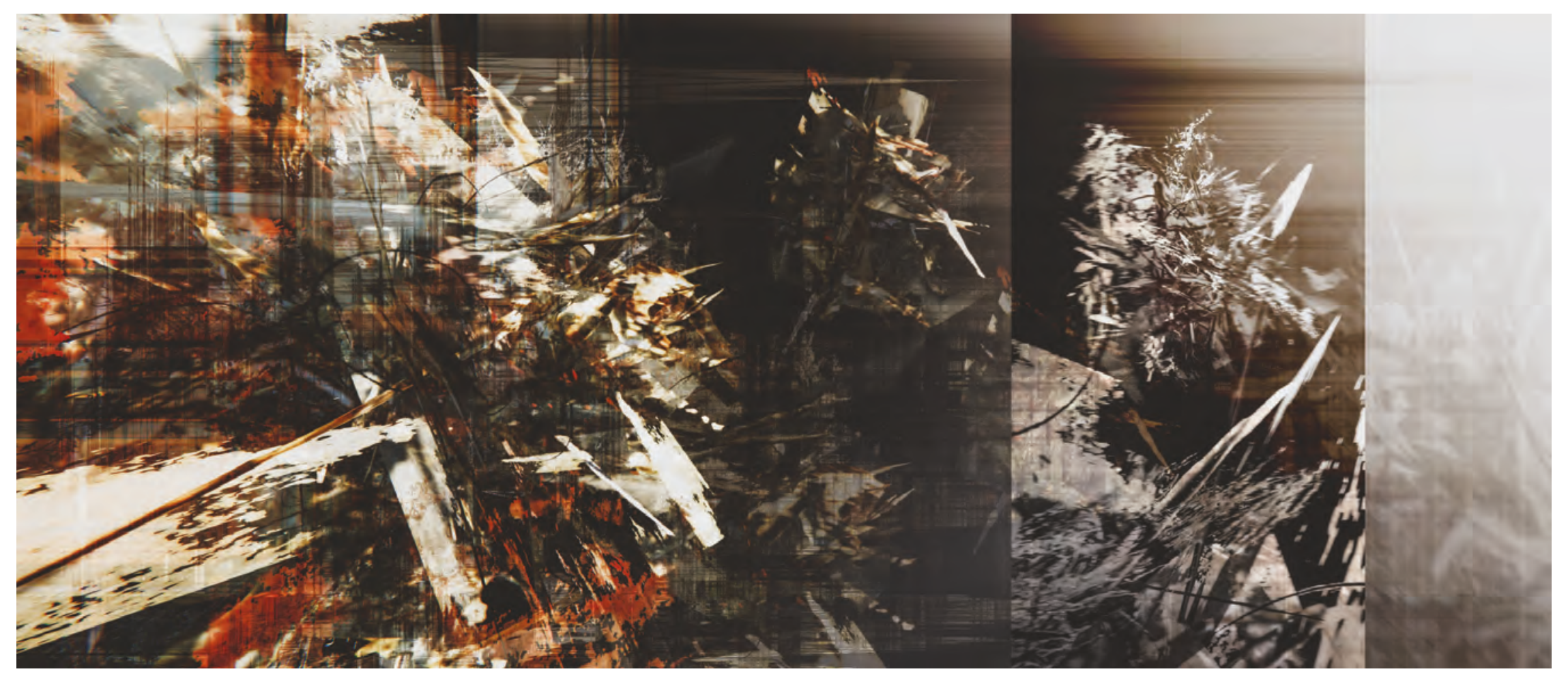

fig. 33 Perspective and elevation collage 


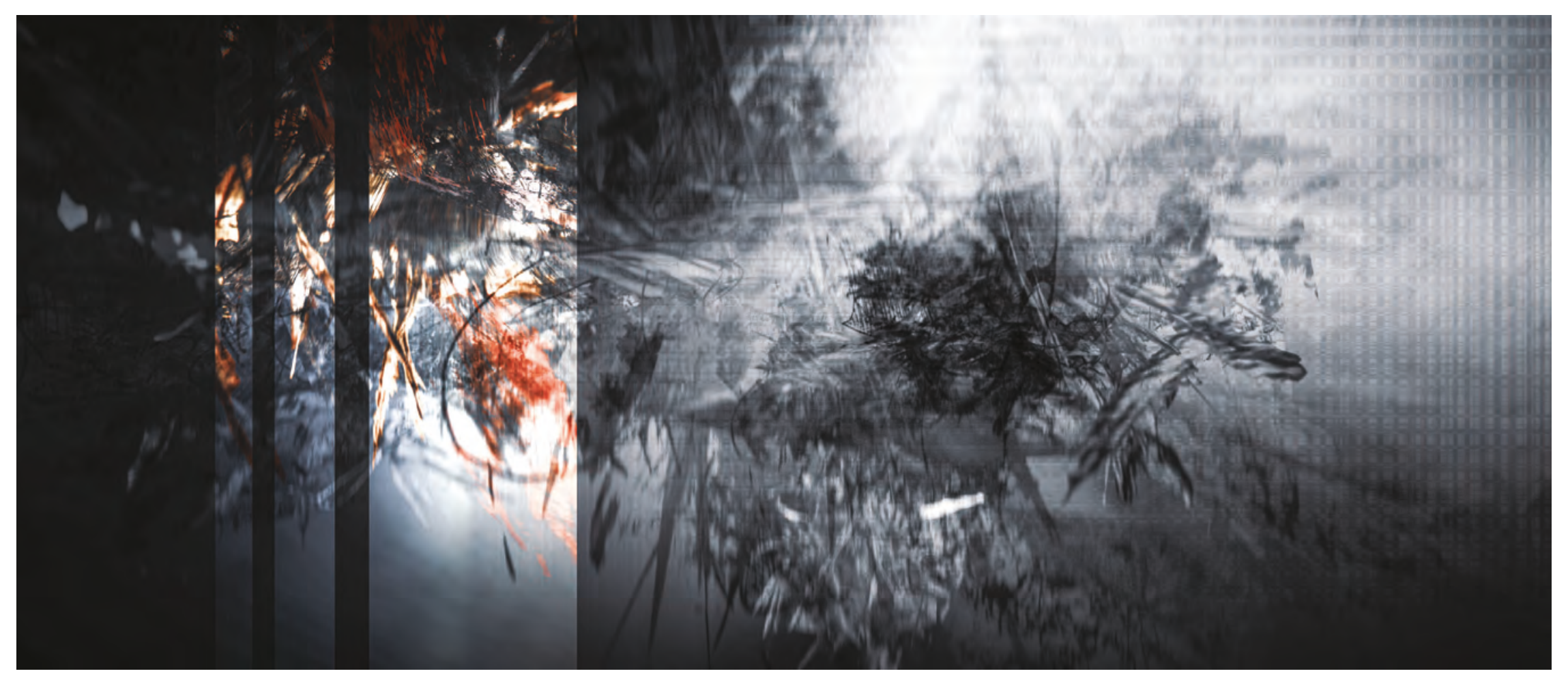

FIG. 34 PERSPECTIVE ANd SECTION COLLAgE 


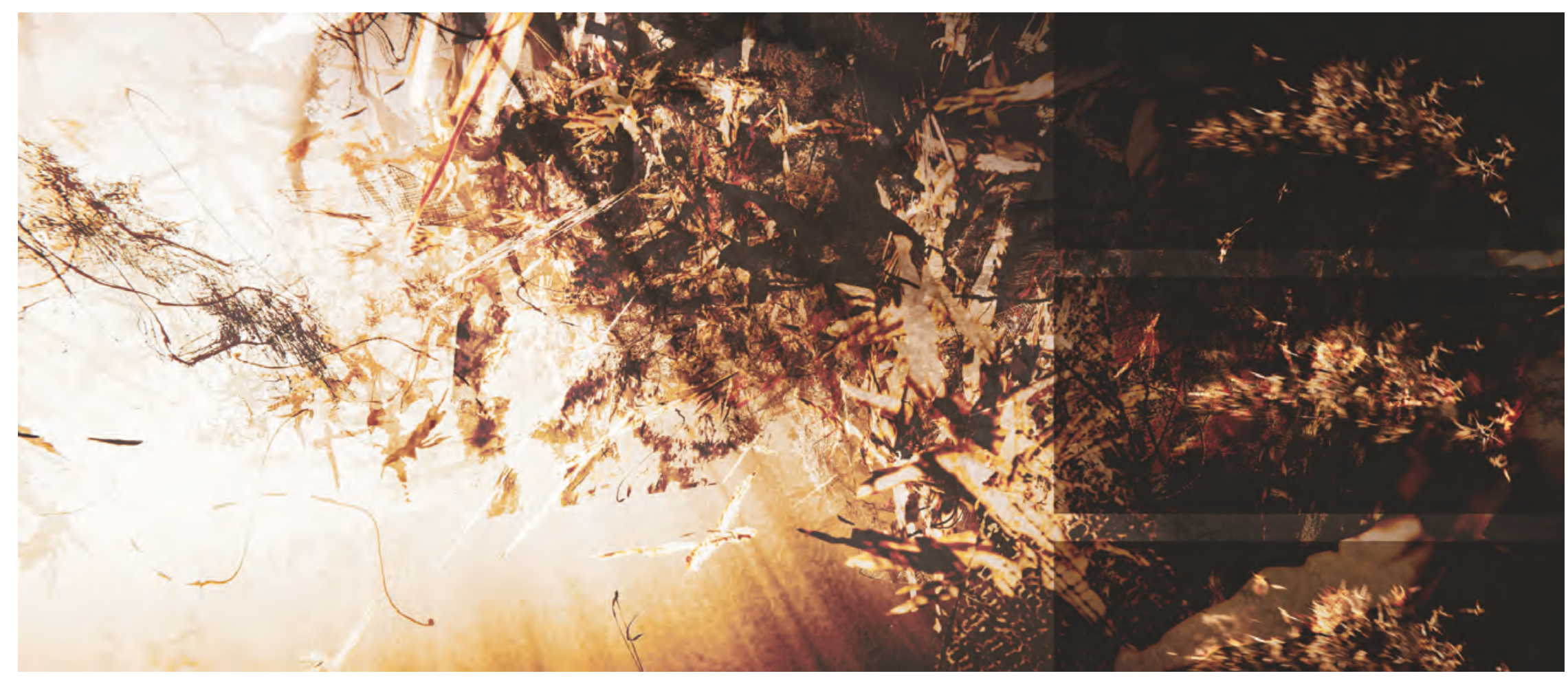

fig. 35 Perspective and plan collage 


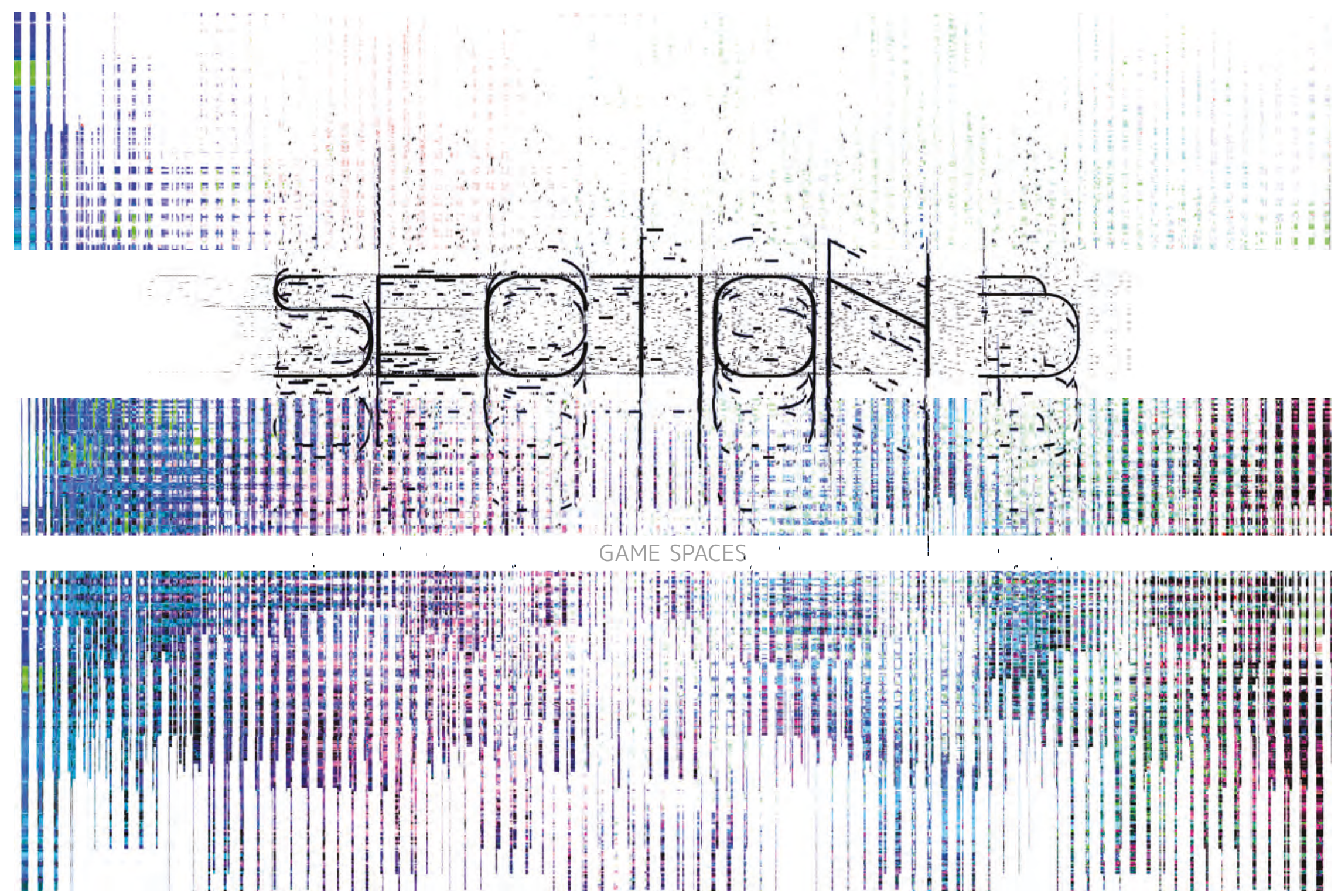




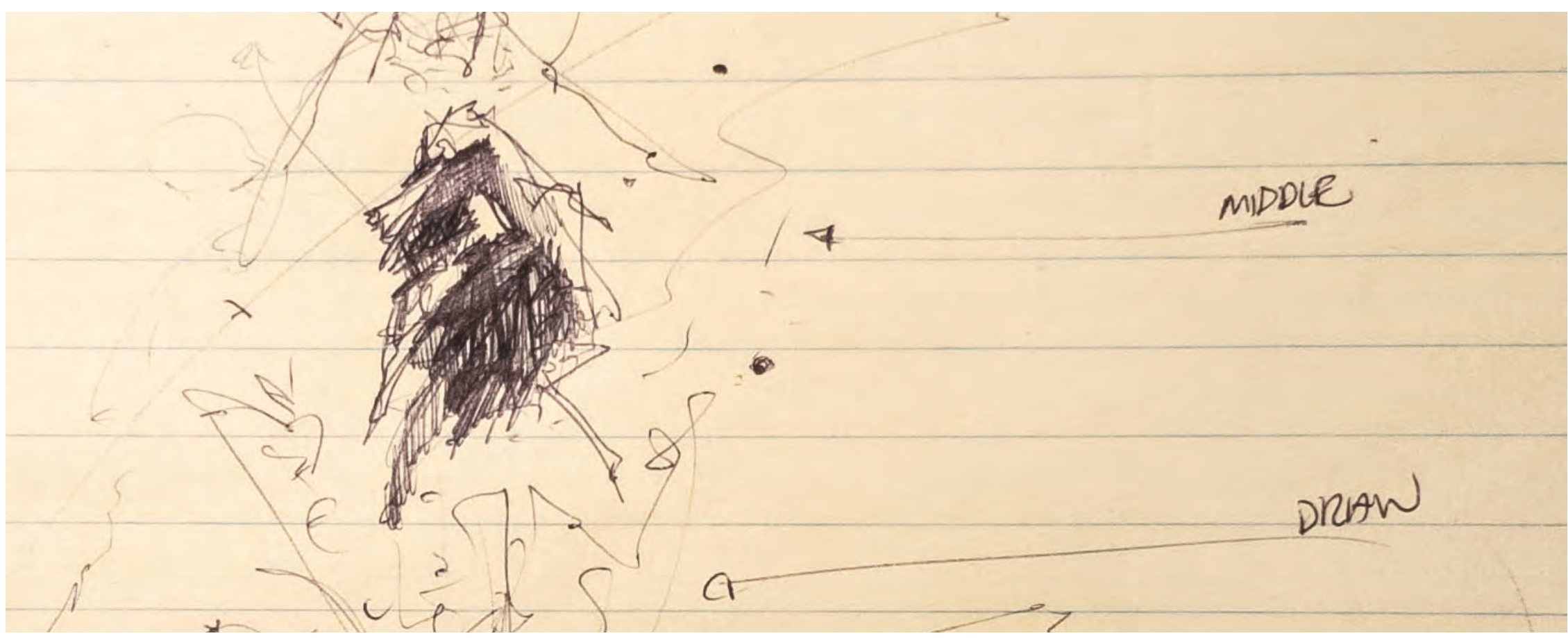

DeVeloping the GAME

To reconsider the relationship between architecture and game space I sought to develop a game that gave the player agency to manipulate space in unforeseeable ways. The skill which was developed over the course of the first half of the project could now be creatively implemented. The aim of the implementation was to create a series of levels that would allow the player the ability to play in these levels. To do this, I conceptualized the game as a series of spaces in which objects could manipulate architectural forms as well as natural forms. The following is an explanation of the various ways that this spatial manipulation was conceived and the resultant spatial representations that they generated.

FIG. 36 DESIGN SKETCH ON YELLOW PAD 


\section{Object of Interaction: The Sphere}

As the ball rolls the world rotates around it. When I connected virtual geometries to the sphere, I began to play for the first time. The player character has only two ways to interact with game objects, to pick up objects and to throw objects combined with the character's movement. When the character picks up an object and puts it down the interaction ends. With the sphere the difference is that it retains some motion when it is dropped or thrown. The games physics engine maintains the movement of the object as it would it in the real world. When the motion, position, and rotation of the sphere is connected to the shape of the virtual environment the results change drastically with each interaction. The shape of the sphere remains motionless when it is undisturbed at the beginning of each level. Once disturbed the effects on the geometry can be seen deforming in relation to the sphere's movement. With minimum friction applied to the materials that the sphere interacts with; it remains in motion for a considerable period after each interaction. Even a minor force applied to the sphere, intentional or accidental, can result in drastic changes to the level due to rotational or directional deformations of level's form. The fun in every level starts when the player begins shaping the world with the sphere.

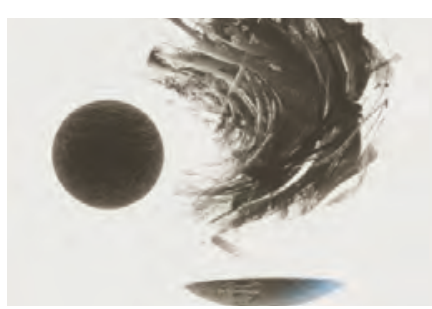

FIG. 37 LEVEL 1 INTERACTABLE SPHERE

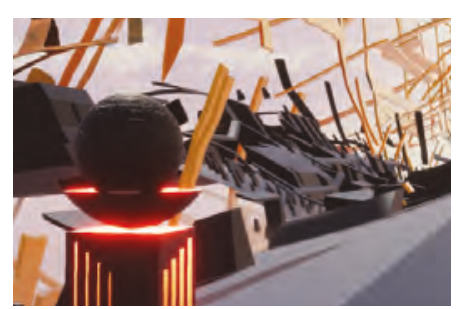

FIG. 38 LEVEL 2 INTERACTABLE SPHERE

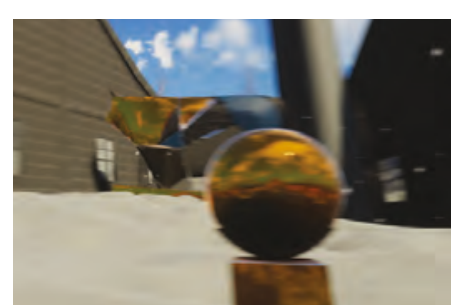

FIG. 39 LEVEL 3 INTERACTABLE SPHERE 
LeVEL 1

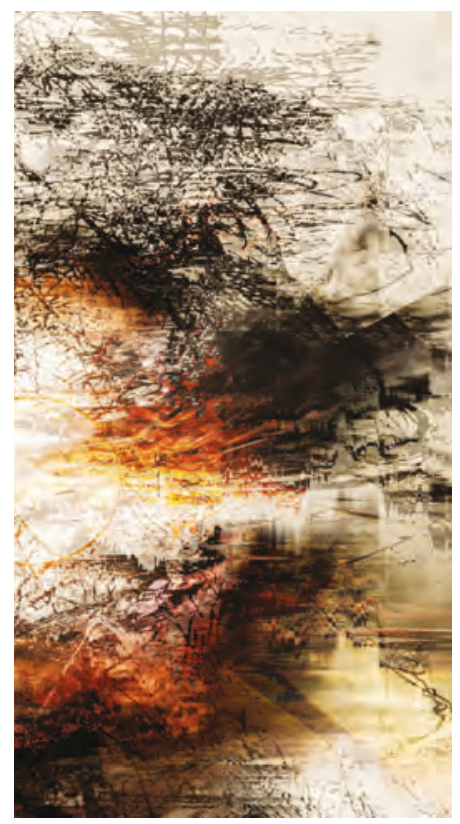

INTERACTING WITH THE

2-DIMENSIONAL
LeVel 2

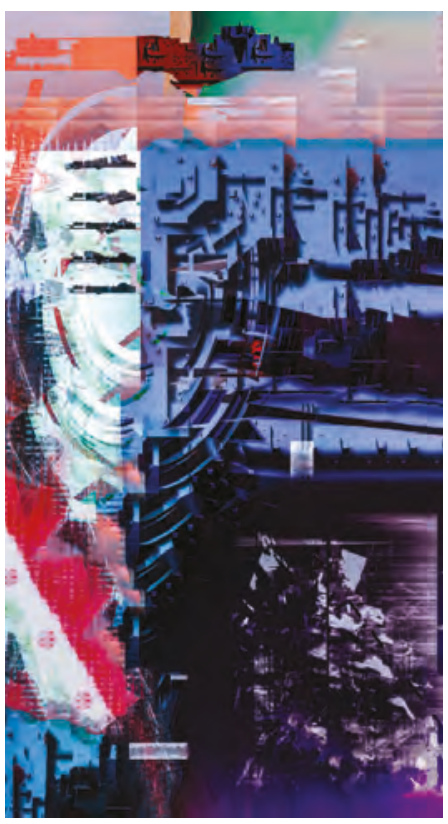

INTERACTING WITH THE

3-DIMENSIONAL
LEVEL 3

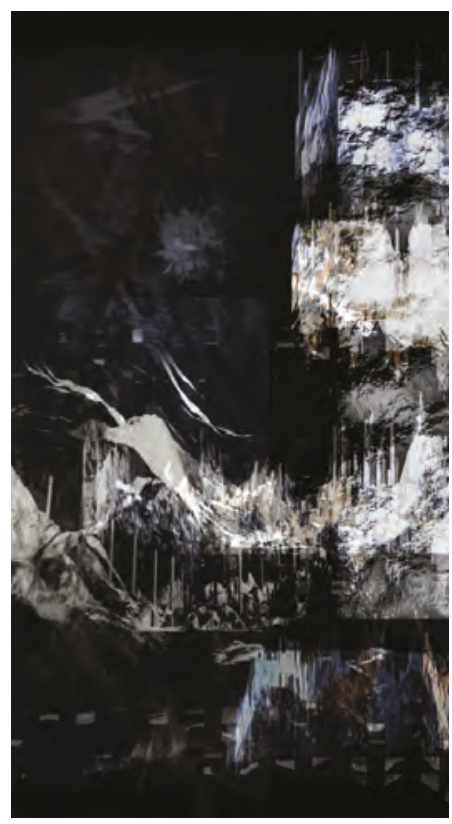

EXPERIENCING VIRTUAL SPATIAL

POSSIBILITIES

LeVel Design and Experience

The focus was creating short experiences at varied scales: a small-scale room, a medium-scale architectural space, and a large-scale natural environment. Each space features an experience that is completed in a short period of time that familiarizes the player with the environment. Each level is then to be replayed to explore the variations that can occur in the spatial experience. 
Level Concept Graphite Drawing

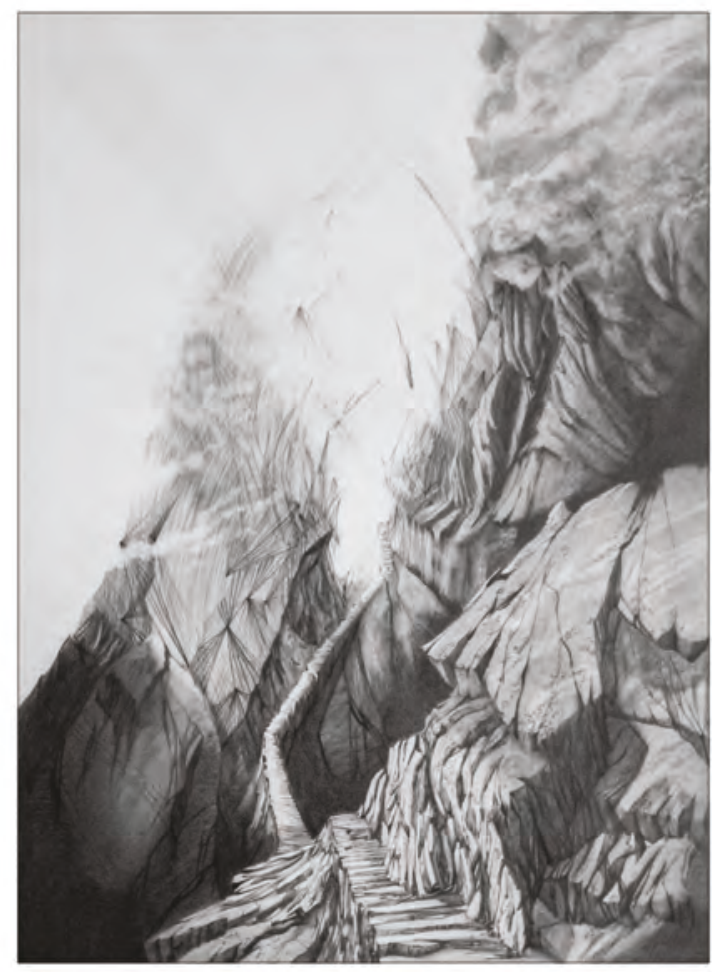

EIG. 40 EARIY CONCEPT DRAWING FOR THE LEVEL 3 ENVIRONMENT WITH ADIACENT DETAIL

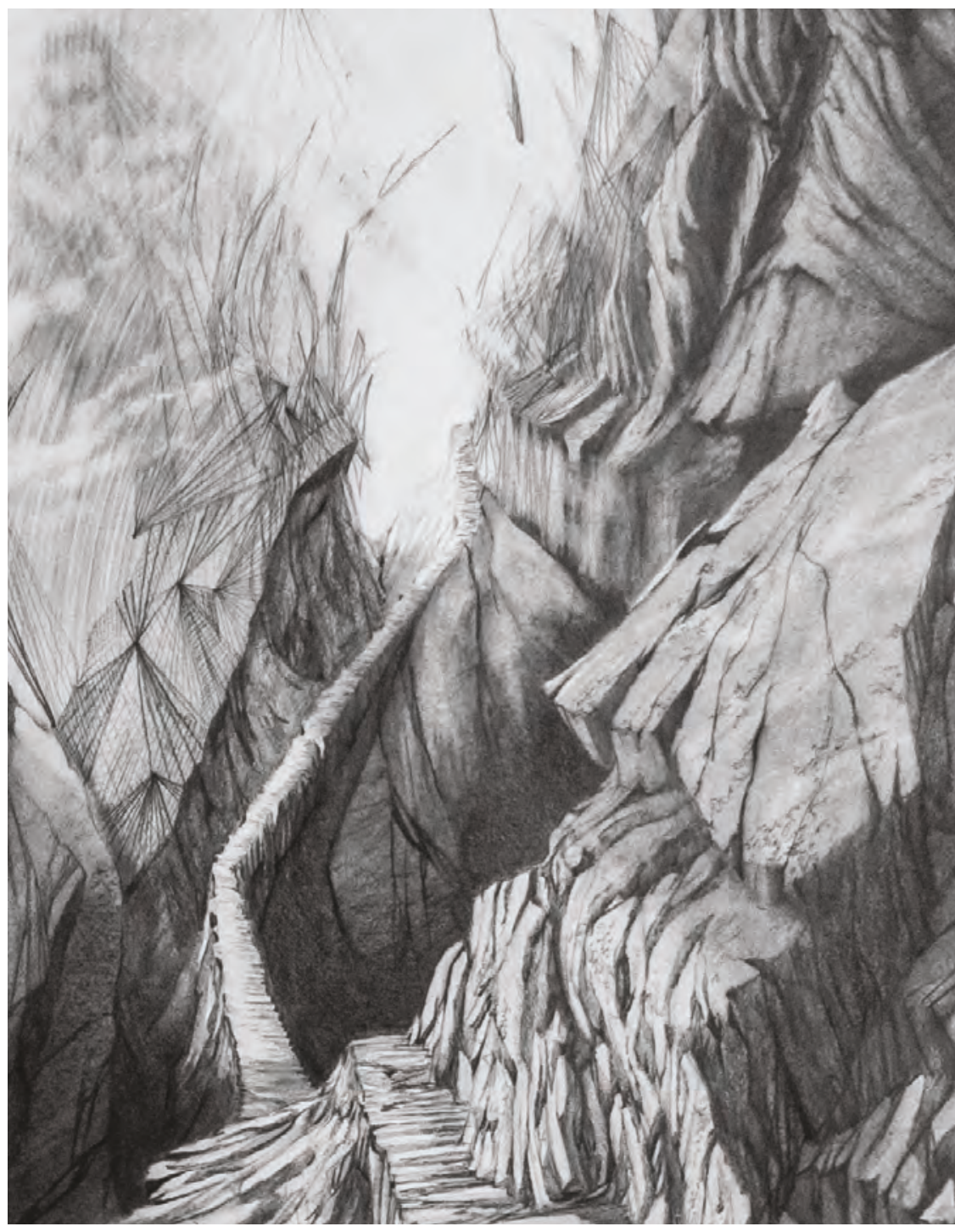

50 

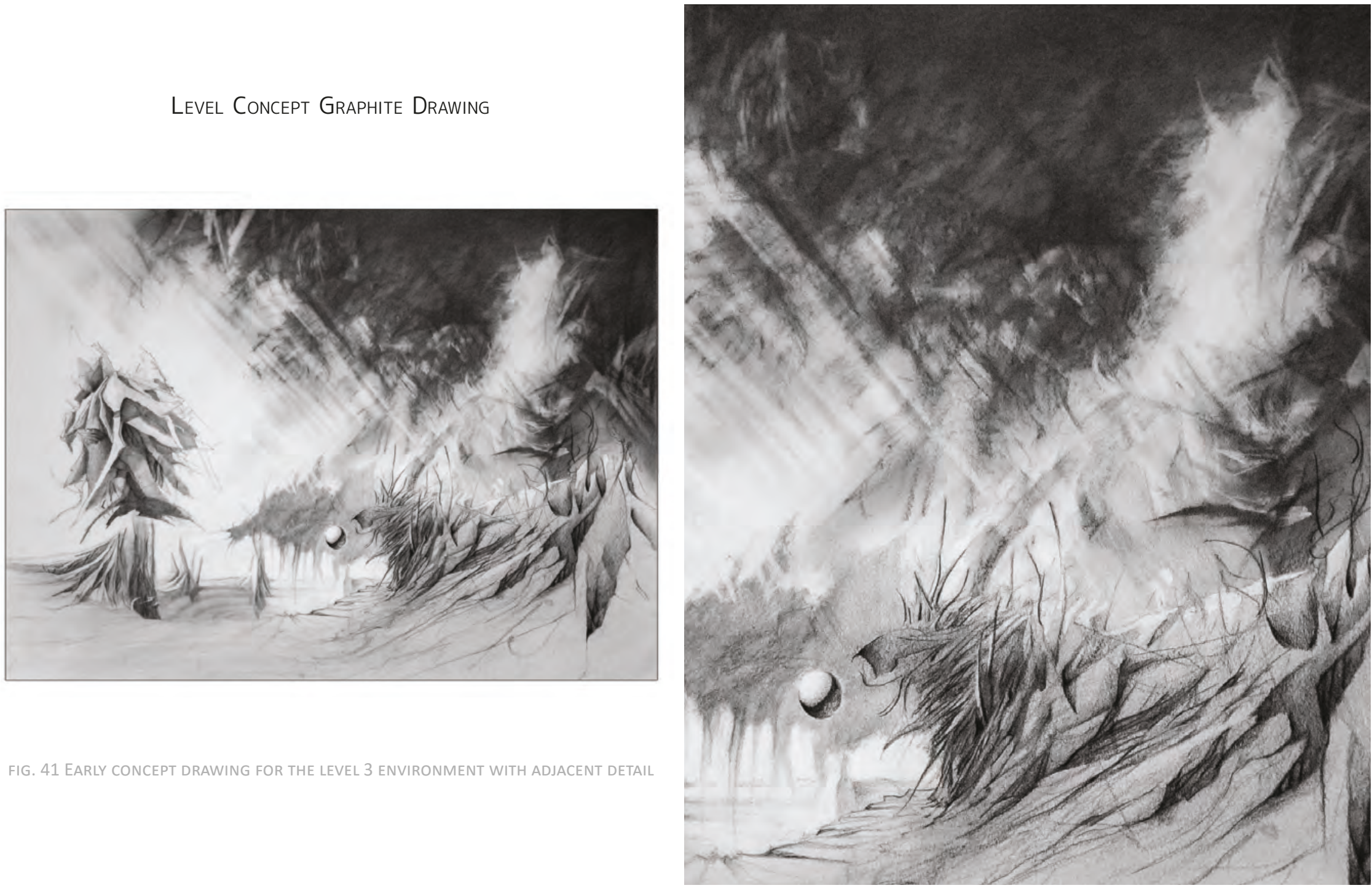

FIG. 41 EARLY CONCEPT DRAWING FOR THE LEVEL 3 ENVIRONMENT WITH ADJACENT DETAIL 
LEVEL 1

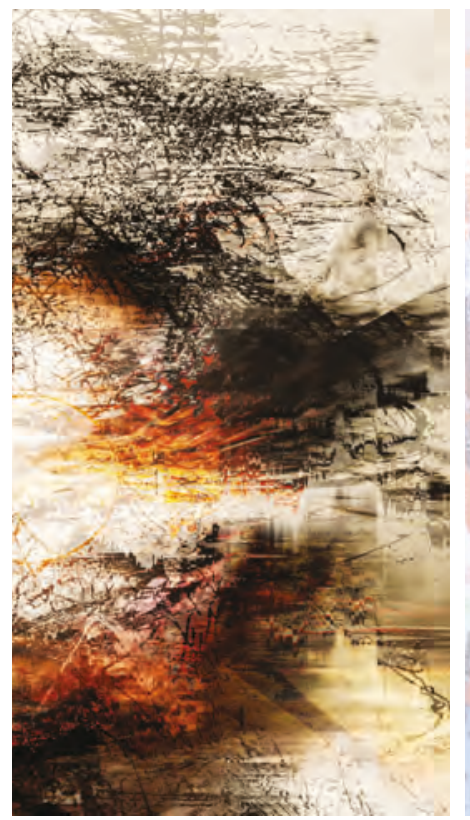

INTERACTING WITH THE

2-DIMENSIONAL
LEVEL 2

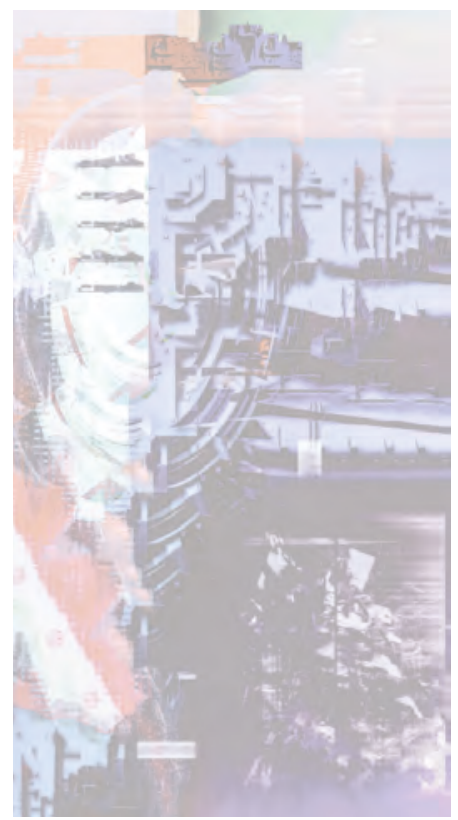

3-DIMENSIONAI
LEVEL 3

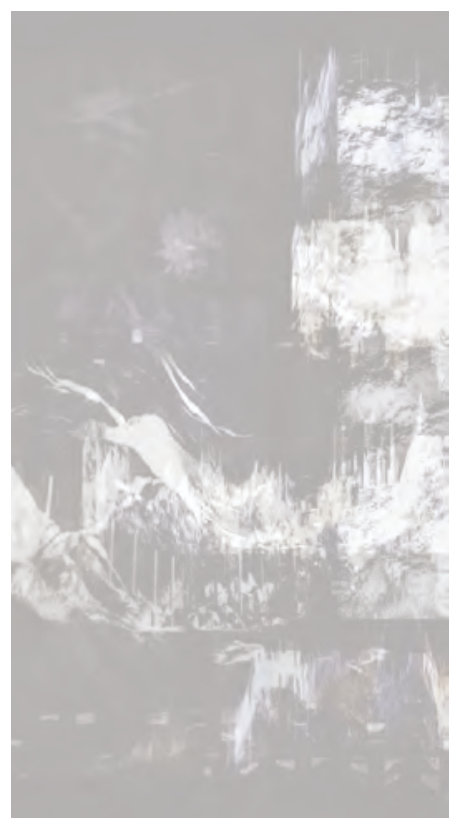

OSSIBILITIES 


\section{LeVel 1}

The first level is designed as an intimate encounter with hand drawings in a game space. Here the player and character will acquaint themselves with one another in a space that is not directly affected by player action. The experience is designed to allow for distortion and glitching of the hand drawings in the space but not the space itself. The room's plan is a square 100 units by 100 units with a large cauldron in the centre. The floor of the room is a 2-dimensional plane textured with an early ink study. This gestural ink drawing uses pixel displacement mapping. This setting renders the black areas of ink as having depth on a 2-dimensional plane. Floating above the cauldron are one hundred 2-dimensional planes textured with graphite. Scattered around the floor are light emitting spheres that the player character can interact with. Each sphere is coded to manipulate the suspended drawing based on their relation to the spheres. Above the cauldron are objects called boids. Boids are a simple form of artificial intelligence that are used to simulate the behaviour of birds or other animals that move in groups. In the game space they emit light and fly around the cauldron. The boids are basic Al objects. The boids seek out the same spheres that the character is meant to move. The boids are included in order to encourage the player to seek the sphere to engage with the drawings. In later levels the boids are referenced again as objects that do not normally behave as animals. The experience when I play the level is described as follows.

I begin in a small box. Before me are drawings, floating above a large crucible. Spheres litter the ground, each giving off an intense warmth. Looking down at them I notice the plane I am standing on is both ultrathin and transparent. The floor is split by dark cracks that extend beyond my vision's limits. The lines that split the floor are made by the same hand as the drawings above me. Following the other entities that flock around the space I approach one of the spheres. When I pick up the sphere the drawings spasm. I throw the ball into the crucible which causes the planes to erupt twisting away from the center of the room. A swarm of dark boids fly over my head searching for the remaining spheres they consume as food. I lose sight of the small flying objects as the drawings reach a point where they can no longer be rendered properly within the game's reality. The glitches break the space unable to be rendered anymore. 
REPRESENTATION

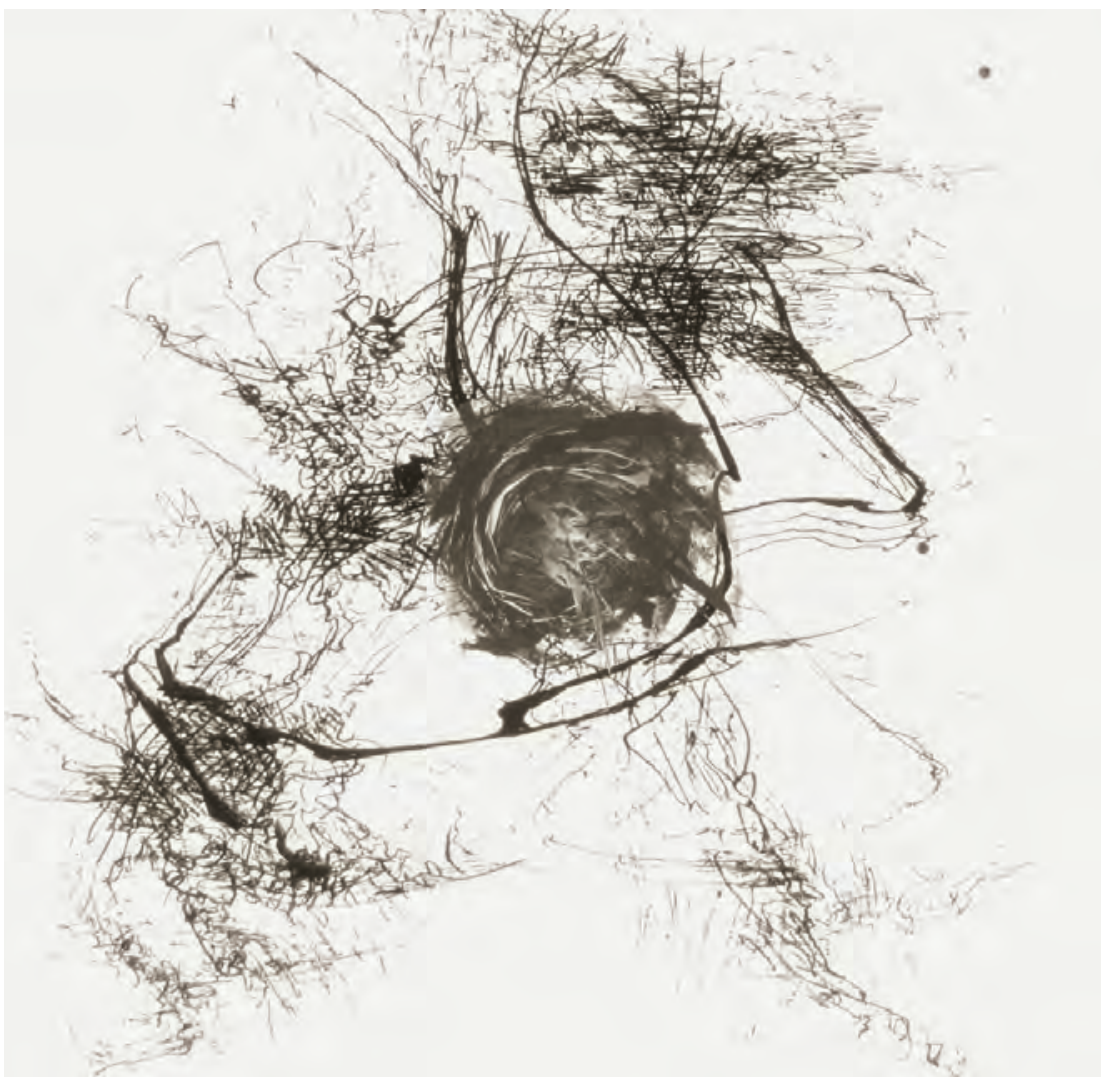

EXPERIENCE

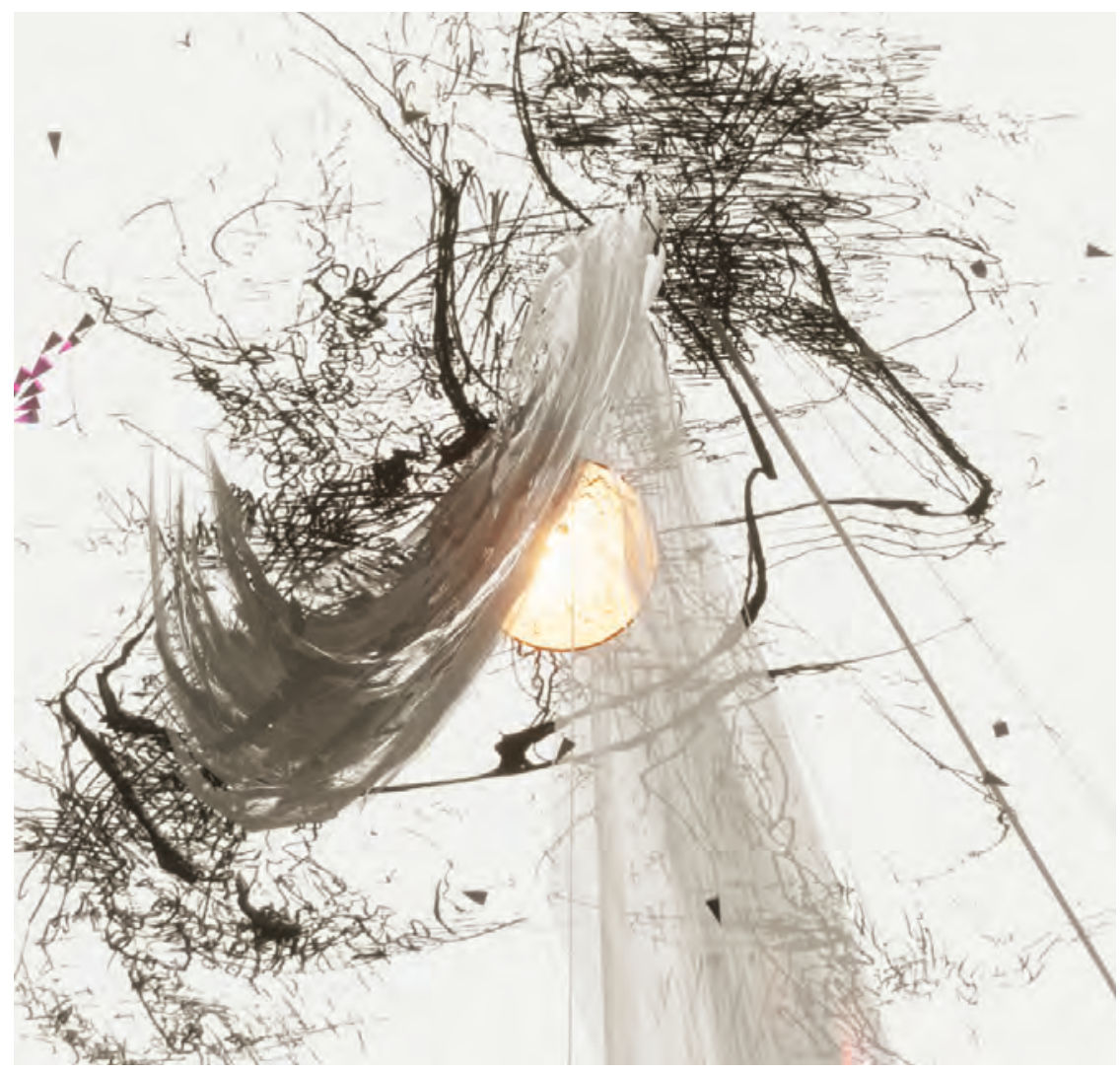


REPRESENTATION

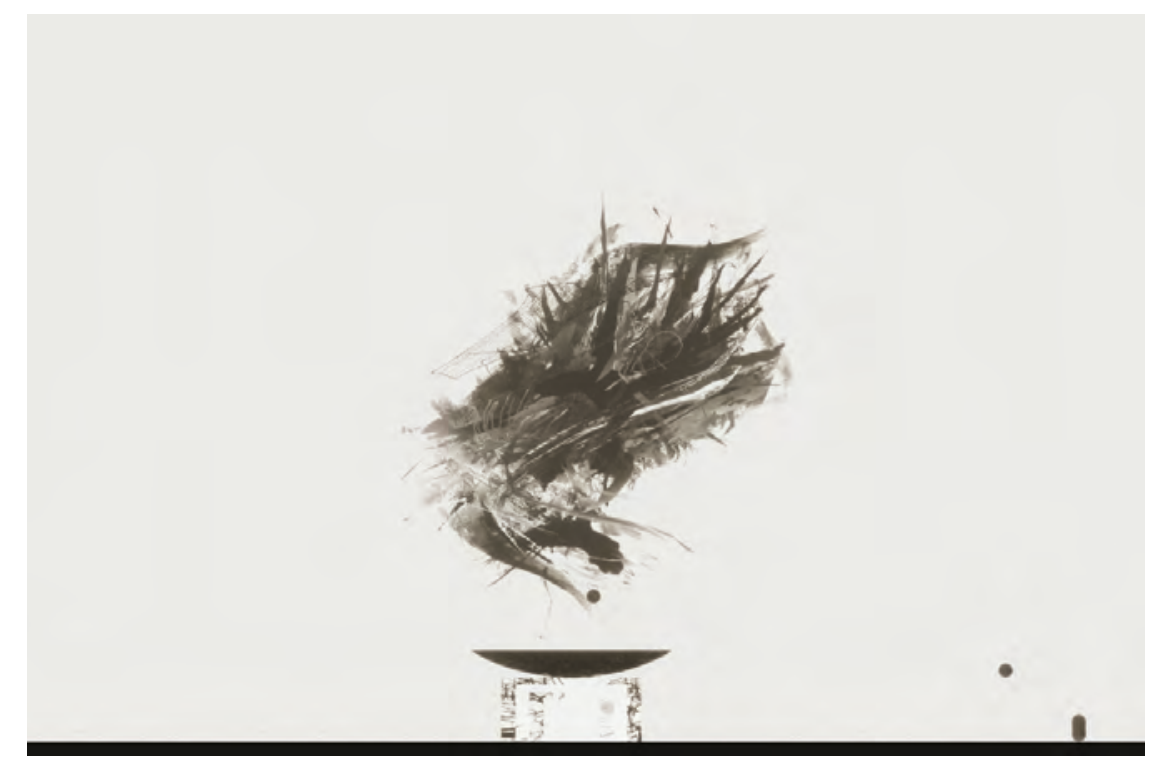

FIG. 44 SECTION - COLLECTION OF HAND DRAWINGS TEXTURED ON 2-DIMENSIONAL PLANES DORMANT ABOVE THE CAULDRON WITH THE PLAYER AND THE SPHERE CURRENTLY UNNAFFECTED BY GRAVITY
EXPERIENCE

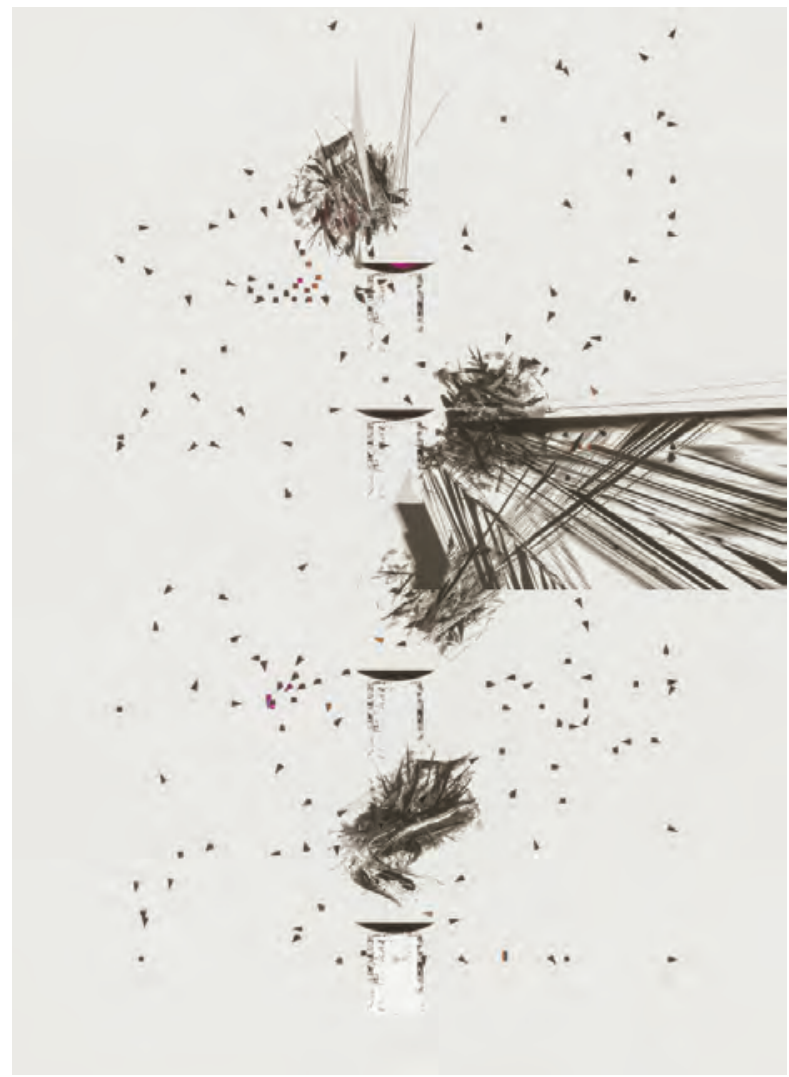

FIG. 45 SECTIONS - SERIES OF SECTIONS AS THE PLAYER INTERACTS WITH THE DIFFERENT SPHERES, CAUSING DEFORMATIONS AND GLITCHES IN THE COLLECTION OF DRAWINGS. BOIDS CAN BE SEEN FLYING. 
RePRESENTATION

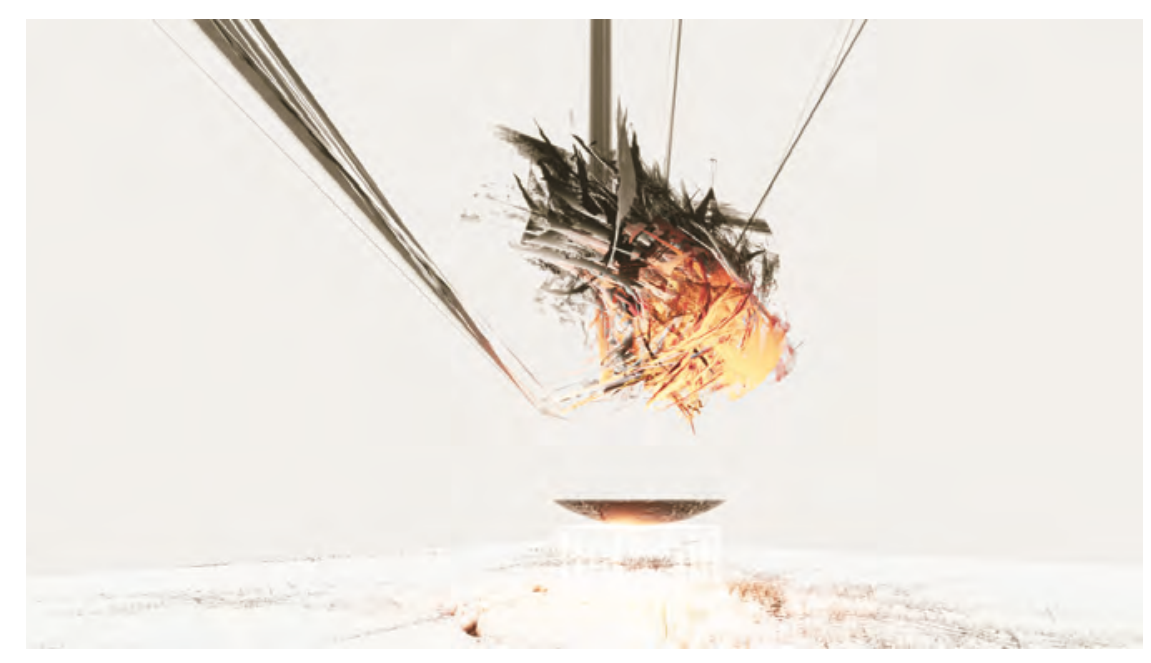
SPHERES
EXPERIENCE

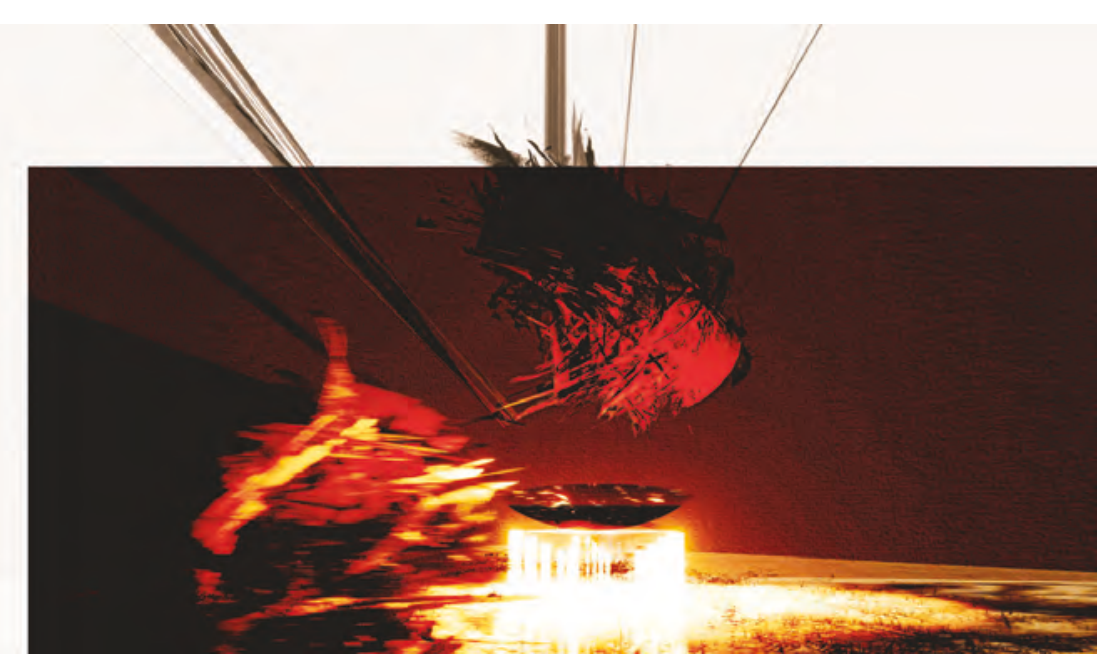

FIG. 47 OBJECT - TWISTS AND GLITCHES AS THE SPHERES ARE THROWN BY THE PLAYER 
REPRESENTATION

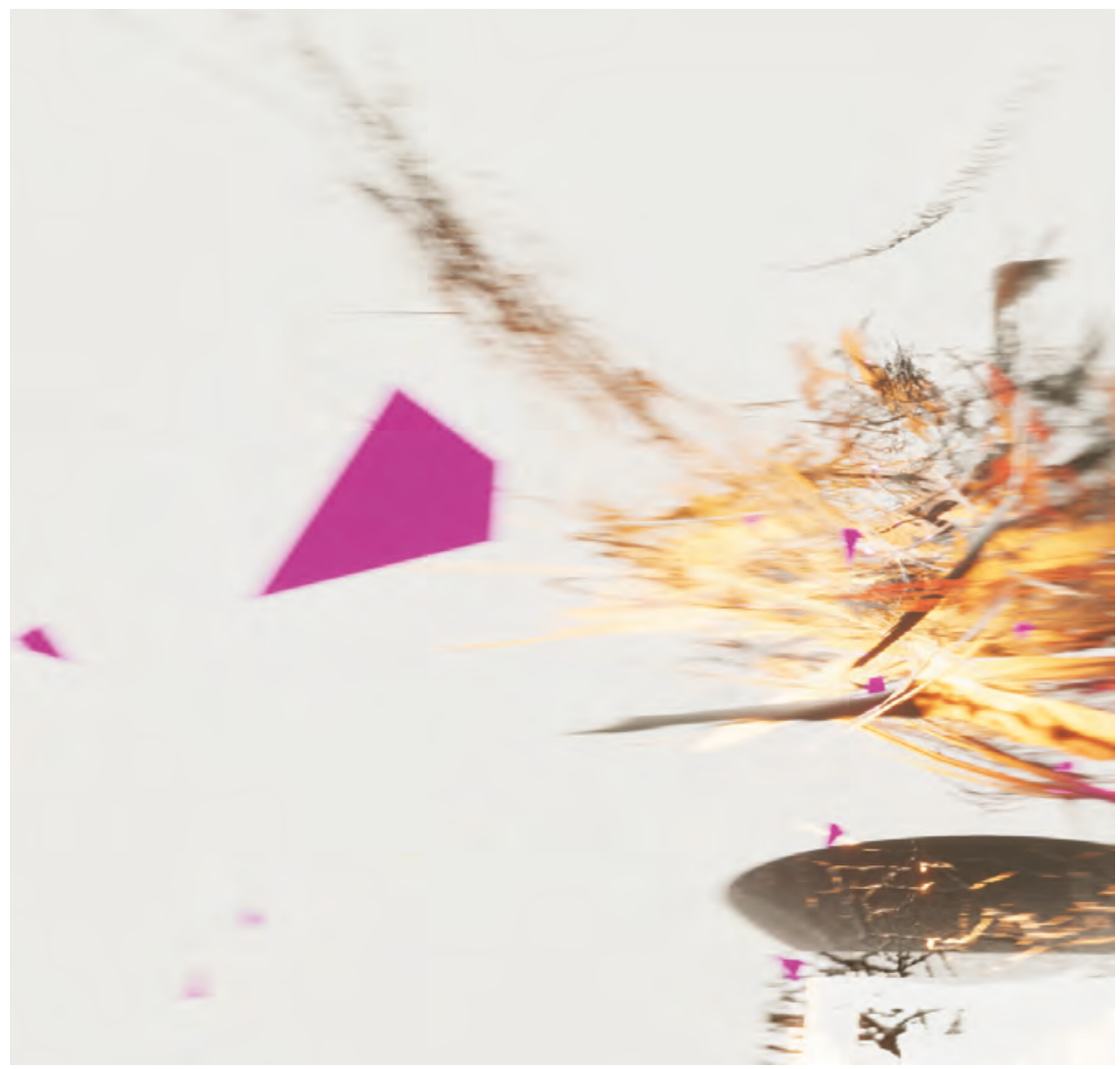

FIG. 48 AI BOIDS - SIMPLE GEOMETRIES OR PYRAMIDS IN THIS CASE ARE THE STANDARD FORI THAT BOIDS TAKE WHEN BEING DEVELOPED

\section{EXPERIENCE}

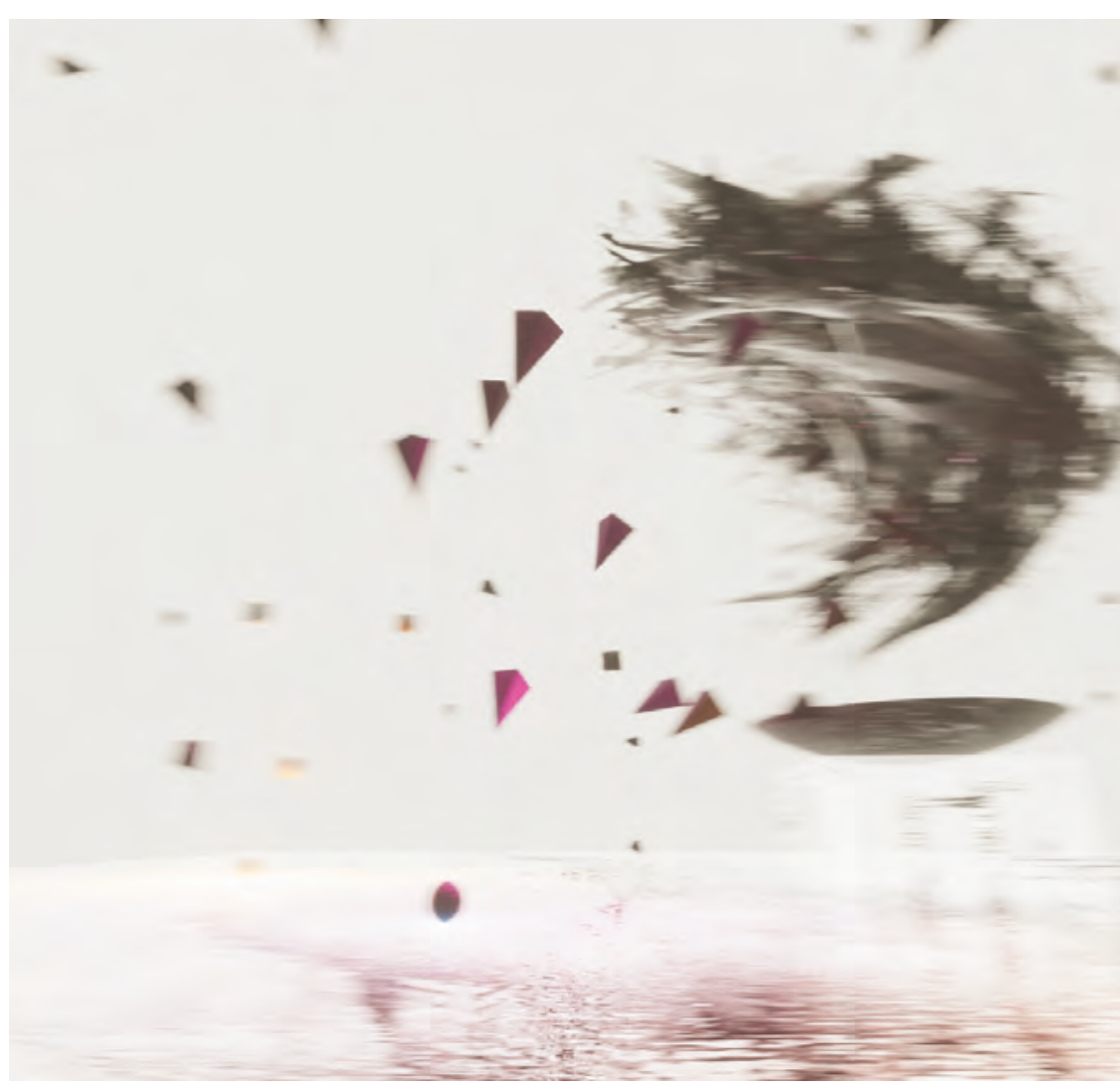

FIG. 49 AI BOIDS - BASED ON THE MOVEMENT OF SCHOOLS OF FISH OR FLOCKS OF BIRDS THE BOIDS MOVE, AVOID, FOLLOW, AND FEED ON VIRTUAL OBJECTS 
Representation

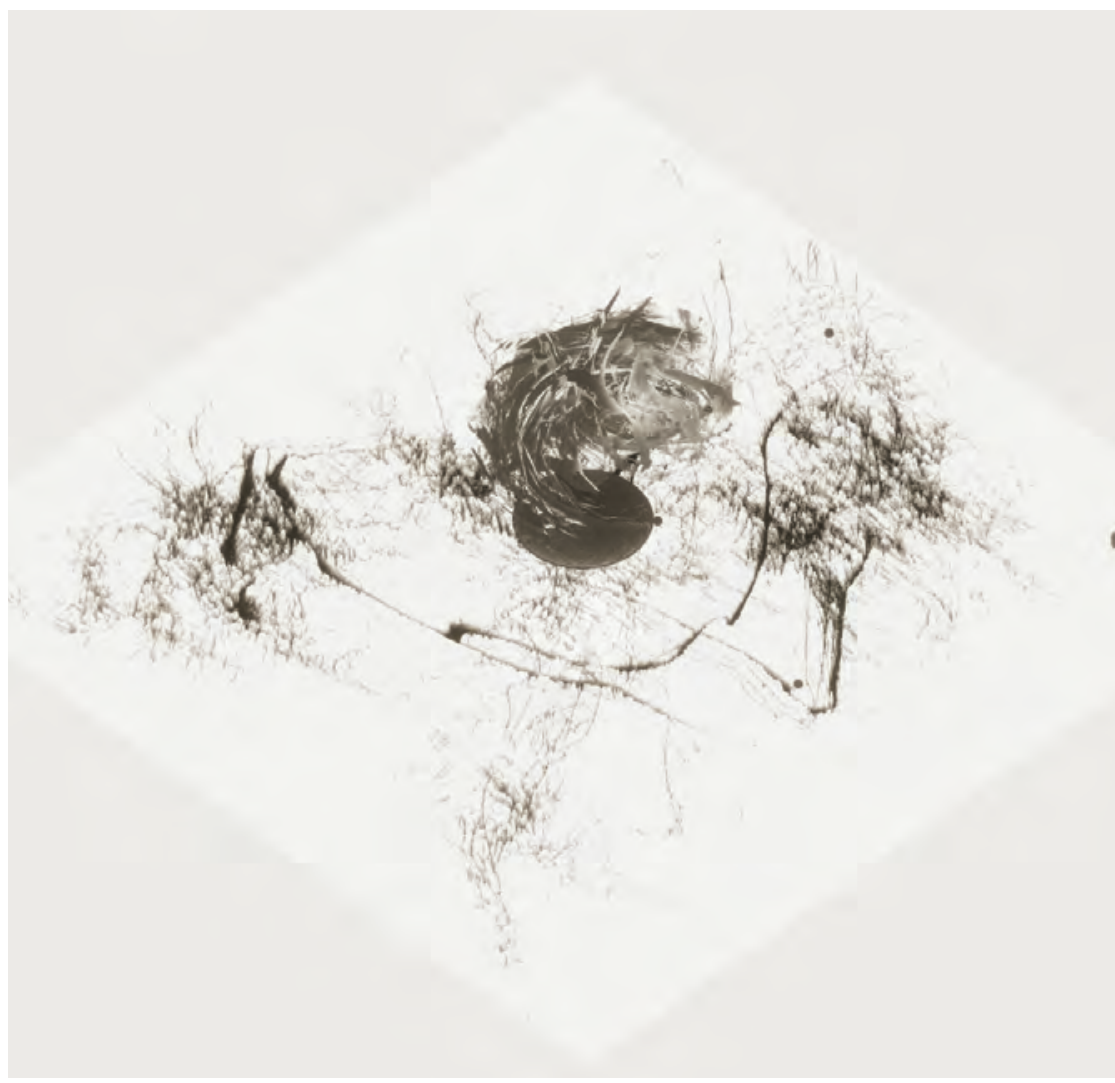

FIG. 50 2D DRAWING AS 3D SPACE - A 2-DIMENSIONAL PLANE IS TEXTURED WITH AN INK SKETCH
EXPERIENCE

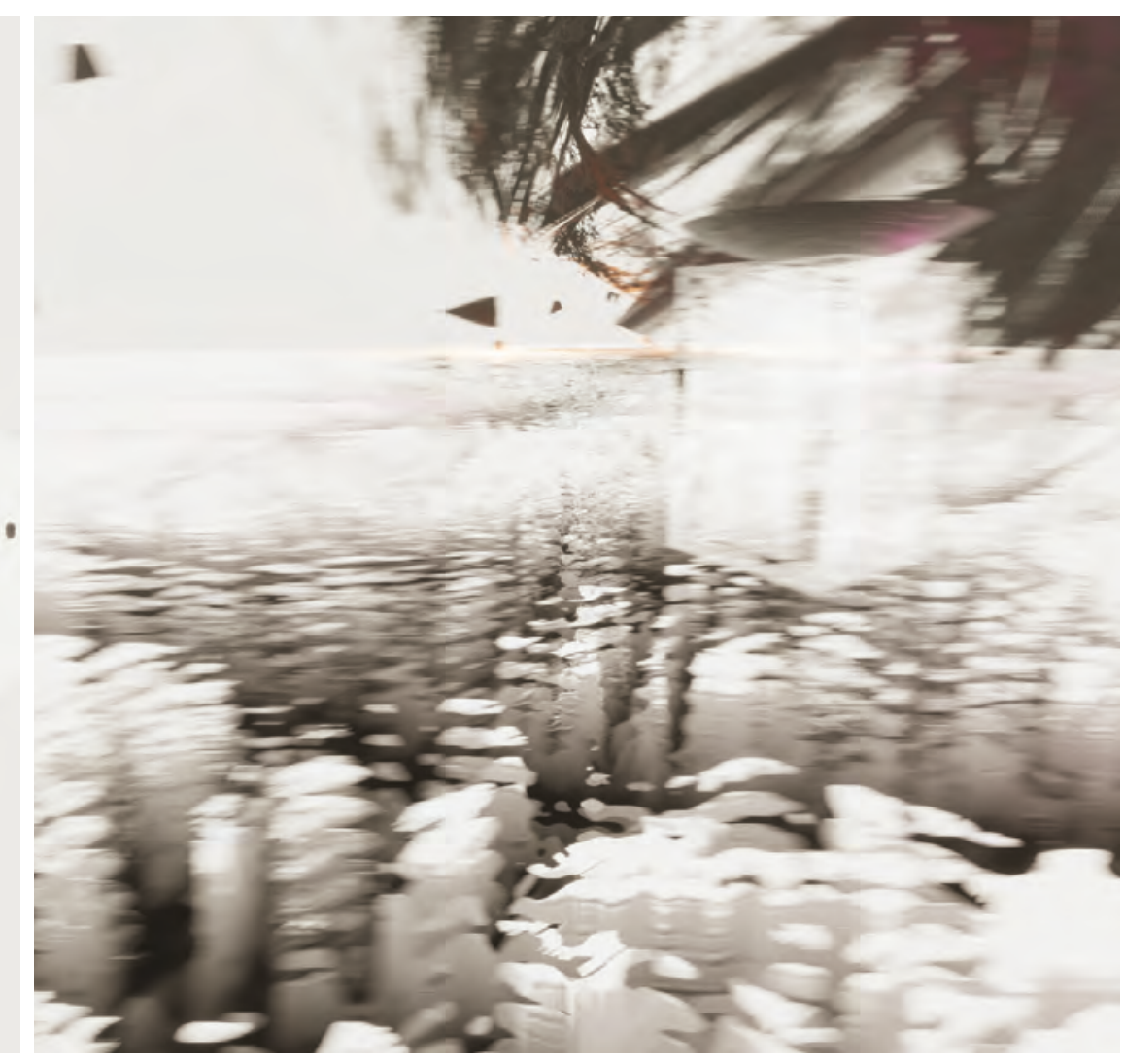

FIG. 51 2D DRAWING AS 3D SPACE - PIXEL DISPLACEMENT RENDERS BLACK STROKES AS DEPTH 
Level 1 Gameplay Videos

Level 1 Gameplay with Sound Effects

https://www.youtube.com/watch?v=G|4btmwS95Y

Level 1 Early Gameplay

https://www.youtube.com/watch?v=ZBgNwfwppmU\&t=5s

Level 1 Lighting Test

https://www.youtube.com/watch?v=h12Fmm5sJTc 


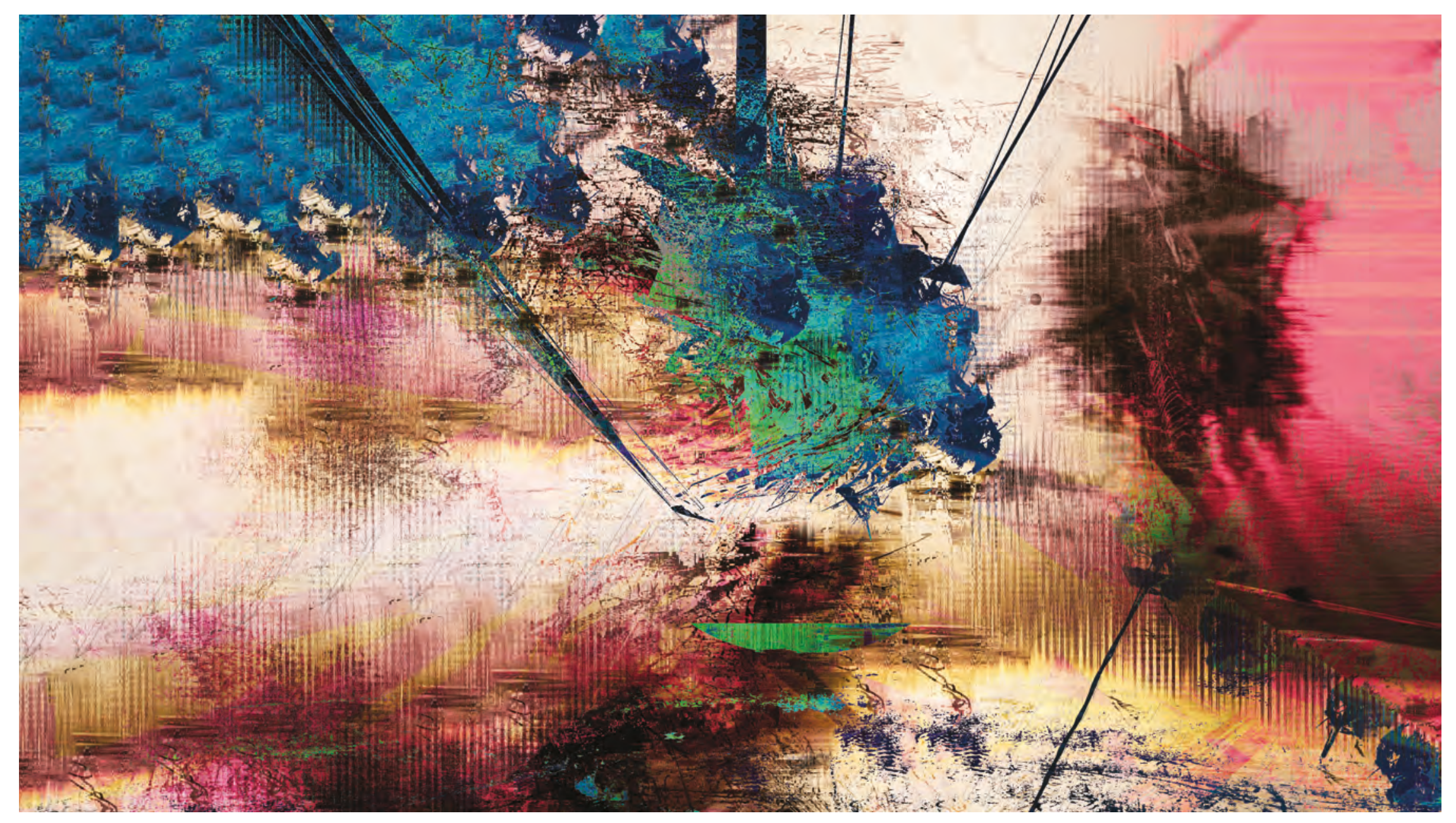

FIG. 52 LEVEL 1 GLITCH COLLAGE 
LEVEL

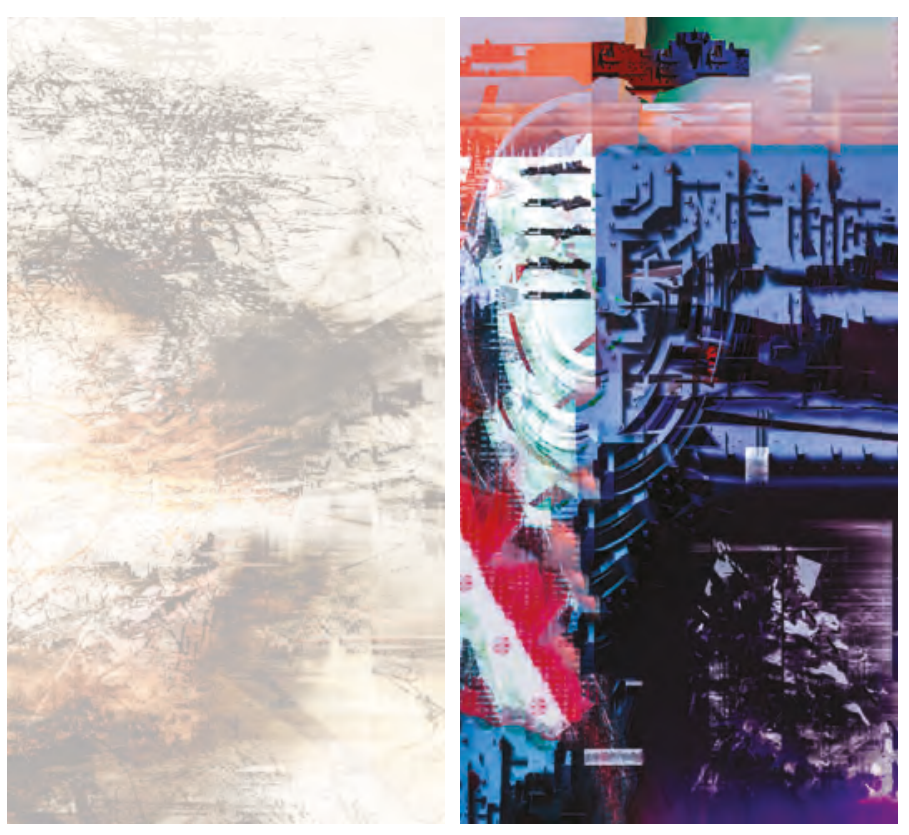

INTERACTING WITH THE

3-DIMENSIONAL
LEVEL 3

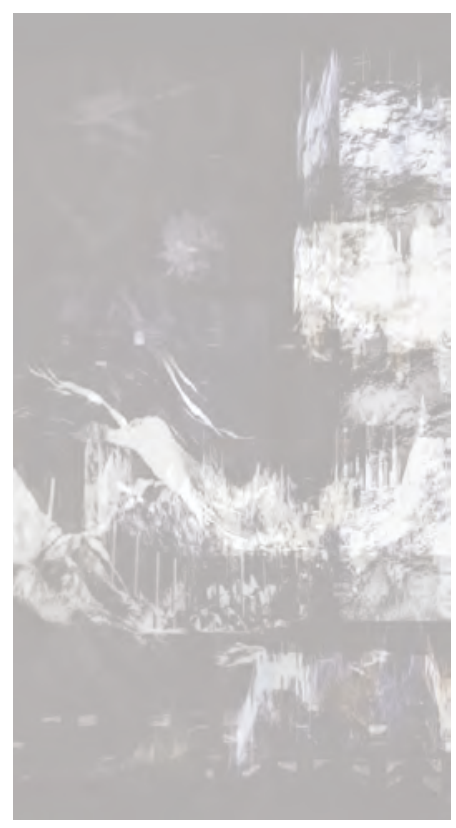




\section{LeVel 2}

For the second level, I re-use a classic level from Halo. Halo was an influential game during my childhood. I remember many family moments with my family (brother, parents, cousins...) playing that level. Halo and its levels are now considered classics in the videogame community. The level in my game is a recreation of one of the maps from Halo titled "Hang 'Em High". This map was in the original game and re-imagined in sequels of the Halo franchise. It was so popular that it was continually iterated by both the developer and the gaming community. The map was recreated with the intention that every completion of the map resulted in a new geometry. The form of the level was recreated in Rhino using a screenshot from the games loading screen. The height of the "tombstones" was estimated at two meters which informed the height of the buildings and bridges. Continual tweaking the scale of the level was necessary until the original game was accurately recreated in the Unity Engine.

A game mechanic was needed in order to transform the levels space. The sphere was used due to its ability to roll after throwing and dropping. This would cause the formal elements that the sphere is connected to, through the game mechanics to deform- even after the player lost control of the sphere. In the first level, intractability was resigned only to the objects within the space, not the space itself. In the second level, the reverse is true. Connecting the geometry of the level to the interactable sphere laves the player disoriented. Navigational difficulties provide a challenge for the player to overcome. The act of trying to progress through a level that the player character was actively obfuscating is difficult- and fun. In order to provide an end goal, I used the cauldron from the first level, this time at a much smaller scale. The player must use their character to bring the sphere to the cauldron in order to complete the level.

Another opportunity presented itself to implement areas of the level where the players view is no longer connected directly to the player. These areas provide a greater challenge as the player moves through the level and even change locations upon subsequent completions.

I begin looking at a peaceful scene on top a monochromatic platform. Bridges span between two opposing structures. At the highest point of the structure across from me I notice a small crucible, it has changed places since the last time I played. The sphere beside me is glowing with an ominous red. When I pick it up my vision becomes distorted and the structures around me contort in impossible directions. Bridges erupt and columns bend, the tombstones shift on their spots. As I attempt to reach the goal atop the far structure, I drop the ball and it is consumed by the forms around it. I must proceed through the glitched representations of the architecture to regain the ball. The edges and planes are warping and moving along with the ball as it rolls further from me. The planes appear solid, but they do not have any effect on my character, and I am able to pass through them to grab the ball. When I manage to reach my goal and place the ball in the cauldron, I am left standing in the same place. This time the gnarled architecture remains. I begin again continuing the process of the platform's endless growth. 
REPRESENTATION

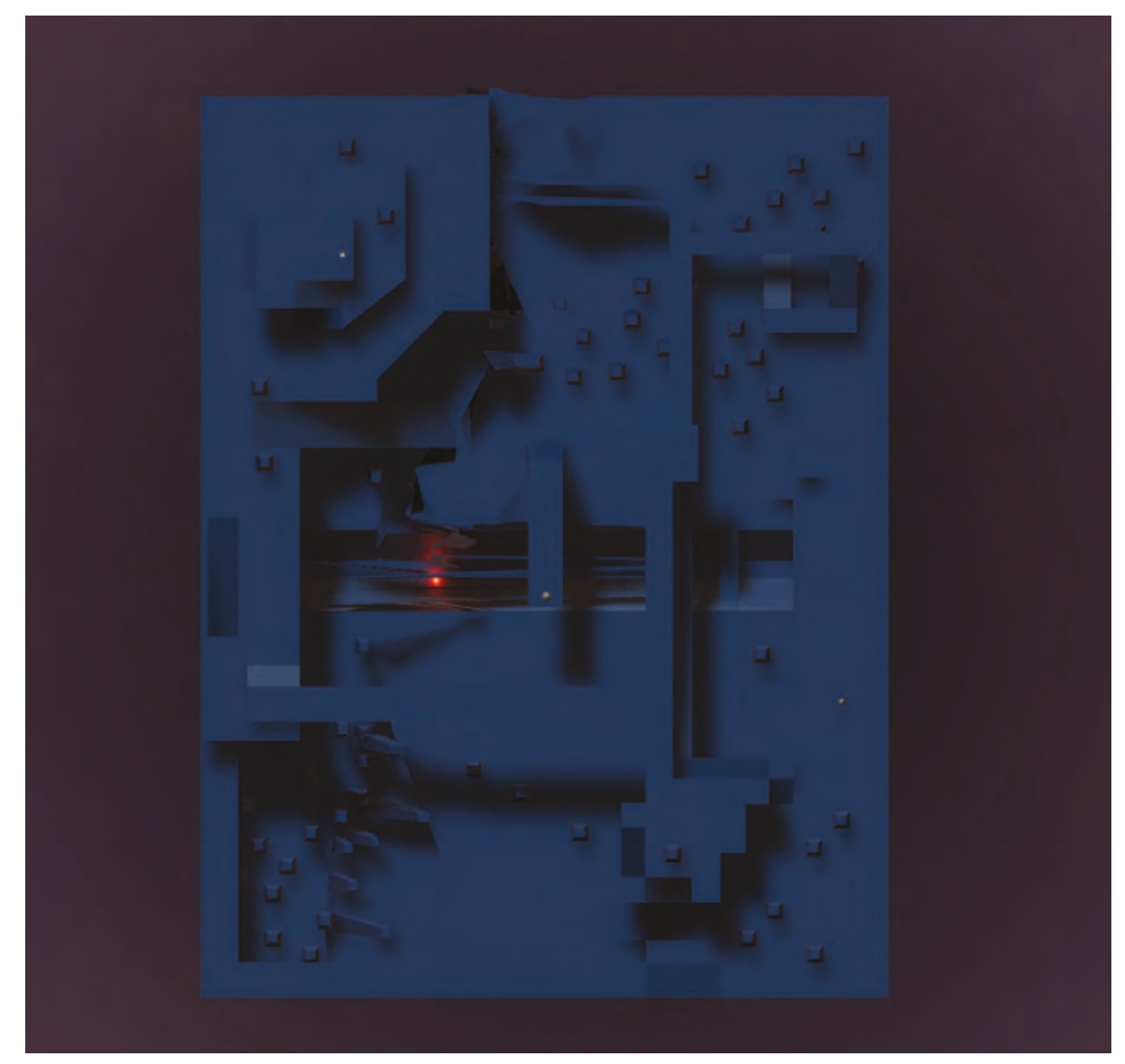

FIG.53 PLAN - A RECREATION OF HANG EM HIGH, A MAP FROM HALO CE
EXPERIENCE

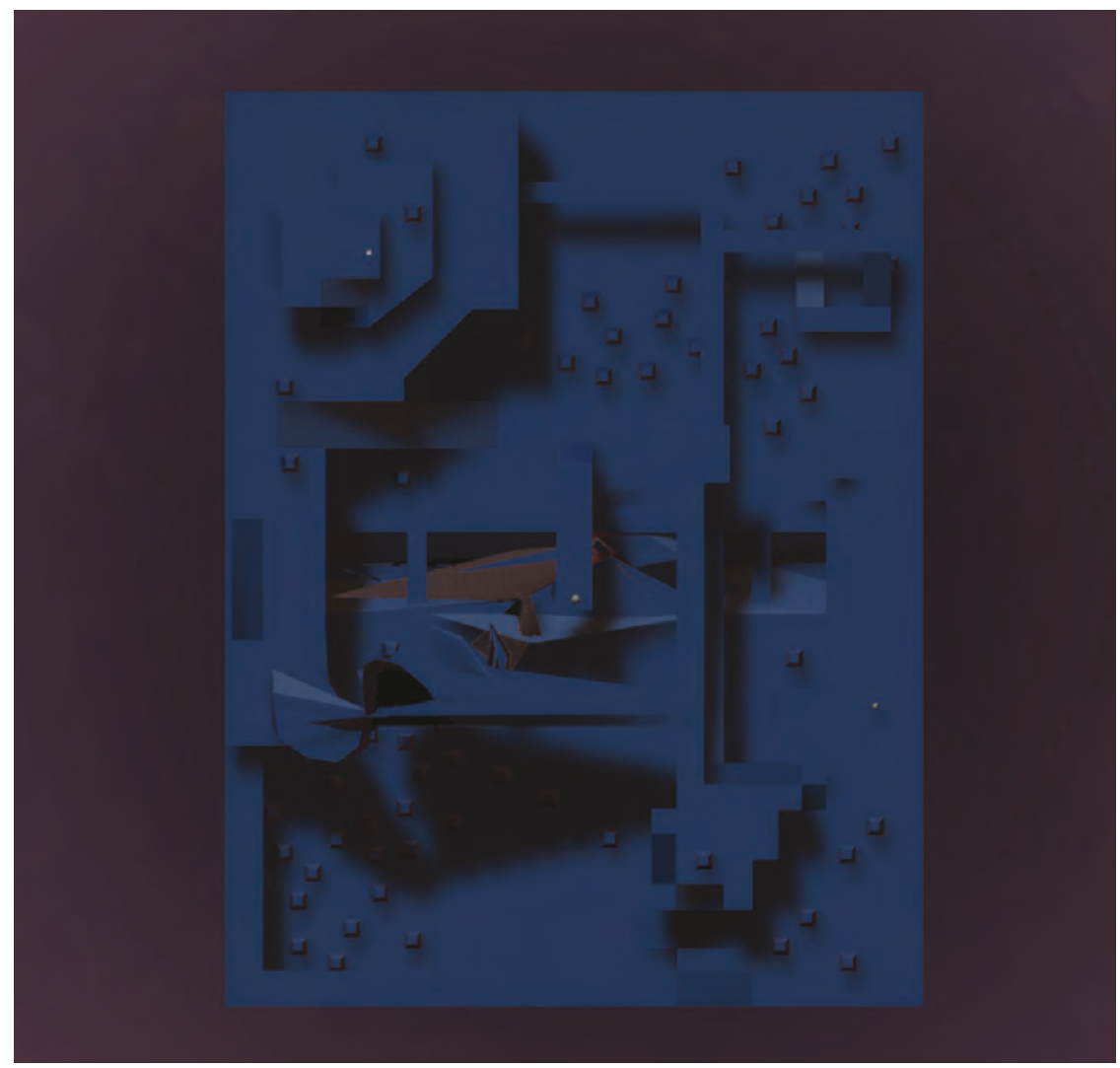

FIG. 54 PLAN - THE PLAYERS GOAL IS TO BRING THE SPHERE TO THE NOW MUCH SMALLER CAULDRON ON THE OPPOSITE SIDE OF THE MAP 
REPRESENTATION

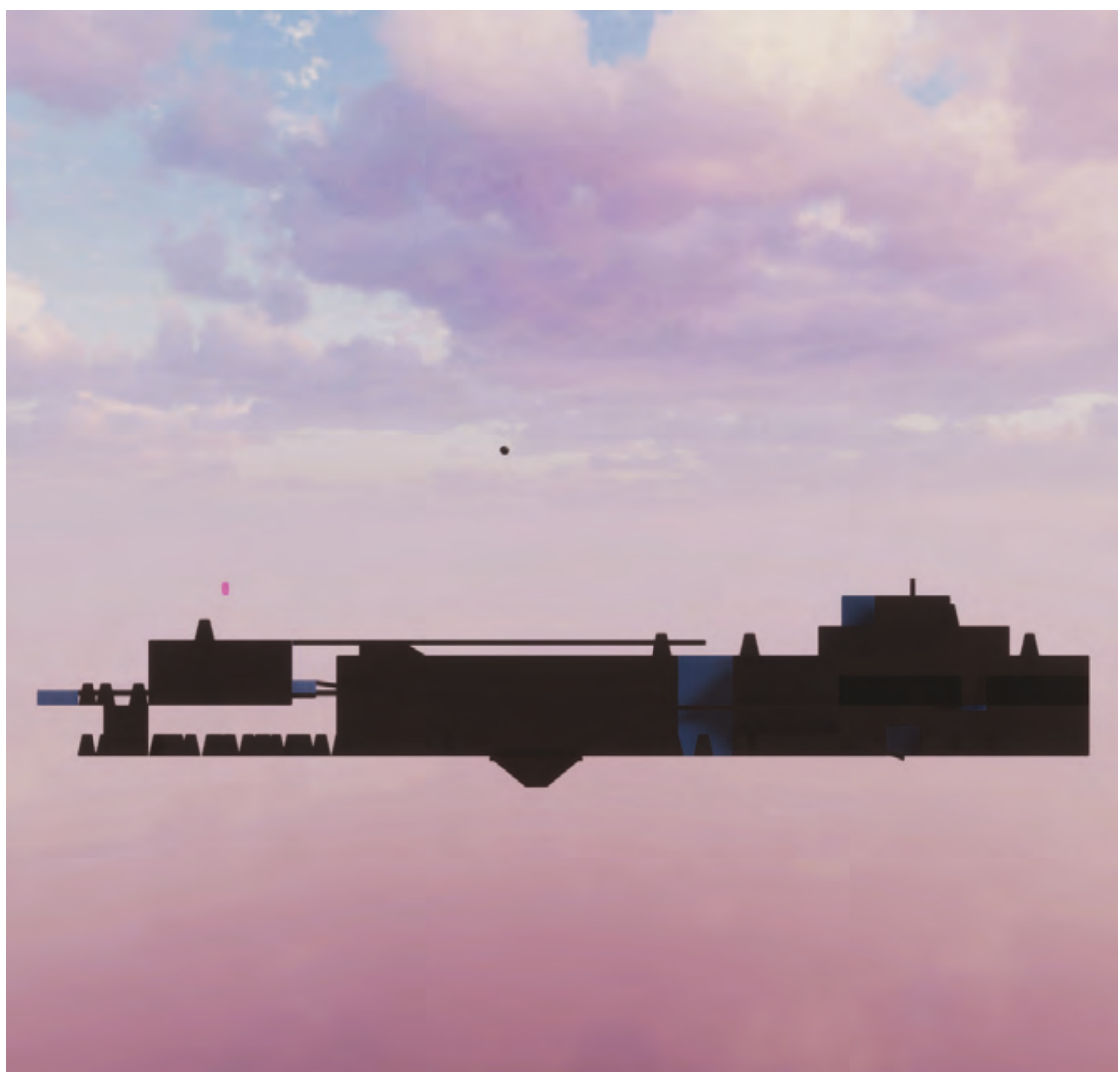

FIG. 55 ELEVATION - Undisturbed origINAL MAP BEFore the GAME HAS BEGUN
EXPERIENCE

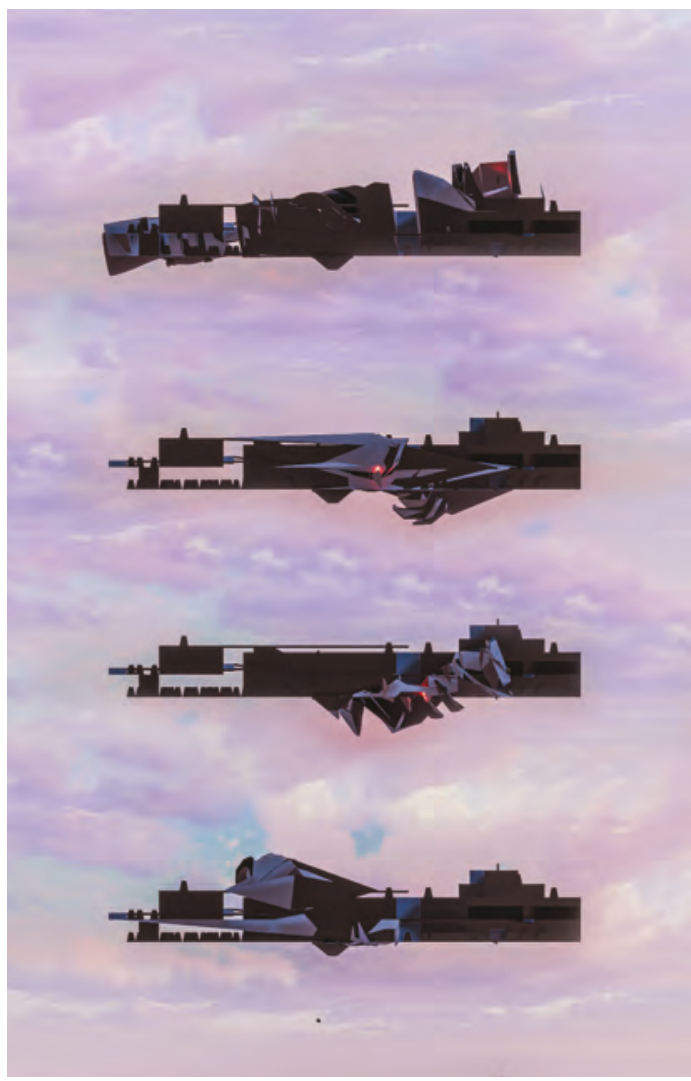

FIG. 56 ELEVATIONS - AS BALL MOVES TOWARDS THE OBJECTIVE 3D GEOMETRIES BEGIN TO DEFORM AND GLITCH, HINDERING THE PLAYER AND CHANGING THE LAYOUT OF THE LEVEL. THESE GEOMETRIES ARE THE RESULT OF PLAY WITHIN THE GAMES CONFIGURATION. 
EXPERIENCE
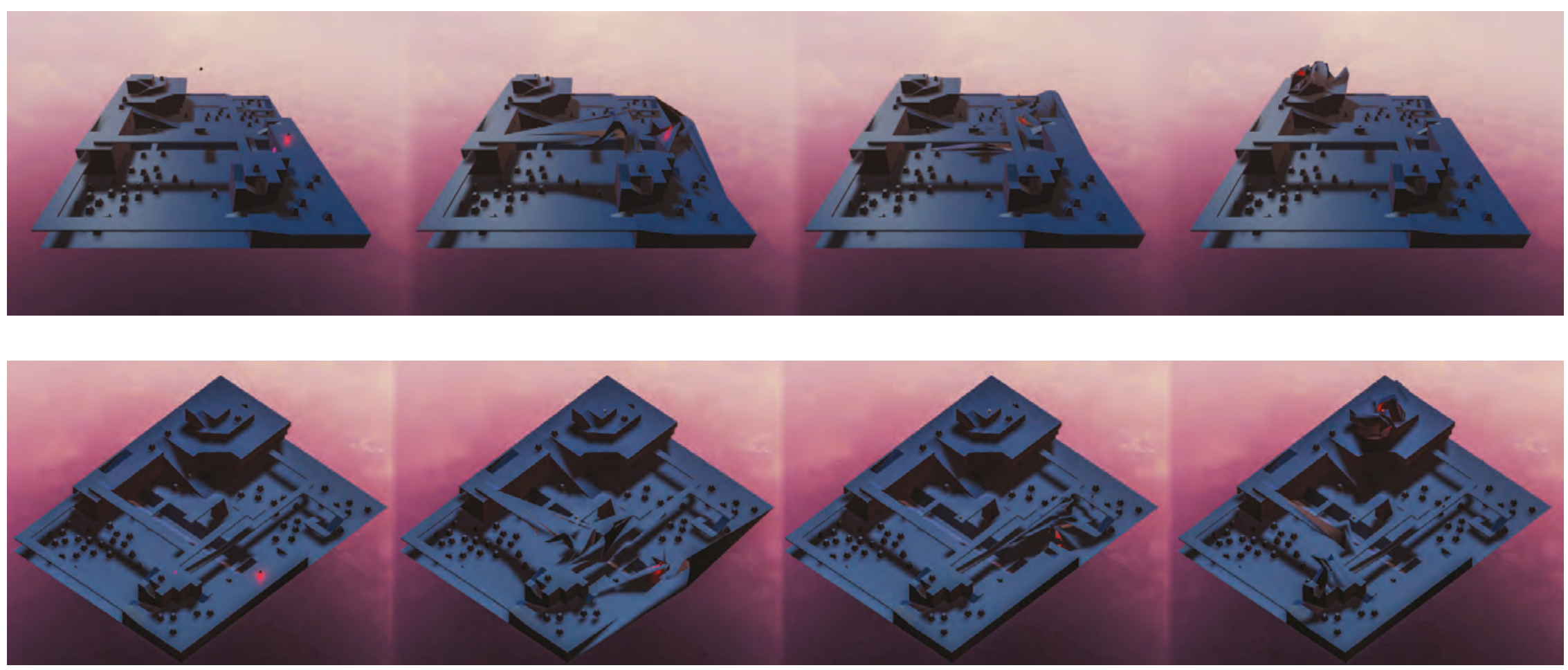

Fig. 57 Axonometric And PeRspective Views during Gameplay - The geometries take NEW SHAPES AND "BREAK" THEIR STANDARD MESH MATERIAL RENDERINGS. 
REPRESENTATION

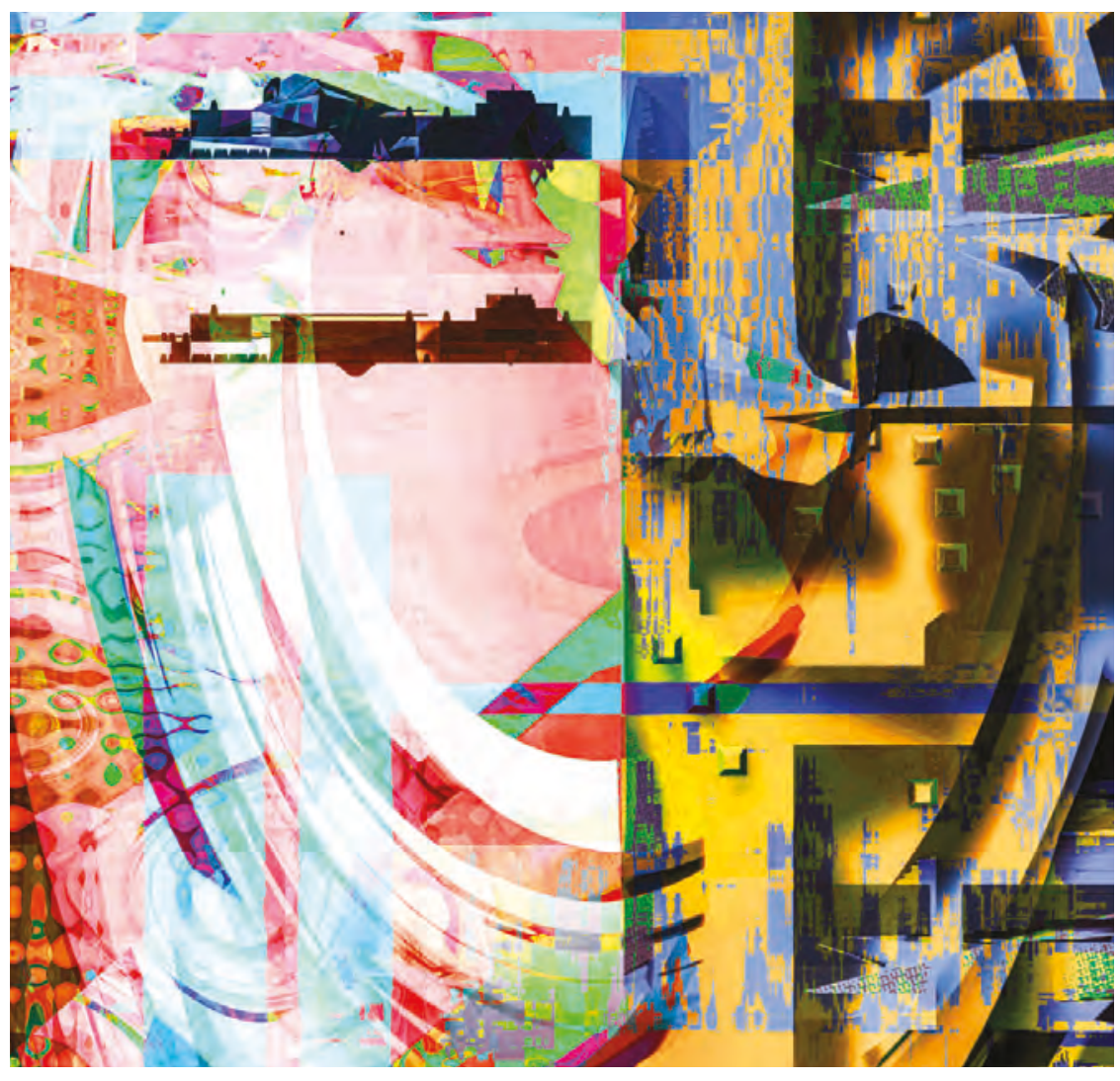

Fig. 58 New LeVel Painting - Upon completion, the LeVel could Generate A new MESH BASED ON THE MOVEMENTS FROM THE PLAYER. A NEW LAYOUT WOULD BE GENERATED BASED ON THE REPRESENTATION OF 3D SPACE THAT WAS LEFT BEHIND BY THE PLAYER RESULTING IN A NEW EXPERIENCE WITH DETAIL ABOVE

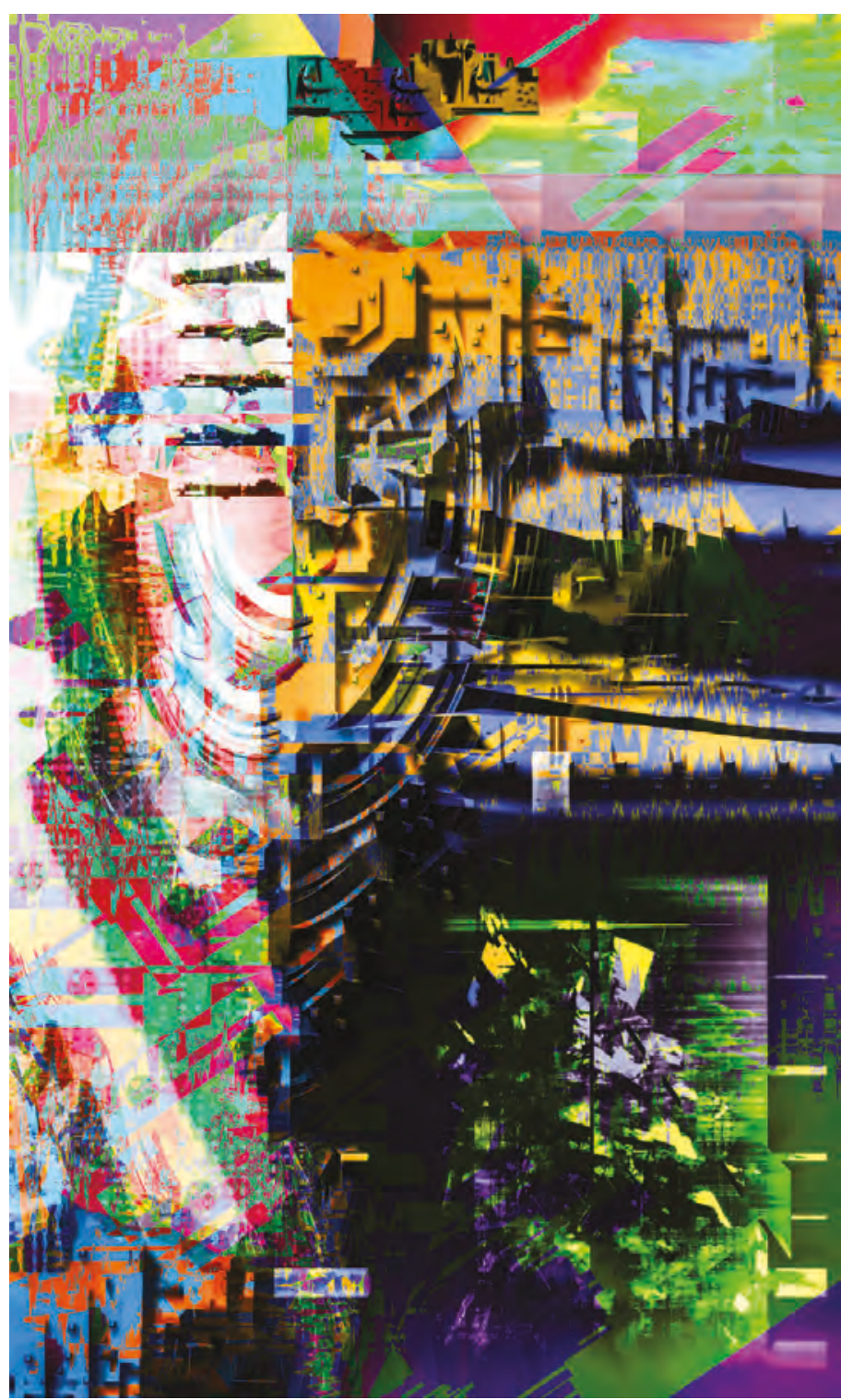


RepresentATION

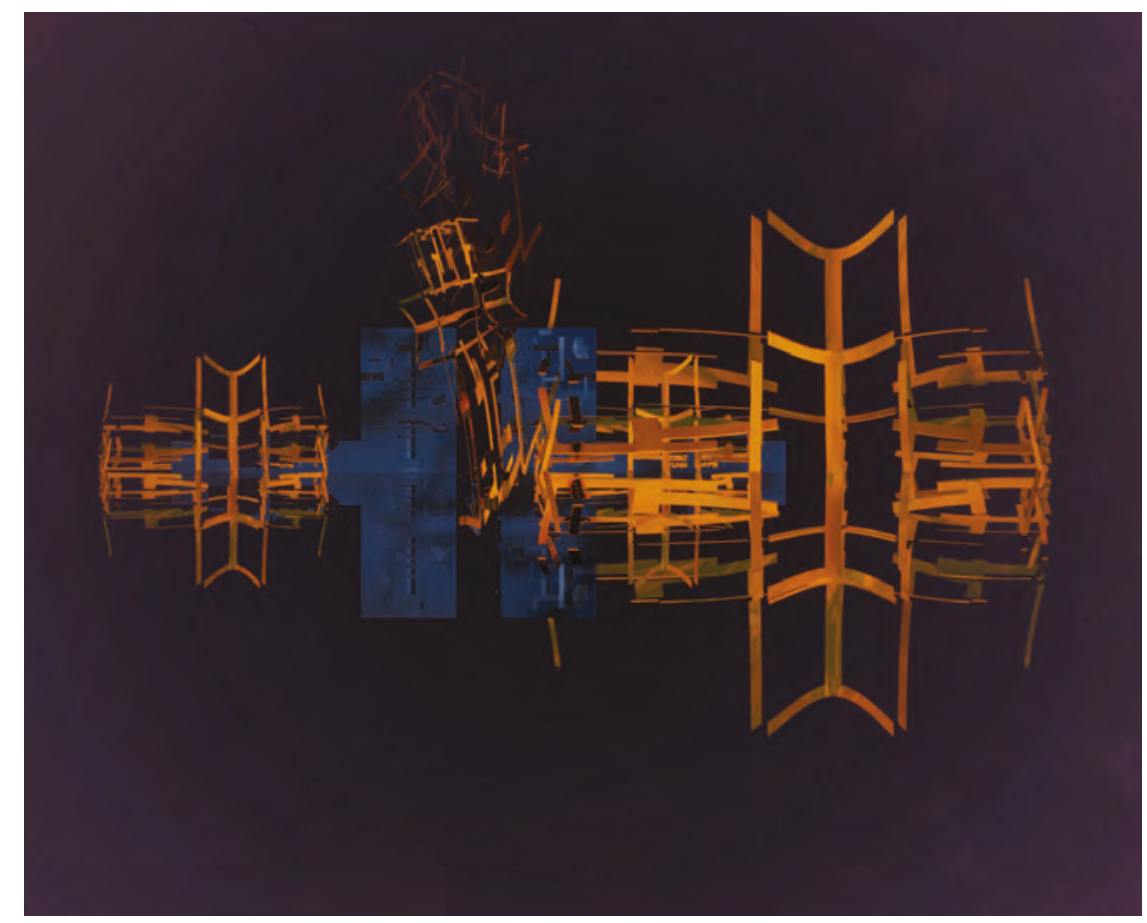

EXPERIENCE

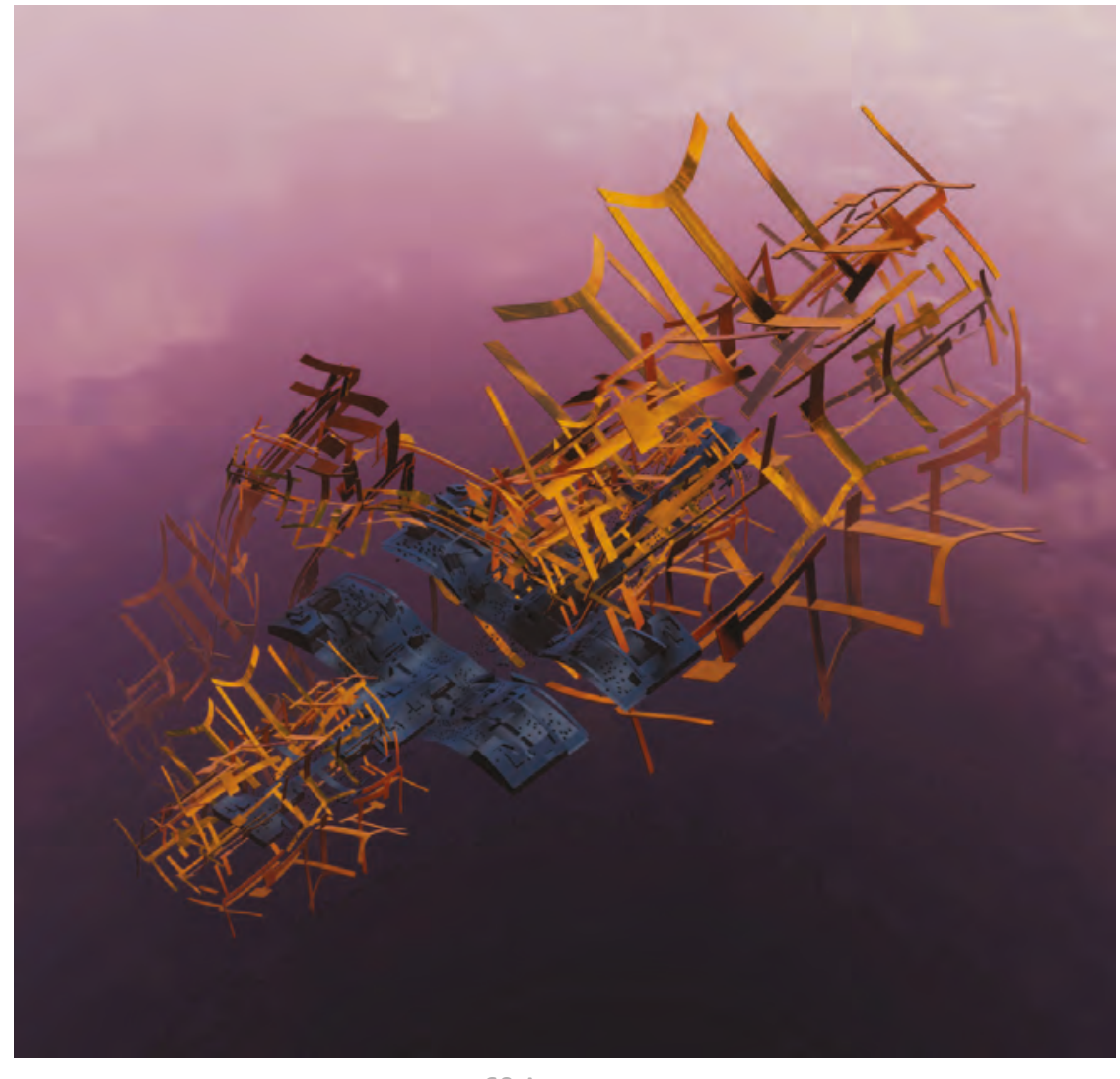

FIG 60 AXONOMETRIC 
EXPERIENCE

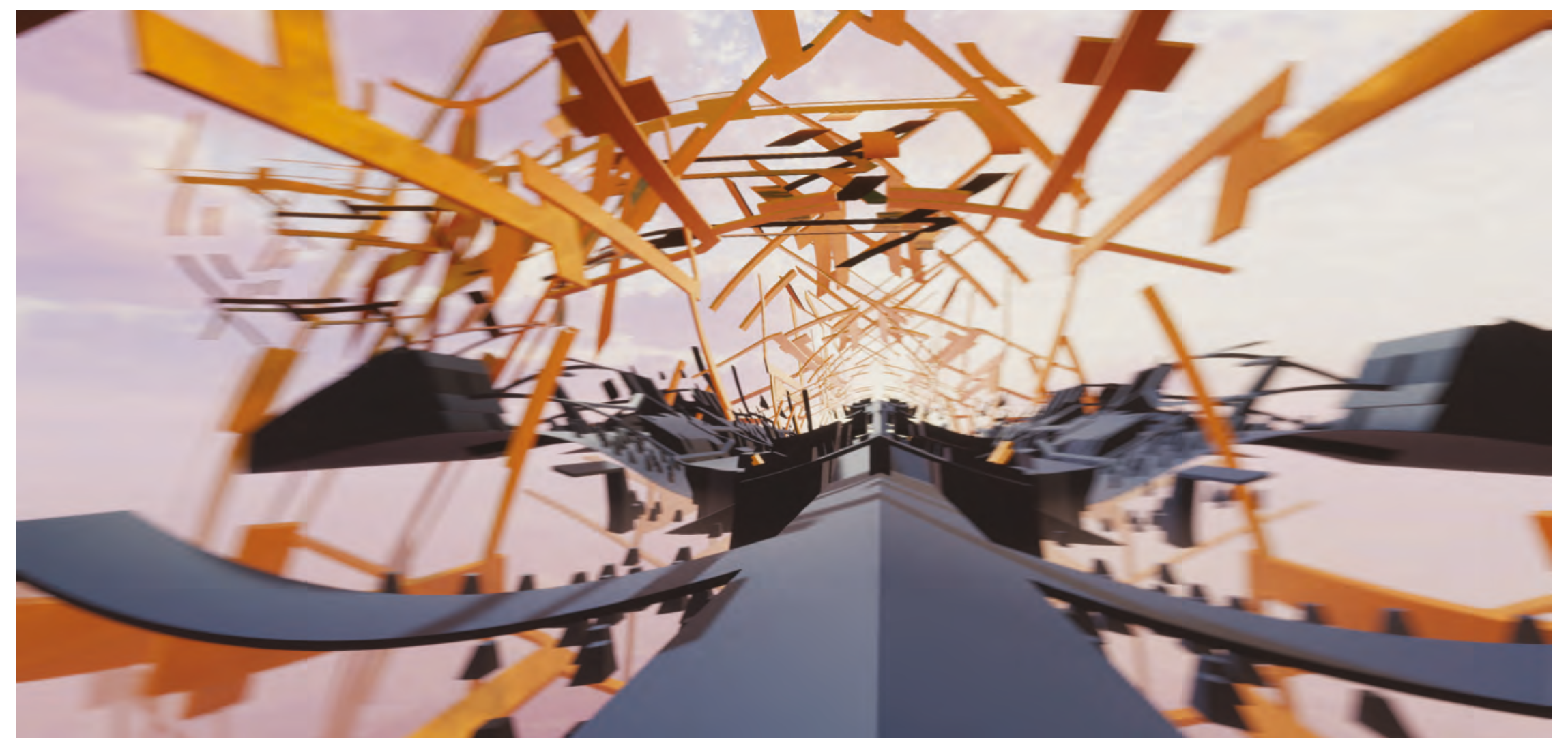

fig. 61 Perspective - Playing the new map 
Level 2 Gameplay Videos

Level 2 Gameplay with Sound Effects

https://www.youtube.com/watch?v=Lb9wcOSTXLo

Level 2 Early Gameplay

https://www.youtube.com/watch?v=j6ON_MSvlcU 


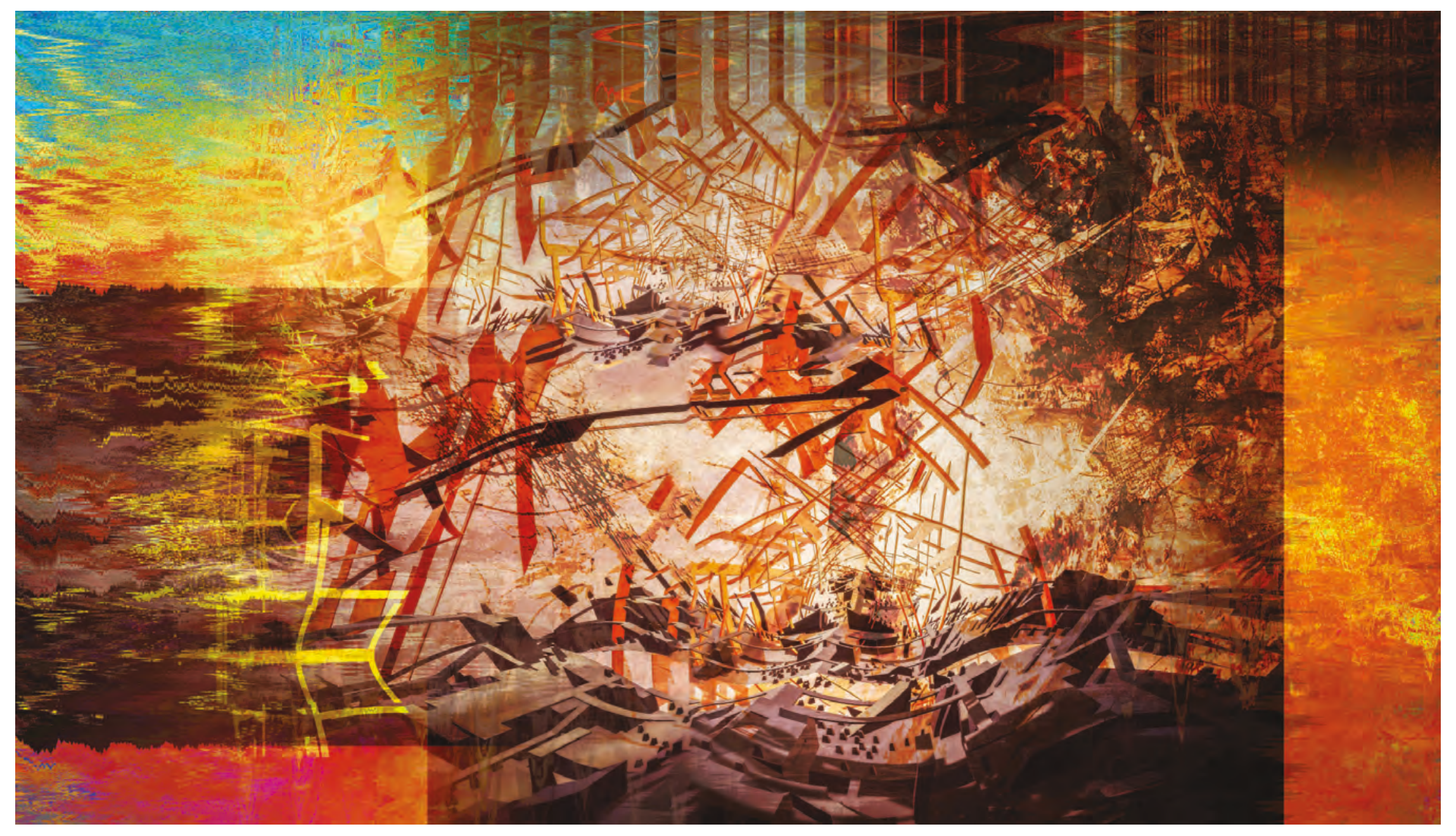

FIG. 62 DIGITAL PAINTING OF CONTINUED GENERATION 

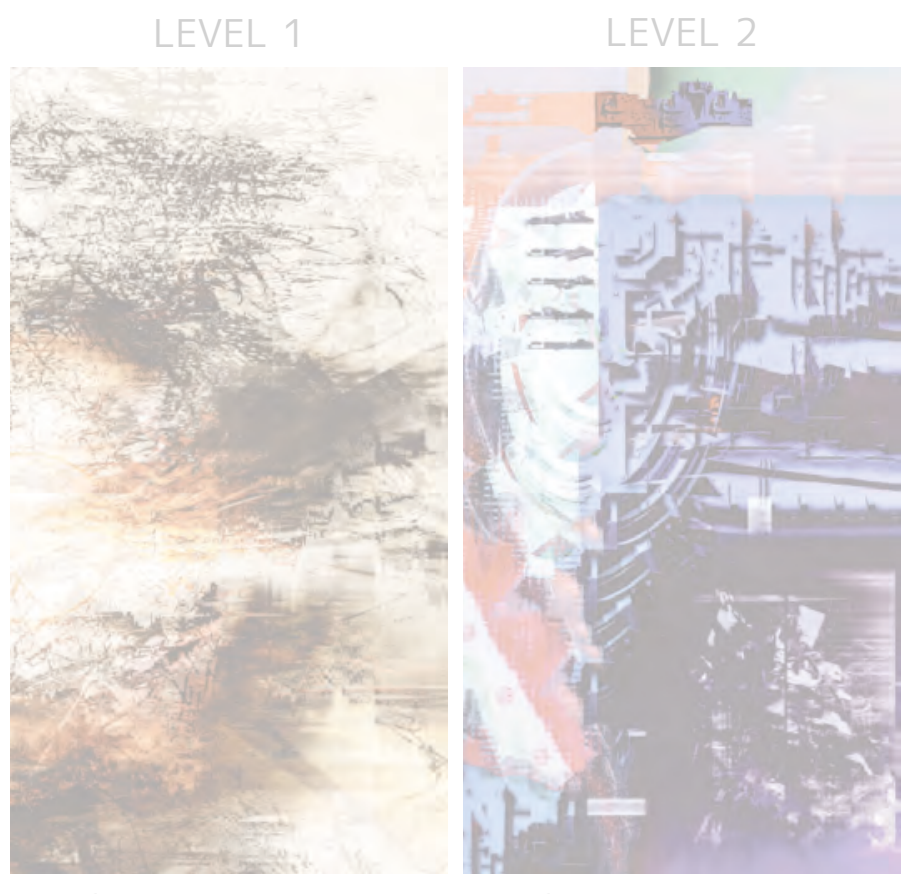

LEVEL 3

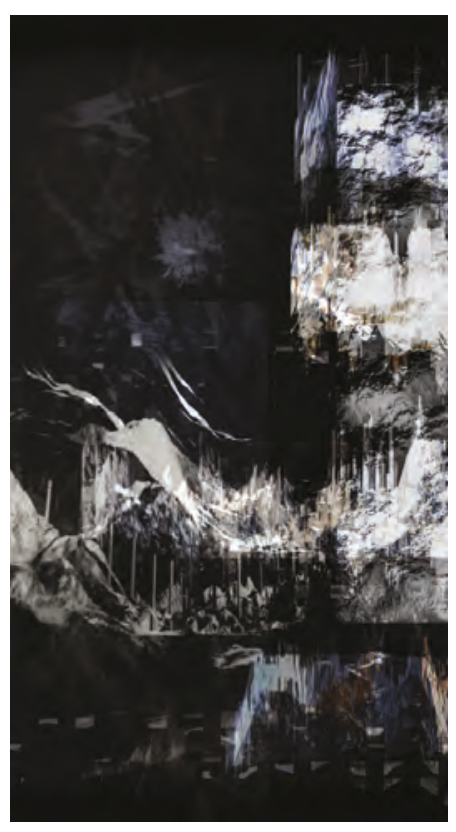

EXPERIENCING VIRTUAL SPATIAL

POSSIBILITIES 


\section{LeVel 3}

The final level combines the mechanical lessons learned from the previous two levels with a representation of the actual world. This level allows for the manipulation of both objects and environment. One area where digital technologies excel is high-definition natural environments. This level takes inspiration from video game concept art explored through graphite sketches. A snowy mountain environment was sculpted using the Unity terrain editing tools. Focus was placed on designing an immersive environment that could be explored for a longer period. To develop the terrain, Unity has terrain tools that use a set of brushes to stamp a 2-dimensional plane. These brushes can raise, lower, stamp, sculpt, erode, flatten, the surface to form topography and to populate that topography with natural features like trees. The level was designed as a journey upwards with two branching paths to explore, each path leading to the same endpoint. A hill splits the path directing the player to either side. In the first level, an invisible wall prevented players from leaving the game space. In this level raised mountains are used. The natural environment blocks the players path which reinforces greater immersion while exploring the level. Both paths lead up a steep hill that tests the players ability to traverse vertical obstacles. At the top, the player is funneled towards a view of the skybox where a sphere has already been placed on its cauldron. The journey to the sphere is designed to immerse the player in a realistic virtual environment. Returning with the sphere reveals the nature of the sculpted terrain. The player can see that the giant mountains are merely 2-dimensional hills that are floating in space. The journey back is meant to reveal the fabric of the game space, beginning with there immediate environment. If the player chooses to bring back the sphere to where they began the contents of the level begin to shift in relation to the location of the sphere.

I am unsure where I am, surrounded by snow and mountains. Two branching paths lead towards the mountain tops. Along the path I encounter surreal scenes. Architectural elements merge with environment, some are even moving. The experience is dreamlike, realistic but not everything I am seeing follows logic. After exploring a path, I encounter the same 2-dimensional drawing from the first level. It hovers over the mountains welcoming me towards the cauldron at its base. A sphere already sits inside of the bowl and I am tempted to remove it and return to where I began. As I return the weather worsens and the sphere begins to move and distort the environment. Trees arch away and rush towards me, rocks melt and crumble, the surreal scenes shake and shutter. The nightmare ends when I return to the place I began. 
REPRESENTATION

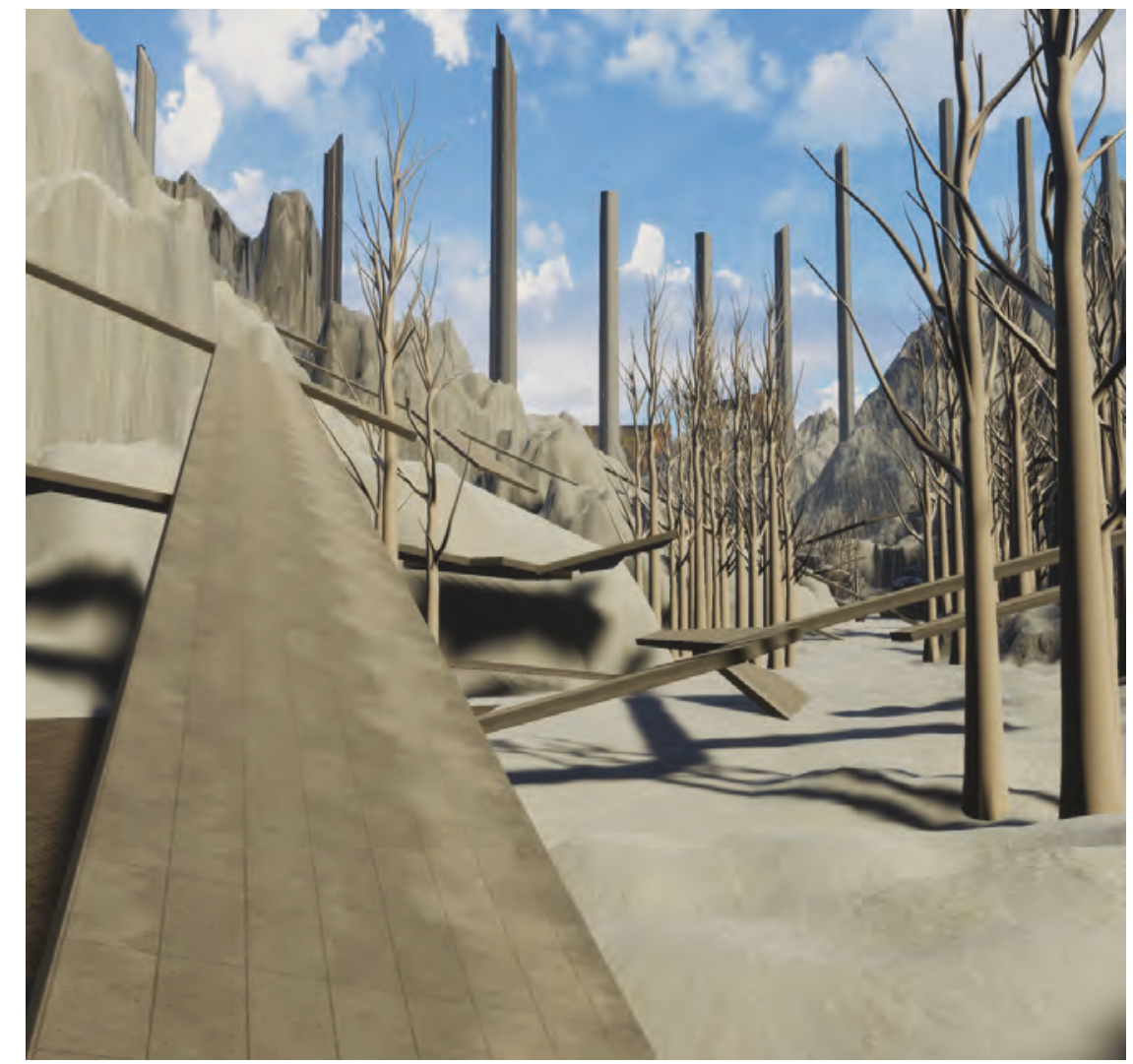

Fig. 63 PeRspective - THE LeVel offers a LARge Mountainous AREA to be EXPLoRED WITH DIFFRERENT SPATIAL AND MATERIAL EXPERIENCES
EXPERIENCE

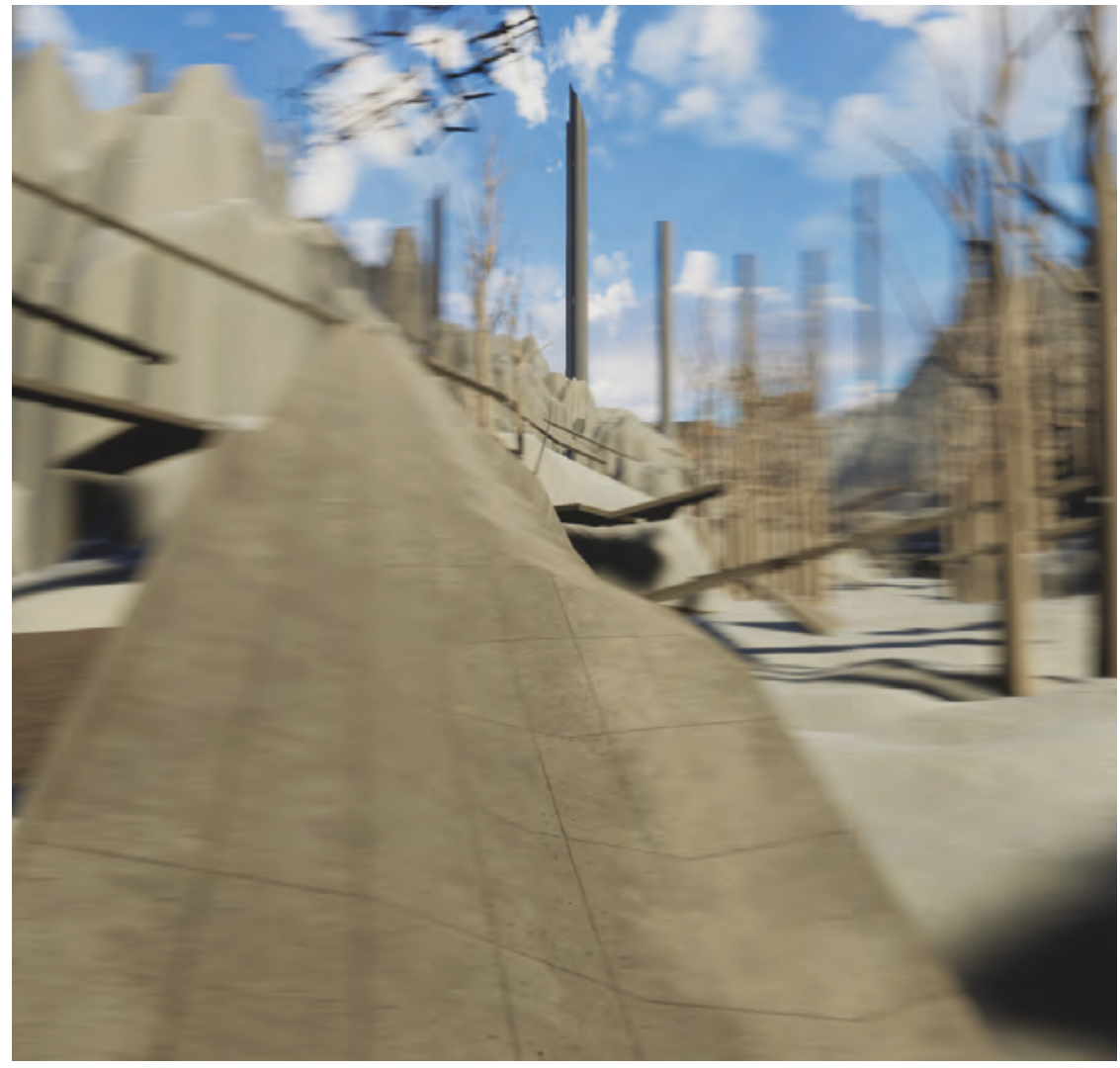

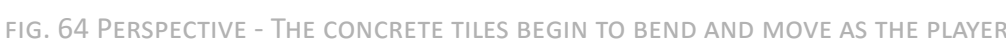
APPROACHES THE BRIDGES FROM LEVEL 2 NOW EMBEDDED IN THE SNOW 
REPRESENTATION

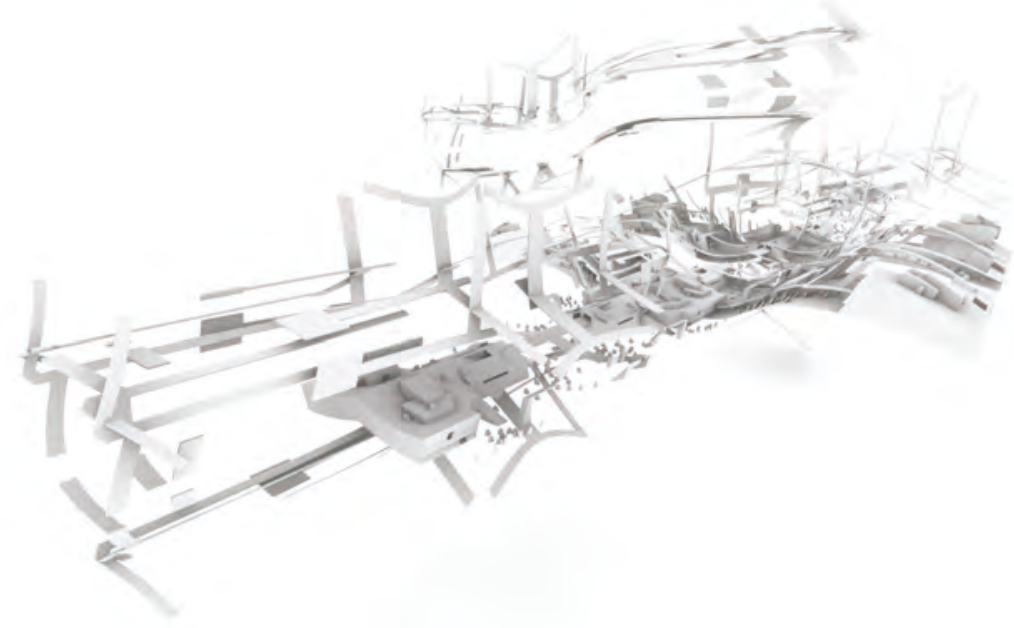

FIG. 65 PERSPECTIVE - LEVEL 2
EXPERIENCE

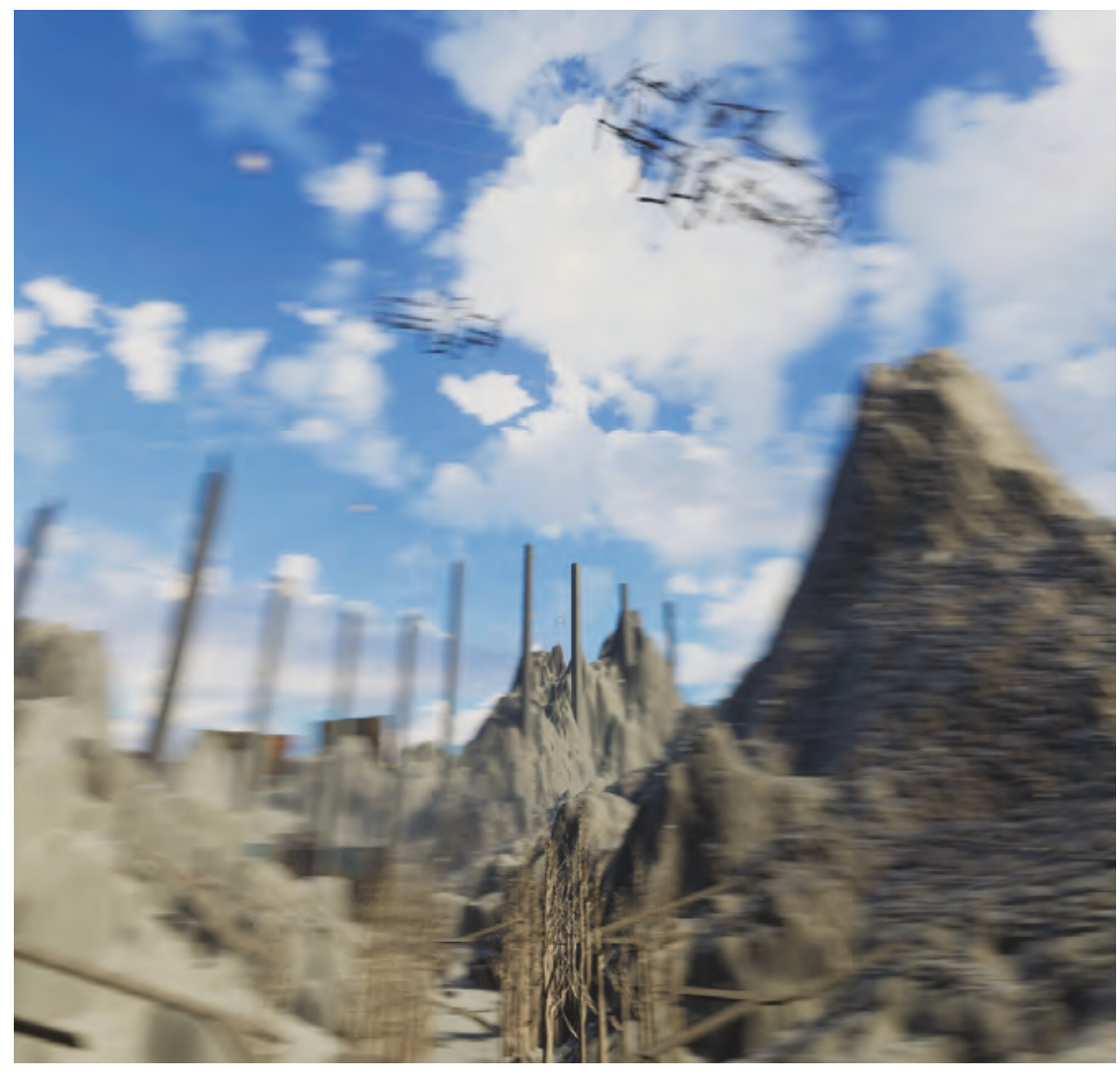

FIG. 66 PERSPECTIVE - LEVEL 2 ACTING AS A BOID, AT A NEW SCALE IN LEVEL 3 
REPRESENTATION

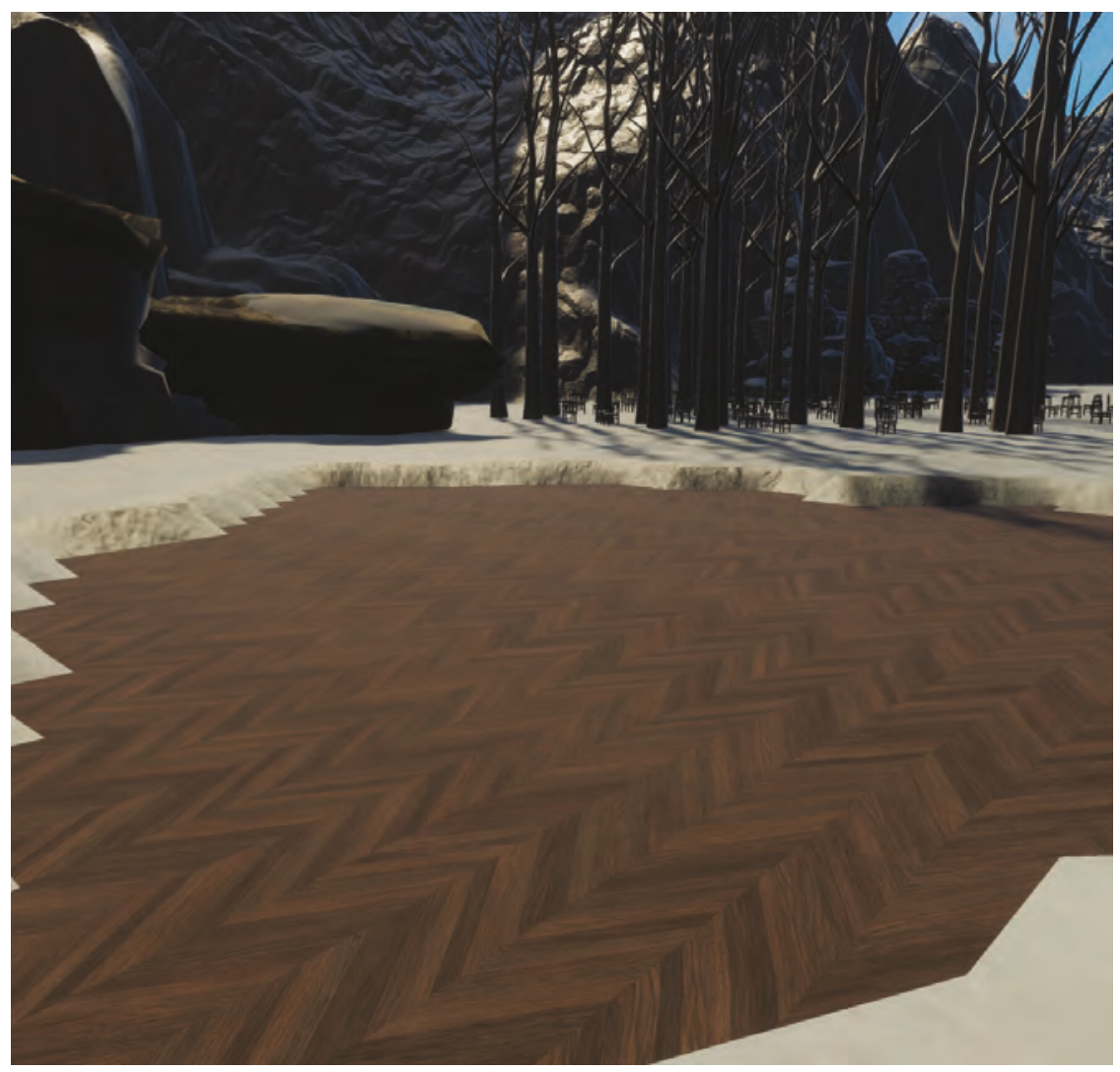

FIG. 67 PERSPECTIVE - A POOL OF WATER HAS BEEN REPLACED WITH WOOD
EXPERIENCE

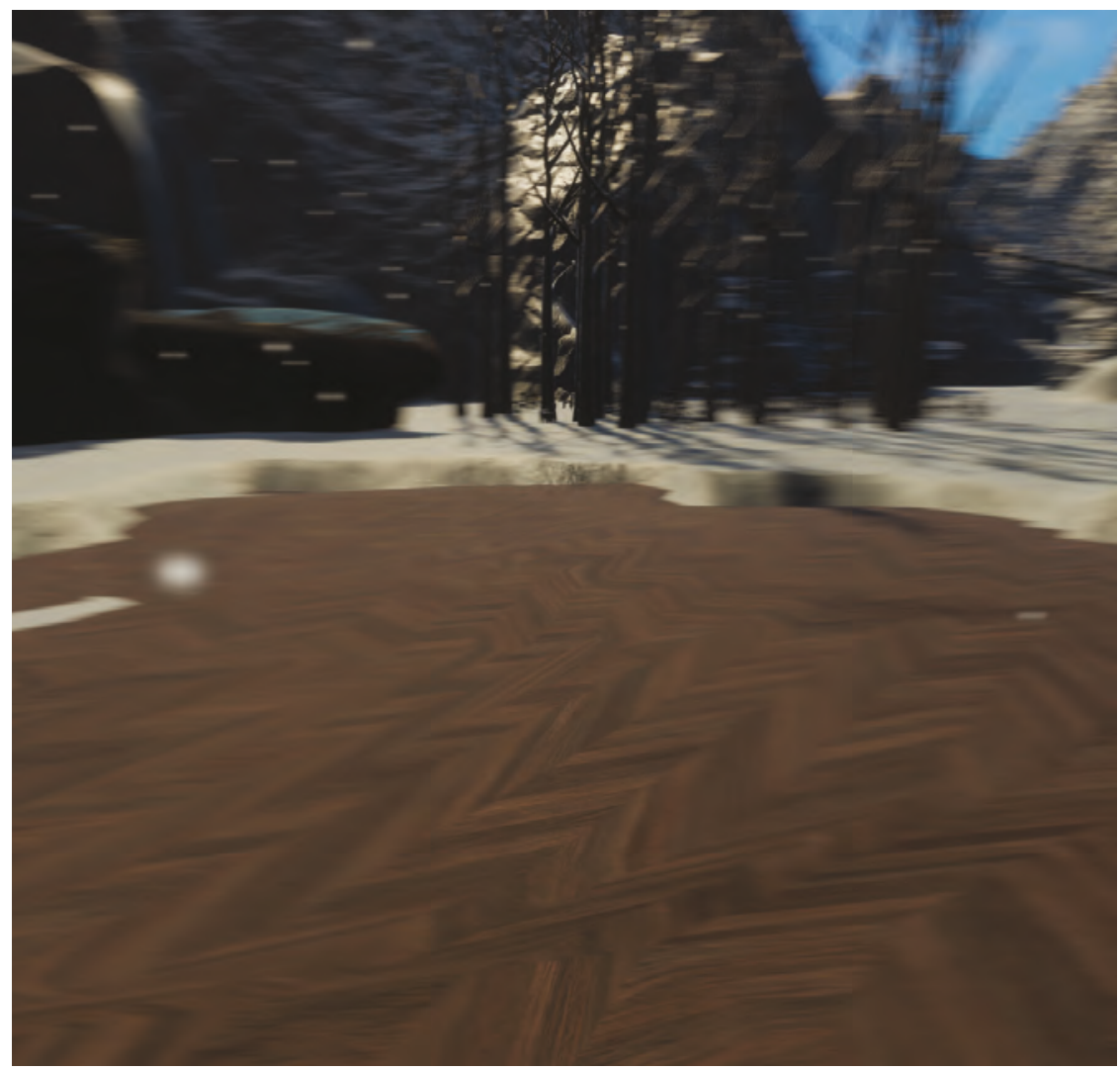

FIG. 68 PeRSPECTIVE - AS THE PLAYER WALKS PAST THEY CAUSES THE POOL OF WOOD TO RIPPLE AND WAVE 
REPRESENTATION

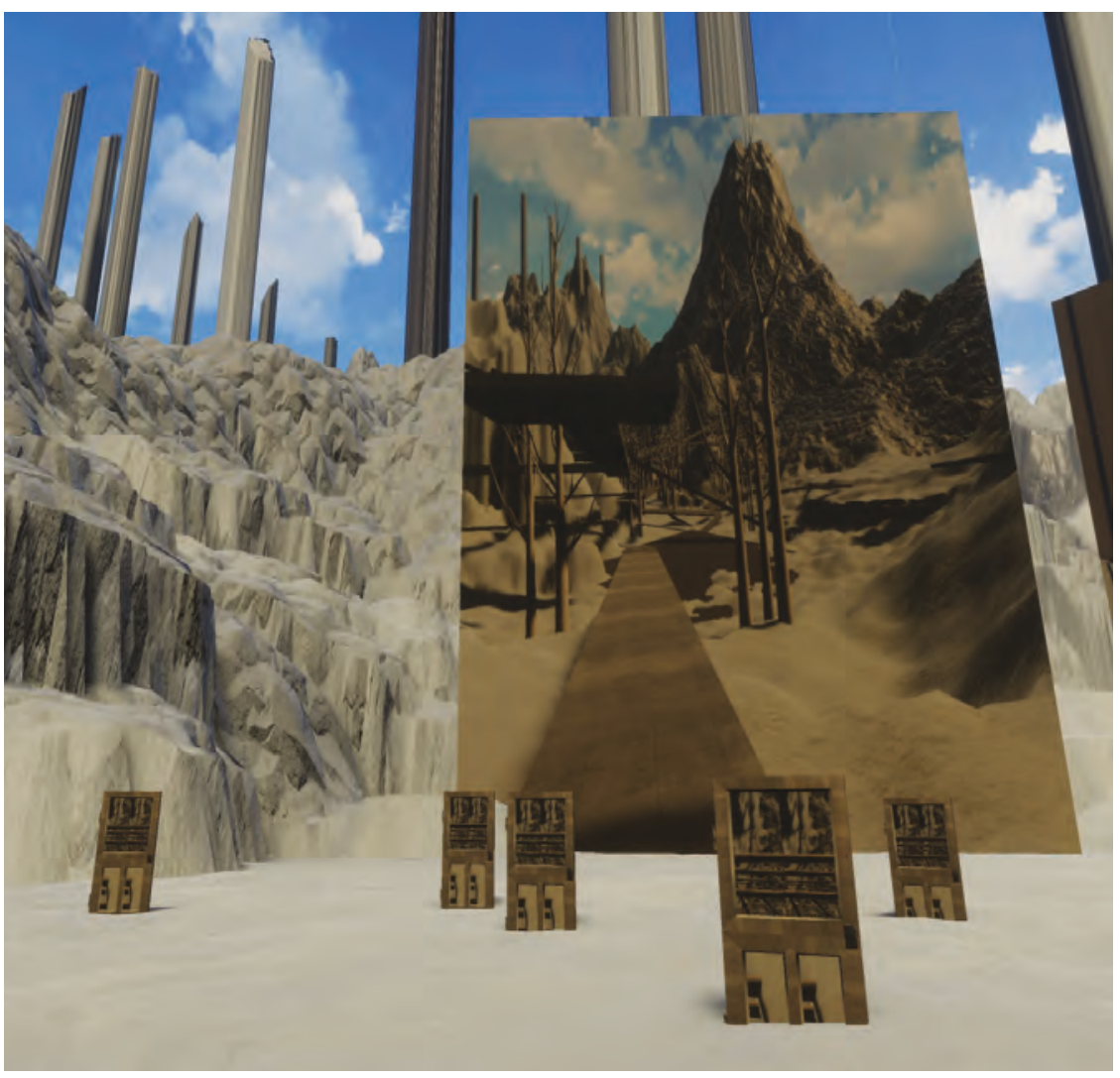

FIG. 69 PERSPECTIVE - LARGE PLANES, ROCKS, AND DOORS HAVE BEEN TEXTURED WITH A LIVE VIEW OF THE PLAYER'S PERSPECTIVE
EXPERIENCE

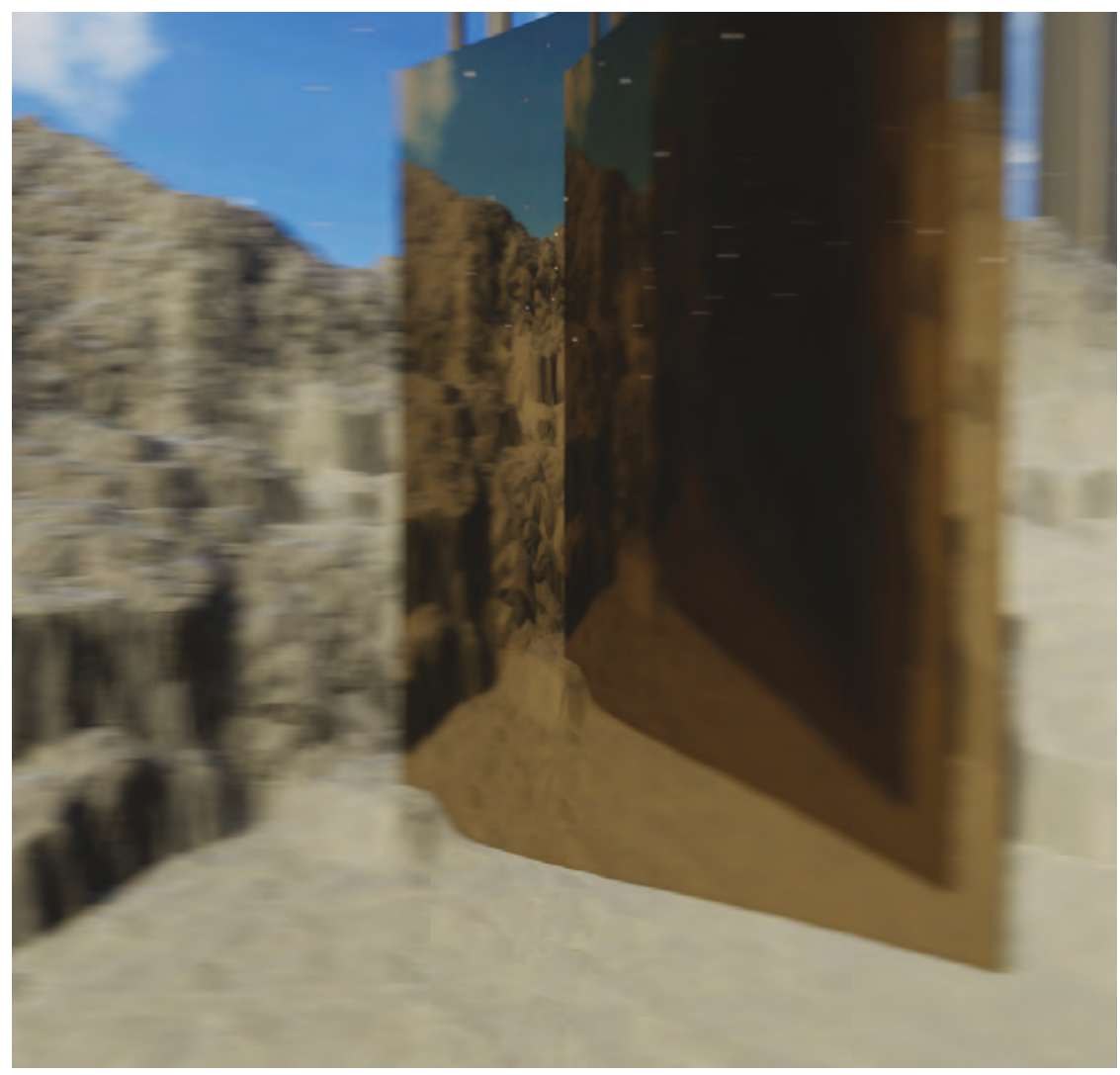

FIG. 70 PERSPECTIVE - AS THE PLAYER APPROACHES THE PANELS DISTORT, EXTEND TO INFINITY, OR SHOW THEIR PLANS AND ELEVATIONS 
REPRESENTATION

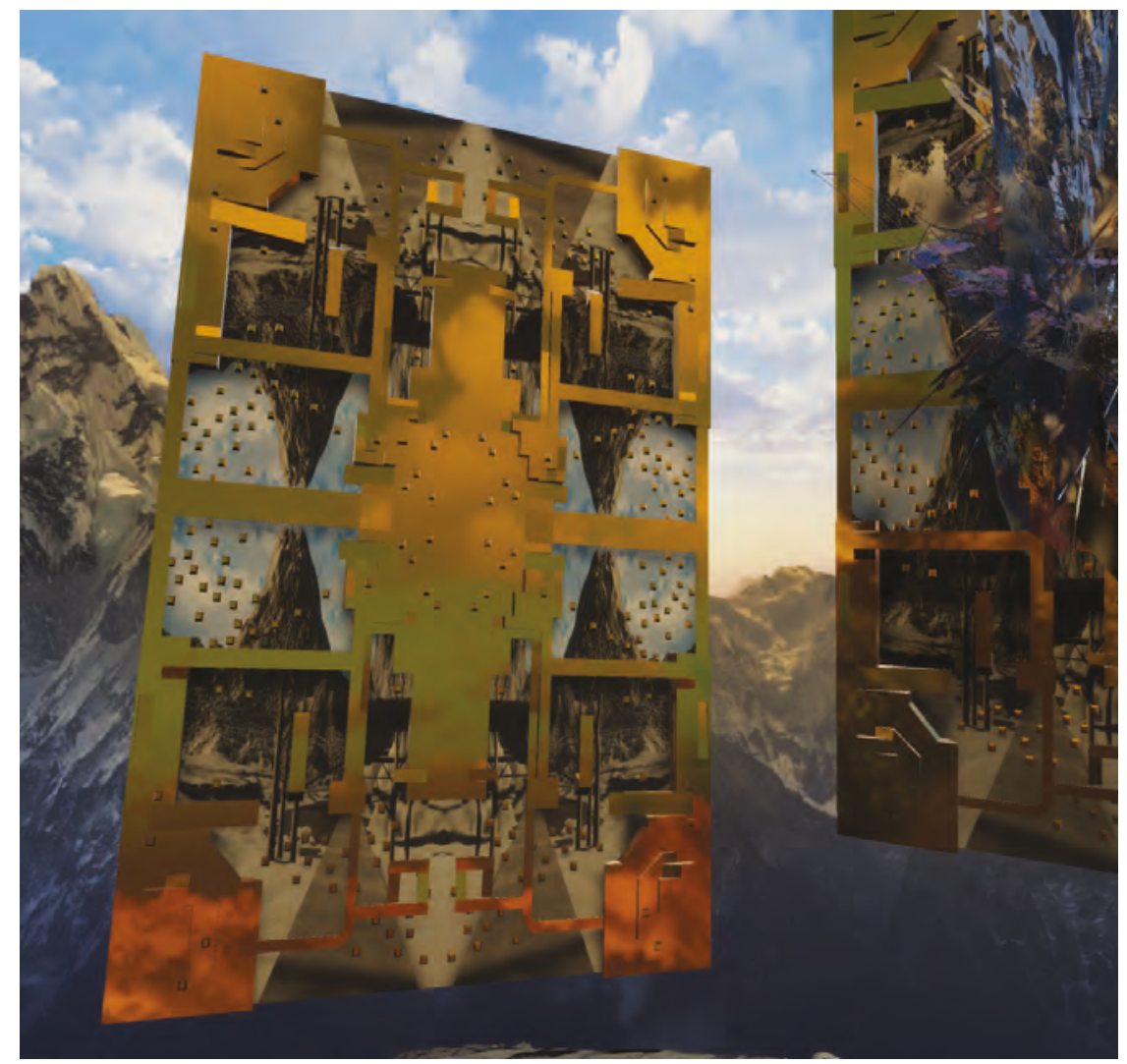

FIG. 71 PERSPECTIVE - THE ORIGINAL MAP FROM LEVEL 2 FLOATS ALONG SIDE THE 2-DIMENSIONAL PLANES FROM LEVEL 1

\section{EXPERIENCE}

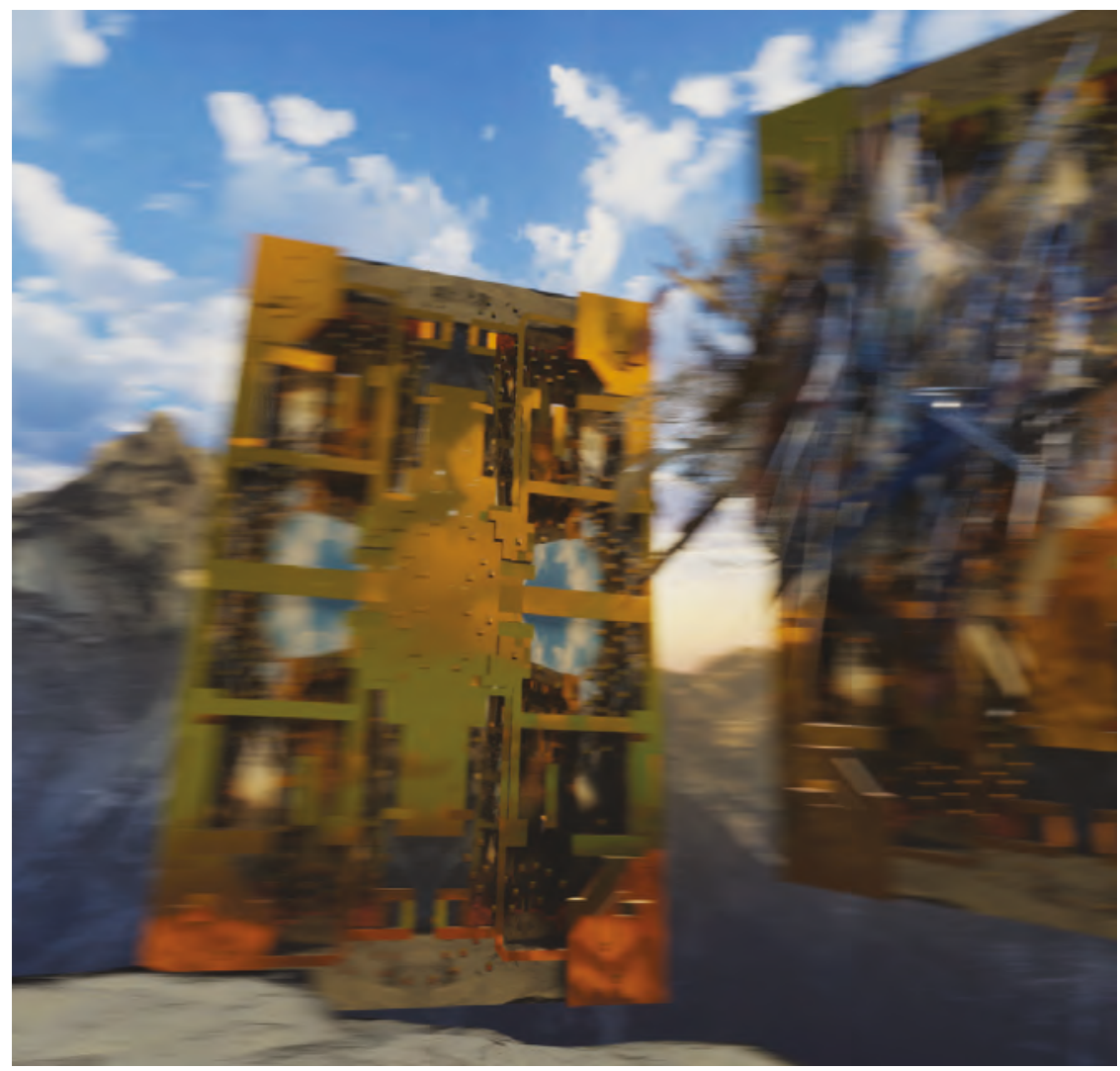

FIG. 72 PERSPECTIVE - THE PLANES ORBIT IN A CIRCULAR PATTERN WHILE SOME OF LEVEL 2'S MAP REFLECTS THE SKY WHILE OTHER AREAS REFLECT THE PLAYERS GAZE 
Level 3 Gameplay Videos

Level 3 Gameplay with Sound Effects

https://www.youtube.com/watch?v=bn509Ce7dWM

Level 3 Early Gameplay

https://www.youtube.com/watch?v=SL-DwwfZuso 


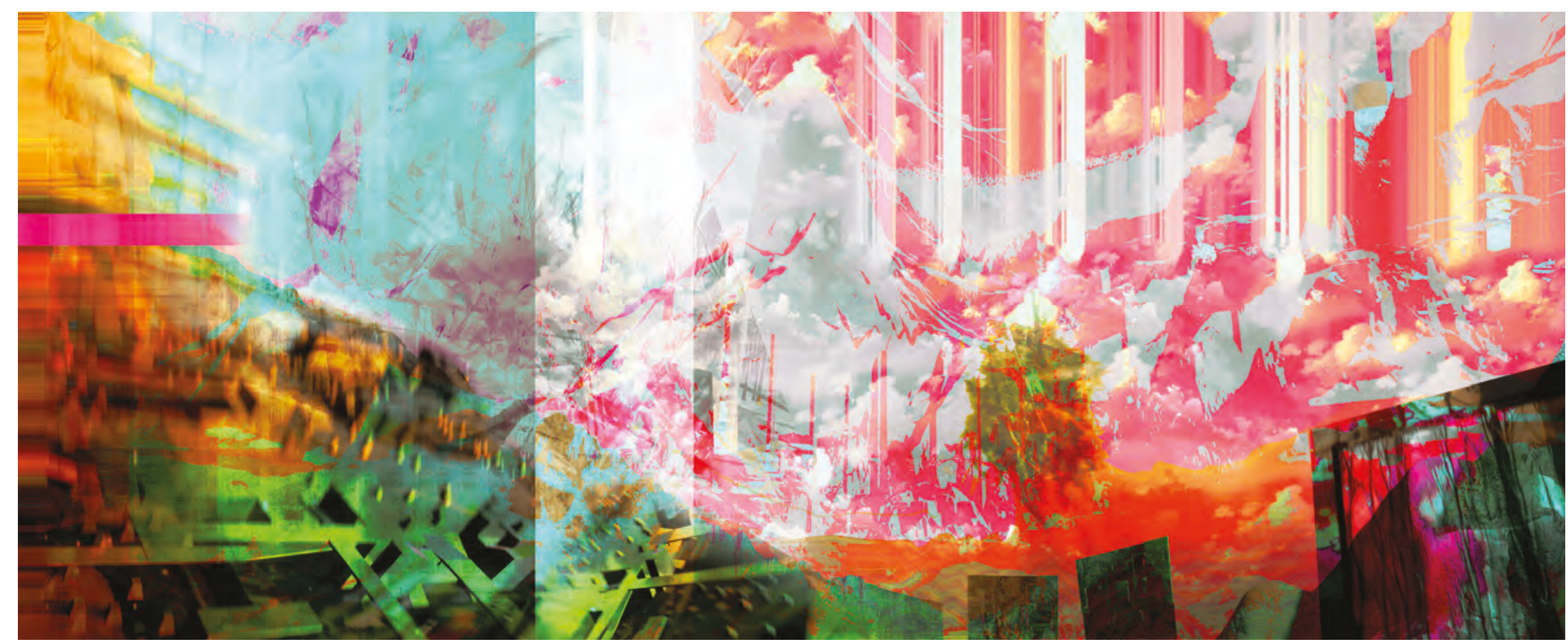

FIG. 73 LEVEL 3 PERSPECTIVE AND GLITCH COLLAGE 
CONCLUSION

This thesis has revealed to me that the game is much closer to the actual experience of architecture, the built work, than the representational experience of architecture, the drawing. At the beginning of the thesis the goal was to design a game, a digital space that could be interacted and played with. Unlike a professional architecture project, the goal was not to have a physical object that could be built in the end. There are far more similarities between game design and architecture than I had thought coming into the project. The structure of the digital environment and the performative nature of the game became the most important elements. The structure of the digital world and the representation became closely associated with the glitch. Austin and Perrin in an essay titled Drawing the Glitch define the glitch. The essay provides two ways to understand the use of the glitch in my work. They cite Gaulon who defines the glitch as "a way of seeing the code behind a document" (Austin and Perin n.d., 16) which becomes my way of representing the written programming work in visual form. Glitches and data bending techniques were used in the construction of the culminative images at shown at the end of each level in this document. The second way to understand the glitch is found near the end of the essay which reads "the notational nature of drawing means that the glitch transforms it form "a work that is yet to be realized" to a work that cannot be realised without a reworking of what the drawing represents." (Austin and Perin n.d., 18) Paramount to my work was an attempt to represent and experience a world that is not physically inhabitable. While traditional techniques of architectural drawing do allow for an easy transition to the digital world the current traditions of drawing may not be capable of communicating the experience of dwelling within these worlds

FIG. 74 PERFORMANCE TRIPTYCH

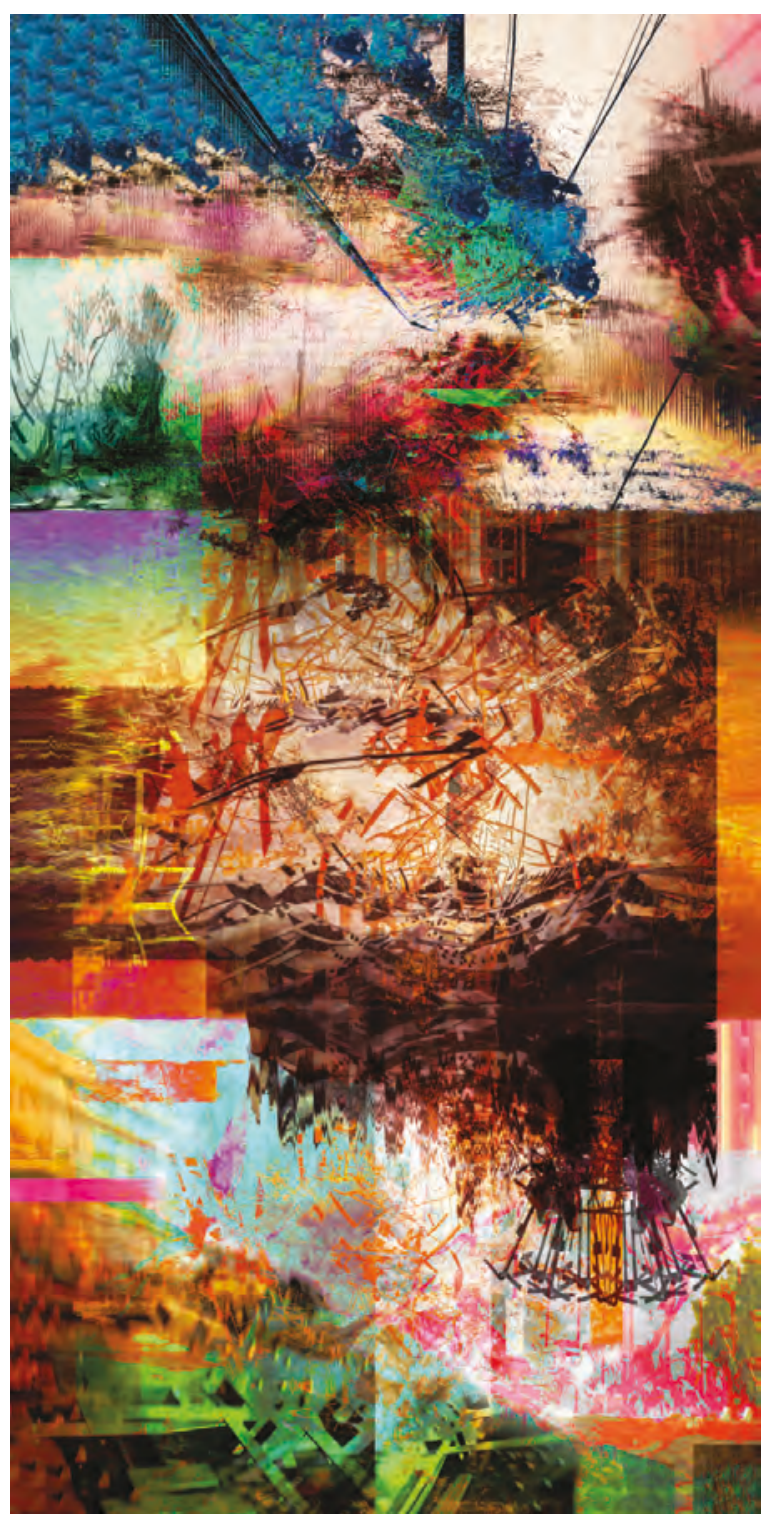




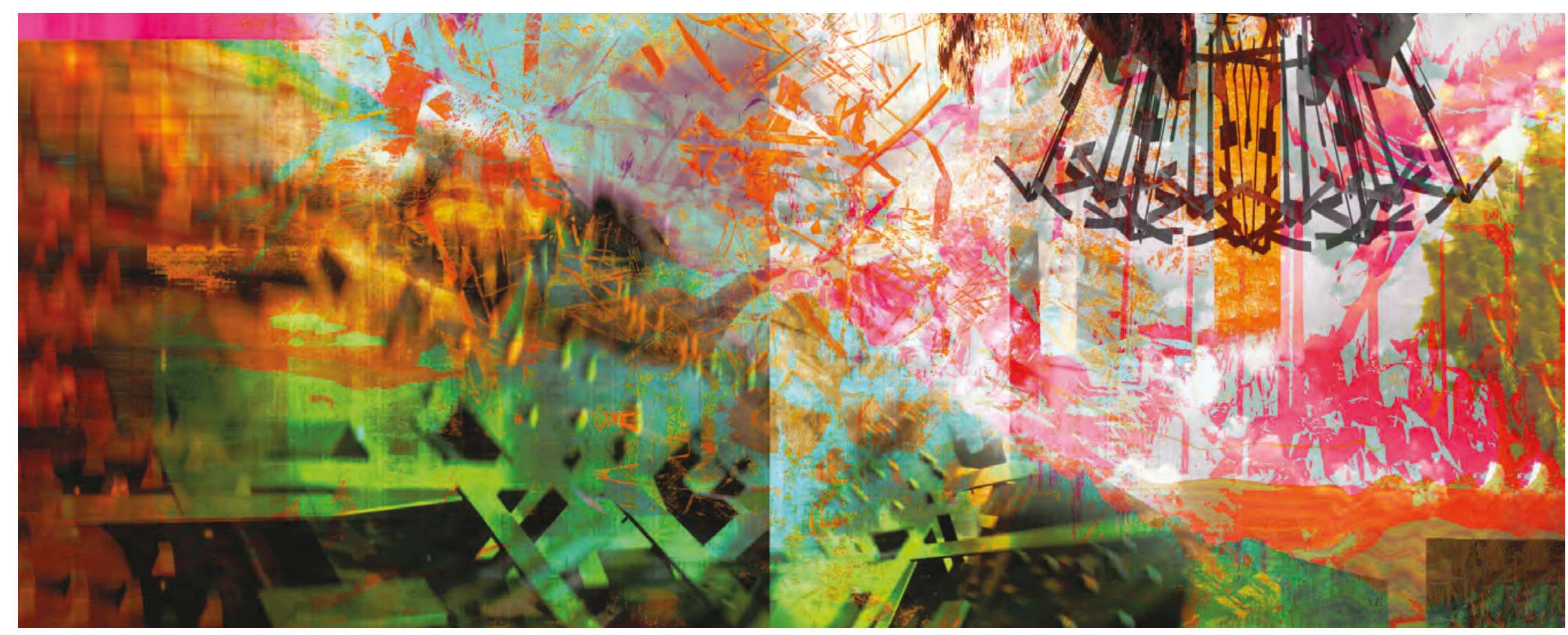

The experience of the playing the games levels proved to be the most difficult element to communicate in this thesis. It is closely associated with the idea of a performance like a play. Researcher in Cultural and Media Studies Johannes Binotto, says in an interview "we play for the sake of playing. There is nothing you take with you from the game once it is over. It is performative medium that consist only of being played." (Binotto 2019) In architecture the idea of representing an experience through drawing has persisted. We might be tempted to look at the drawing versus the game as representation. The drawing has successfully represented experiences of space, but unlike the experience of actual space it is static, a snapshot that will never change. The game provides a way of experiencing a space that is closer to that of actual experience. It is a performance that can not be framed and taken away for another day. I can play the exact same level of my childhood favourites and my interaction with it will be vastly different. Most things will be set up the same, but they will play out differently whether it be from my actions or reactions to code reacting to me differently. With how much closer an interactive digital model is to an actual experience the future of architectural representation could forgo the drawing entirely and focus on the model. This might even lead to a closer connection to the building tradition depending on the advancements in $3 \mathrm{~d}$ modelling technologies to mimic actual world construction. 


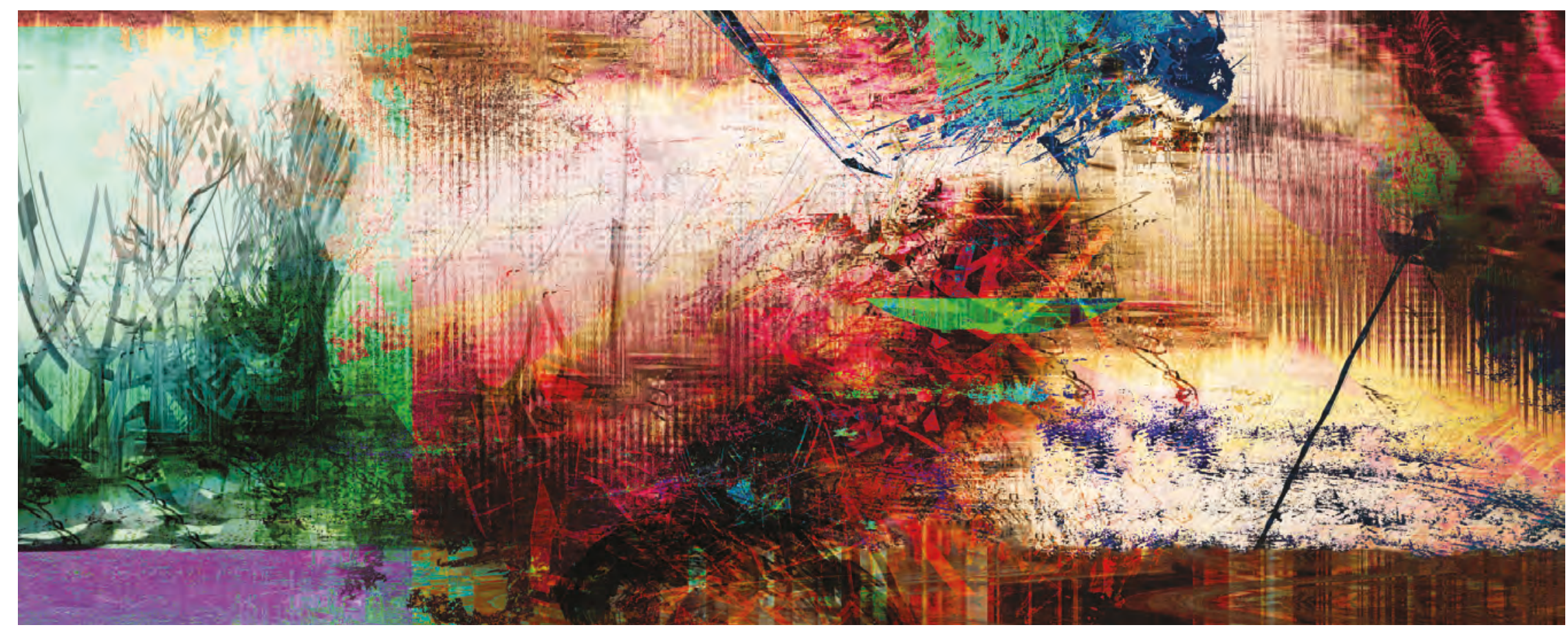

This thesis has explored how we might "feel" or experience virtual realities. The contextual framework established through literary research revealed workflow and conceptual similarities between game design and architectural design. Architectural studies provide a basis to understand 3-dimensional space within video games. Video game studies has revealed the nature of how our bodies interact with the digital environment, directly and through a mediated character. The experience of a virtual reality is dependant on the actions taken within a set of coded rules dictated by the game mechanics. It is in this space that a player can 'play' a game. Play is the result of the combination of writing code to build a virtual environment and defining how the body mechanically interacts within and with this environment. When the character and by extension the player can probe the limits of these rules, sometimes causing glitches, they are able to develop an understanding of the context. Considerations can now be given to the what the experience of a virtual reality might reveal. The tools for understanding and exploring experience in games apply to virtual worlds in architecture as well. Representation can be interacted with and more importantly allowed to evolve along side the experience of a space. When the representation and experience of a context exist in the same reality, and where that experience is close enough to an actual lived experience, we can 'feel' a representation. 


\section{LEVELS DOWNLOAD LINK}

https://drive.google.com/drive/folders/1aXk3vJIEDGILPh-XpV4H3kISdmJQX7M9?usp=sharing 


\section{Term Definitions}

Gamespace- The virtual space where the game objects, camera's, and event systems are instantiated into

Game Object- An item in a game space that can have a script, texture, animation, shader and more applied to it

Scenes- Individual instances of gamespaces

Collider- A bounding box around a gameobject that defines where collisions can occur with that object, an object without a collider can be seen but has not physics interactions

Mesh- A triangulated series of connected points within the gamespace

Mesh Renderer- A mesh renderer allows the mesh to be seen by drawing it in the gamespace based on the material

Displacement Mapping- A height map texture defines deformations of the points of the mesh object

Pixel Displacement- The illusion of depth based on a height map 


\section{Term Definitions Continued}

Vertex displacement- The gamespace transformation of mesh points based on the height map

Material- The image and effects wrapped around a mesh that are rendered in the gamespace

Shader-The sum of all the textures and effects that are applied to a game object

Script- A C\# coding language program using the Unity Engine that runs when the game is started

Prefab- A group of game objects

Procedural Generation- Scripts that run when the game runs in order to create elements based on algorithms such as landscapes, trees, objects, enemies, and structures

Random Generation- A process of generating objects randomly based on a hexadecimal seed

Mouse Input- Mouse movements and button presses 


\section{Term Definitions Continued}

Raycast- "traces lines from the camera position through the cursor position and into the scene, to detect what it hits. If the ray hits a collider, then the Event System dispatches events to the target object" 3.6- Unity Game development Cookbook

Event System - tracks the cursors position using a raycast and allows the engine to detect when it over a game object

Rigidbody- When applied to a game object it allows the Unity Engine to simulate physics on the object

Freelook- The ability to look around using the mouse cursor, or first person look sometimes called mouselook

Mechanics- The systems by which the game is played 


\section{BIBLIOGRAPHY}

Anable, Aubrey. 2018. Playing with Feelings. Minneapolis: University of Minnesota Press.

Austin, Matthew, and Gavin Perin. n.d. "Drawing the Glitch.” In Drawing Futures, by Laura Allen and Luke Caspar Pearson.

Binotto, Johannes, interview by Andri Gerber. 2019. Video/Game (February 1).

Chattopadhyay, Swati. 2012. "Architectural Representations, Changing Technologies, and Conceptual Extensions." Journal of the Society of Architectural Historians (University of California Press) 71 (3): 270-272. www.jstor.org/stable/10.1525/sjah.2012.71.3.270.

Grosz, Elizabeth. 2001. "Cyberspace, Virtuality, and the Real: Some Architectural Reflections." In Architecture from the Outside : Essays on Virtual and Real Space, by Elizabeth Grosz, 74-91. Cambridge: Cambridge, Mass. : MIT Press.

Keogh, Brendan. 2018. A Play of Bodies: How We Perceive Videogames. Cambridge: MIT Press.

Loe, Casey. 2005. Tom Clancy's Splinter cell. Prima's official strategy guide. Roseville, CA: Prima Games.

Lunato, Ben, interview by Paper Guy. 2020. Steam (October 25).

Picon, Antoine. 2003. "Architecture, Science, Technology, and the Virtual Realm." In Architecture and the Sciences: Exchanging Metaphors, by Alessandra Ponte and Antoine Picon, 294-311. New York: New York: Princeton Architectural Press.

Wark, McKenzie. 2007. Gamer Theory. Cambridge, MA: Harvard University Press. 


\section{TUtORIAL BiBLIOGRAPHY}

The 6 Design Patterns Game Devs Need? YouTube, 2020. https://youtu.be/hQE8IQk9ikE.

6 Key Principles for 3D Modeling. YouTube, 2019. https://youtu.be/OVbIOHAI3iY.

Basics of Shader Graph- Unity Tutorial. YouTube, 2018. https://youtu.be/Ar9eln4z6XE.

Best Free Building \& Prop Assets: Unity Asset Store 2019. YouTube, 2019. https://youtu.be/n8RLbydIBzU.

Best Free Nature Assets: Unity Asset Store 2019. YouTube, 2019. https://youtu.be/oUsT7AgzcR8.

BEST MODELING SOFTWARES for Unity 2019! YouTube, 2019. https://youtu.be/xr7xt7pDqL8.

Best Practices for Fast Game Design in Unity- Unite LA. YouTube. Unity, 2018. https://youtu.be/NU29QKag8a0.

Better Data with Scriptable Objects in Unity! (Tutorial). YouTube, 2020. https://youtu.be/PVOVIxNxxeQ. 


\section{Tutorial Bibliography Continued}

Blender 2.9 Beginner Tutorial- Part 1. YouTube, 2020. https://youtu.be/bpvh-9H8S1g.

Blender Tutorial: 20 Essential Selection Tips For Modeling. YouTube, 2018. https://youtu.be/f38jz2qIVZI.

Build Beautiful Terrains with Unity 2019! - New Terrain Tools Package. YouTube, 2019. https://youtu.be/aExdxF4OKBo.

Coding Adventure: Portals. YouTube. Sebastian Lague, 2020. https://youtu.be/cWpFZbjtSQg

Coding Adventure: Portals. YouTube. Sebastian Lague, 2020. https://youtu.be/cWpFZbjtSQg.

Coding Adventure: Procedural Moons and Planets. YouTube. Sebastian Lague, 2020. https://youtu.be/IctXaT9pxA0.

Create a Subway in Blender in 20 Minutes. YouTube, 2018. https://youtu.be/nb6rSMAooDs.

Create Mandelbulb Fractals In Blender Eevee. YouTube, 2018. https://youtu.be/WSQFt1Nruns. 


\section{Tutorial Bibliography Continued}

Create Seamless Tiling Texture Maps Free: Bitmap to Material Tutorial. YouTube, 2017. https://youtu.be/aHOMg0OP260.

Creating a Third Person Camera Using Cinemachine in Unity! (Tutorial). YouTube, 2020. https://youtu.be/537B1kJp9YQ.

Creating Generative Visuals with Complex Systems- Simon Alexander-Adams. YouTube, 2020. https://youtu.be/VBzIPLh-ECg.

The Darkest Corner of Dishonored. YouTube, 2021. https://youtu.be/ANkh3DXMqWo.

Designing a 4D World: The Technology behind Miegakure [Hide\&Reveal]. YouTube, 2016. https://youtu.be/vZp0ETdD37E.

Destruction in Blender for Absolute Beginners. YouTube, 2020. https://youtu.be/ogWQs_7DUOY.

DISPLACEMENT IN UNITY. YouTube, 2019. https://youtu.be/-cb5ooWLSIg.

DISSOLVE Using Unity Shader Graph. YouTube, 2018. https://youtu.be/taMp1g1pBeE. 


\section{Tutorial Bibliography Continued}

EASY Mandelbulb in Blender 2.8 w/ Mandelbulb3D: Voxel Slice Method. YouTube, 2019. https://youtu.be//17_3llav5w.

EVERY Image Effect in Unity Explained- Post Processing v2 Tutorial. YouTube, 2020. https://youtu.be/9tjYz6Ab0oc.

Fast Fractals With Blender. YouTube, 2020. https://youtu.be/CR7jxw94Tcg.

FIRST PERSON MOVEMENT in Unity-FPS Controller. YouTube. Brackeys, 2019. https://youtu.be/_QajrabyTJc.

Flocking with GameObjects and Unity's C\# Job System. YouTube, 2020. https://youtu.be/KJZoSV-JX5I.

FORCE FIELD in Unity- SHADER GRAPH. YouTube, 2019. https://youtu.be/NiOGWZXBg4Y.

Game Architecture Tips- Unity. YouTube. Dapper Dino, 2019. https://youtu.be/pRjTM3pzqDw.

Gaming's Harshest Architecture: NaissanceE and Alienation. YouTube. Jacob Geller, 2019. https://youtu.be/Zkv6rVcKKg8. 


\section{Tutorial Bibliography Continued}

GRASS SWAY in Unity- SHADER GRAPH. YouTube, 2019. https://youtu.be/L_Bzcw9tqTc.

HDRP vs. URP- Which Unity Template Should You Choose? YouTube, 2020. https://youtu.be/5MuA92xUJCA.

How Bevel Profiles Changed Blender Forever. YouTube, 2020. https://youtu.be/D2MwHYxc2uM.

How Do Non-Euclidean Games Work?: Bitwise. YouTube, 2020. https://youtu.be/IFEIUcXCEvl.

How I Improve the Graphics in Unity! (Tutorial). YouTube, 2019. https://youtu.be/jrgN_71s_Go.

How to Create a Low Poly Tree in 45 Seconds. YouTube, 2019. https://youtu.be/IGE-CcjdxN8.

How to Import Blender 2.8 \& 2.9 to Unity? YouTube, 2019. https://youtu.be/NjflKgMepQs.

How to Make 3D Fractals. YouTube, 2018. https://youtu.be/svLzmFuSBhk. 


\section{Tutorial Bibliography Continued}

How to Make Your First Game TODAY!- (Unity 3D). YouTube. Jonas Tyroller, 2018. https://youtu.be/pCBqgREiSUE.

How to Program in C\#- Variables (E02). YouTube. Brackeys, 2020. https://youtu.be/g-9Jp4dmOBo.

How to Randomly Generate Levels (and Islands). YouTube, 2019. https://youtu.be/O9J_Cfl6HzE.

How to Use the Stencil Buffer in Unity. YouTube, 2020. https://youtu.be/-NB2TR8IjE8.

Implementing a Bomb Arrow: Prototype Series. YouTube, 2021. https://youtu.be/Rr Ot9h7igU.

LEARN UNITY- The Most BASIC TUTORIAL I'II Ever Make. YouTube. Imphenzia, 2020. https://youtu.be/pwZpJzpE2IQ.

Learning the Basics of the Unity Raymarching Toolkit Asset. YouTube, 2018. https://youtu.be/QDfqgG8HJDQ.

Let's... NatureManufacture Forest Environment \& R.A.M 2019 HD RP. YouTube, 2019. https://youtu.be/BcomSbnCMSU. 


\section{Tutorial Bibliography Continued}

Making Portals with Shader Graph in Unity! (Tutorial). YouTube, 2019. https://youtu.be/TkzASwVgnj8.

MESH GENERATION in Unity- Basics. YouTube. Brackeys, 2018. https://youtu.be/eJEpeUH1EMg.

NEW INPUT SYSTEM in Unity. YouTube. Brackeys, 2018. https://youtu.be/Pzd8NhcRzVo.

Non-Euclidean 3D Modeling- Hyperbolica Devlog \#5. YouTube, 2021. https://youtu.be/spDA3hPJf6E.

Pathfinding in Unity DOTS! (Insane Speed!!!). YouTube, 2020. https://youtu.be/1bO1FdEThnU.

Photorealistic Desert in Unity HDRP: Castle Valley Collection. YouTube, 2020. https://youtu.be/TxLdL46rfxg.

Photorealistic Materials in Unity! YouTube, 2018. https://youtu.be/_LaVvGlkBDs.

Photorealistic Misty Forest- Speed Level Design: Unity 2020. YouTube, 2020. https://youtu.be/WLbv6bMyloE. 


\section{Tutorial Bibliography Continued}

Practical Procedural Generation for Everyone. YouTube, 2017. https://youtu.be/WumyfLEa6bU.

Procedural Generation For Beginners: Randomize Object Placement. YouTube, 2018. https://youtu.be/tyS7WKf_dtk.

Procedural Landmass Generation (E01: Introduction). YouTube. Sebastian Lague, 2016. https://youtu.be/wbpMiKiSKm8.

PROCEDURAL TERRAIN in Unity!- Mesh Generation. YouTube. Brackeys, 2018. https://youtu.be/64NblGkAabk.

Proximity Decimation Technique in Blender. YouTube, 2018. https://youtu.be/kL3emU5W05w.

Psuedo-Non-Euclidean Geometry in Unity [A Response Video of Sorts]. YouTube, 2019. https://youtu.be/ukdIAcfGKF4

Shader Fundamentals- Normal Mapping. YouTube, 2017. https://youtu.be/6_-NNKc4Irk.

Snowtracks Shader- Unity CG/C\# Tutorial [Part 1- Tesselation Theory]. YouTube, 2018. https://youtu.be/Sr2KoaKN3mU. 


\section{Tutorial Bibliography Continued}

Terrain Tips \& Tricks: Unity 2019-Tutorial. YouTube, 2019. https://youtu.be/bq_PIBWw5ol.

Tissue- Branching Systems in Blender 2.80. YouTube, 2019. https://youtu.be/7Liv--XSaCg.

Top 40 Best Blender Addons. YouTube, 2020. https://youtu.be/VWyjYqKINqo.

Tween Curves Command (2019): Rhino 3D CAD Technique \#21 (2019). YouTube, 2019. https://youtu.be/ga24rJ5N_SU.

Ultimate 10+ Shaders FREE UNITY ASSET. YouTube, 2020. https://youtu.be/wNrXmihzKLU.

Unity 2018- Game VFX- Shatter / Destroy / Explode Objects Tutorial. YouTube, 2018. https://youtu.be/w4aMtKeD2w4.

Unity Level Design Tutorial with the Map Magic. YouTube, 2016. https://youtu.be/BxqaYrRII5A.

Unity Shaders Tutorial: Using the Stencil Buffer. YouTube, 2020. https://youtu.be/AATjjduNUAU. 


\section{Tutorial Bibliography Continued}

Unity Tips and Tricks for Photorealism: Tutorial 2020. YouTube, 2019. https://youtu.be/Rfn9zM8Dfog.

Unity Tutorial Basic: Constructing a Fractal 1. YouTube, 2020. https://youtu.be/JLPilOEEcD8.

Unity Tutorial: Volumetric Lighting. YouTube, 2018. https://youtu.be/zoN3wZz8bsU.

Unity VFX-Ground Cracks: Fissure: Hole Effect Tutorial. YouTube, 2020. https://youtu.be/qiAiVaOHtyE.

Useful Resources for Making Landscapes in Blender! YouTube, 2021. https://youtu.be/Se4e2DQ_D5g.

What Are Events? (C\# Basics). YouTube, 2020. https://youtu.be/OuZrhykVytg. 